\title{
Transmit Power Control in Fixed Cellular Broadband Wireless Systems
}

\author{
By \\ Salem Salamah \\ A thesis submitted to \\ the Faculty of Graduate Studies and Research \\ in partial fulfillment of \\ the requirements of the degree of
}

Master of Engineering

Ottawa-Carleton Institute for Electrical and Computer Engineering

Department of Systems and Computer Engineering

Carleton University

Ottawa, Ontario

Copyright 2000, Salem Salamah 
Acquisitions and Bibliographic Services

395 Wellington Street Ottawa ON K1A ON4 Canada
Bibliothèque nationale du Canada

Acquisitions et services bibliographiques

395. rue Wellington Ottawa ON K1A ON4 Canada
The author has granted a nonexclusive licence allowing the National Library of Canada to reproduce, loan, distribute or sell copies of this thesis in microform, paper or electronic formats.

The author retains ownership of the copyright in this thesis. Neither the thesis nor substantial extracts from it may be printed or otherwise reproduced without the author's permission.
L'auteur a accordé une licence non exclusive permettant à la Bibliothèque nationale du Canada de reproduire, prêter, distribuer ou vendre des copies de cette thèse sous la forme de microfiche/film, de reproduction sur papier ou sur format électronique.

L'auteur conserve la propriété du droit d'auteur qui protège cette thèse. $\mathrm{Ni}$ la thèse ni des extraits substantiels de celle-ci ne doivent être imprimés ou autrement reproduits sans son autorisation.

\section{Canadä}




\section{ABSTRACT}

Broadband wireless access systems such as Local Multipoint Communications Services (LMCS) is aiming to provide multimedia communication services to subscribers in fixed locations via millimeter wave transmissions at $28 \mathrm{GHz}$. In LMCS the total allocated frequency band is reused in each cell/sector through the use of highly directional antennas and polarization reuse in adjacent sectors. Some of the key issues in LMCS systems are the coverage and the cochannel interference. These problems have to be resolved before a successful deployment of such services.

In this thesis, we implement two techniques that are known to combat co-channel interference; these techniques are power control and macrodiversity. The objective of this research is to analyze the system performance of LMCS system using these techniques and under different scenarios. We will provide system designers with the appropriate power control command rate and power control step size. Furthermore, the effect of macrodiversity on system availability is investigated.

A computer simulation program was developed and used to determine the system performance of the LMCS network model. The results of the simulation are obtained and presented for different propagation environment and system parameters. The investigated parameters include the propagation exponent, lognormal deviation, Rician $\mathrm{K}$ factor, correlation factor of fading channel, power control rate and step size. 


\section{ACKNOWLEDGEMENT}

In the beginning, I am grateful and praise worthy to Allah who created me and gave me the strength and courage to fulfil my ambitious.

My special gratitude to my thesis supervisor professor Halim Yanikomeroglu for his exceptional help and guidance throughout this research work. My great respect and appreciation for my thesis co-supervisor, Professor David Falconer who offered me the full extent of his valuable experience and support. Working with both Professors was a honour and a life time experience for me.

My praise to my parents, who encourage and support me without their prayers I couldn't reach this stage. I am indebted to my family who stood behind me with encouragement and prayers.

My appreciation to D. Lemay from the technical support staff in the department of systems and computer engineering who provided great assistance in facilitating my access to the required technical resources for my research.

Finally, my special thanks to my friend Hadee Akhand for his helpful discussion. 


\section{TABLE OF CONTENTS}

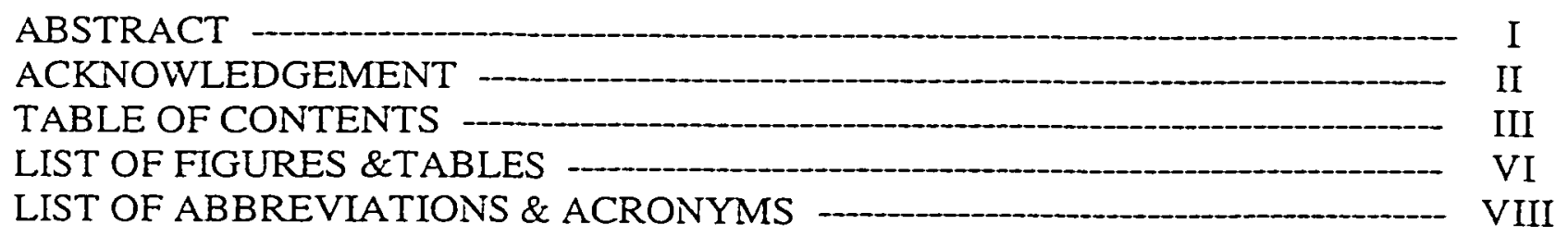

Chapter 1 INTRODUCTION-1 1

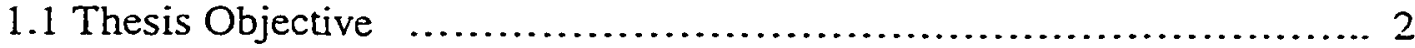

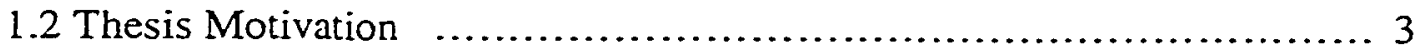

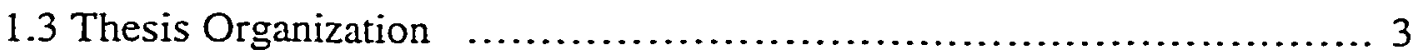

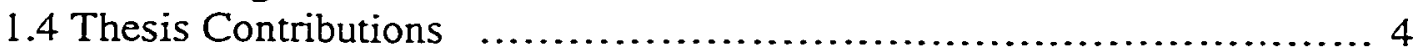

Chapter 2 Local Multipoint Communication System -

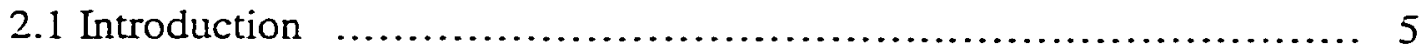

2.2 Applications and Service performance ............................... 7

2.3 Frequency band and Spectrum Allocation $\ldots \ldots \ldots \ldots \ldots \ldots \ldots \ldots \ldots . . . \ldots \ldots$

2.4 Cell architecture.................................................... 10

2.5 Propagation Impairments......................................... 11

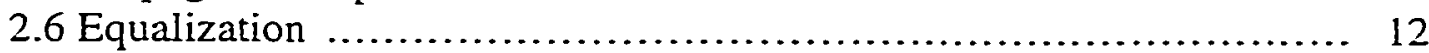

2.7 Error Control Coding............................................. 12

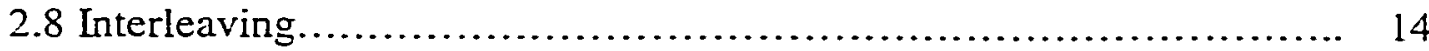

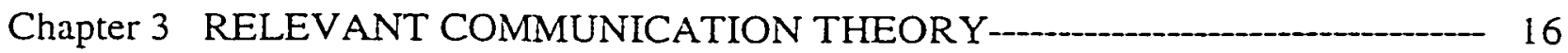

3.1 Propagation in Mobile Communication environment....................... 16

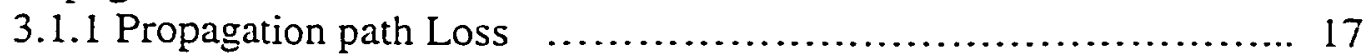

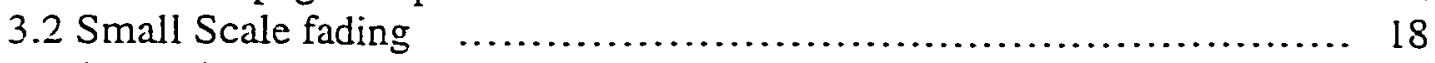

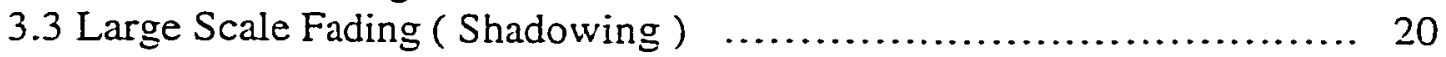

3.4 Frequency Reuse and Interfernce.................................. 21

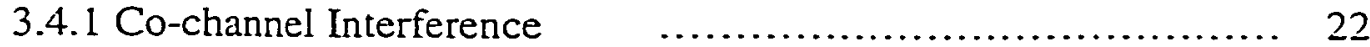

3.4.2 Adjacent Channel Interference................................. 23

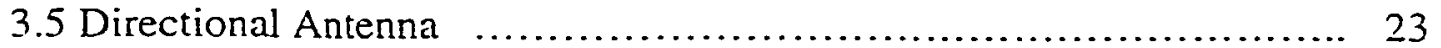

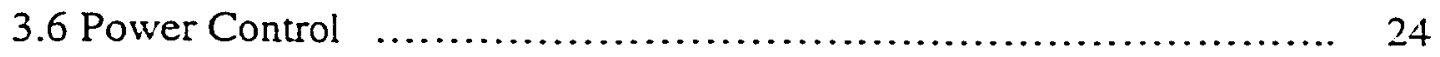

3.6.1 Inner Loop Power Control ................................... 26

3.6.1.1 Open Loop Power Control ............................ 26

3.6.1.2 Closed Loop Power Control .......................... 27

3.6.2 Outer Loop Power Control ................................. 28 


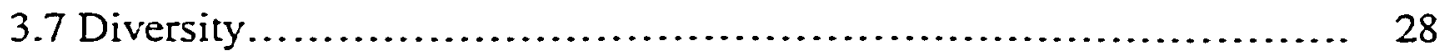

3.7.1 Long Term Fading Counter................................. 29

3.7.2 Short Term Fading Counter .................................... 29

3.8 Outage Probability and System Availability ........................ 30

Chapter 4 SIMULATION MODEL-__- 32

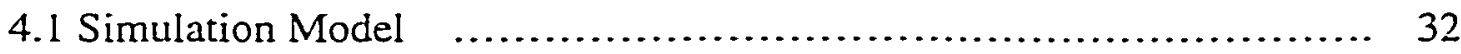

4.1.1 System Model ............................................ 32

4.1.2 Propagation Model ........................................... 34

4.1.2.1 Path Loss model ......................................... 34

4.1.2.2 Large Scale Fading (Shadowing) .......................... 35

4.1.2.3 Small Scale Fading ....................................... 35

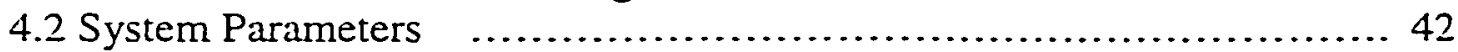

4.2.1 Directional Antenna .......................................... 45

4.2.2 Dynamic Range ........................................... 43

4.2.3 Thermal Noise .......................................... 43

4.3 Binary SINR Based Power Control.................................. 44

4.4 Multi-step SINR-based Power Control............................. 47

4.5 Simulation Algorithm............................................. 49

4.5.1 Description of Simulation Software .......................... 51

4.5.2 Collected Data .............................................. 51

4.6 Issues to be simulated........................................... 53

Chapter 5 SLMULATION RESULTS -_._._. 55

5.1 Binary SNR based Power Control................................. 55

5.1.1 Power control command rate and step size ...................... 56

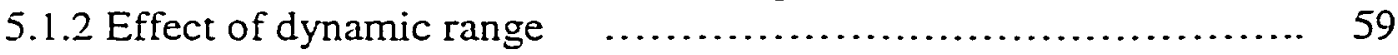

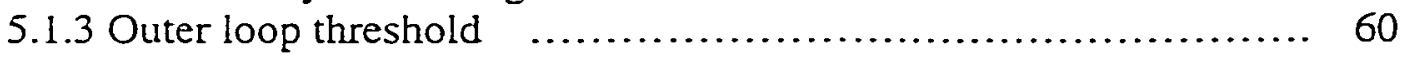

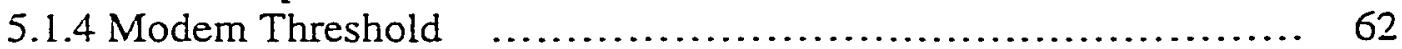

5.2 Effects of Propagation Environment............................... 63

5.2.1 The effect of propagation exponent ............................ 64

5.2.2 Standard deviation of shadowing .............................. 66

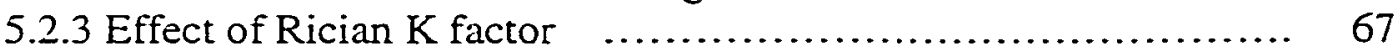

5.2.4 Effect of Correlation factor .................................... 69

5.3 Multi step SNR-based Power Control .......................... 72

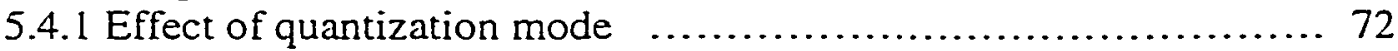

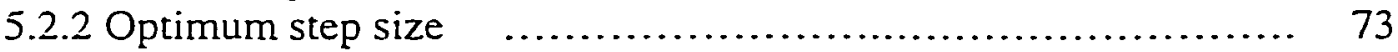

5.4 Binary SINR based vs. Multi-step power control $\ldots \ldots \ldots \ldots \ldots \ldots . . . \ldots . . . .64$

5.5 Effect of Macrodiversity $\quad$........................................ 76

5.6 Autocorrelation and power spectral density $\ldots \ldots \ldots \ldots \ldots \ldots \ldots \ldots . . \ldots \ldots \ldots$

Chapter 6 CONCLUSIONS - 80

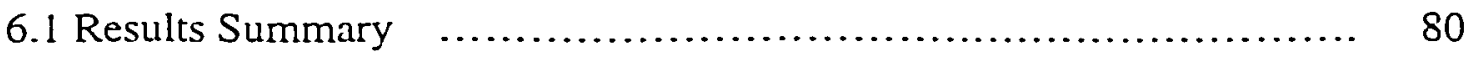




\section{LIST OF TABLES \& FIGURES}

Figure 2.1 LMCS/LMDS band allocation

9

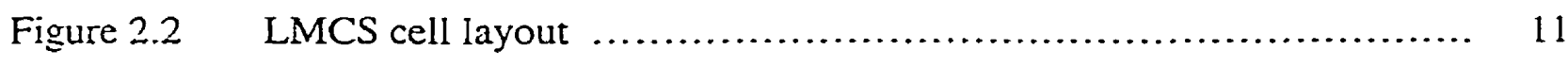

Figure 2.3 Probability of bit error for Reed Solomon and convolotional codes 13-14

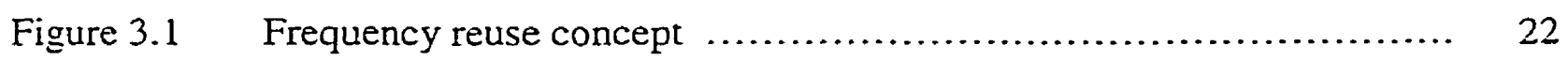

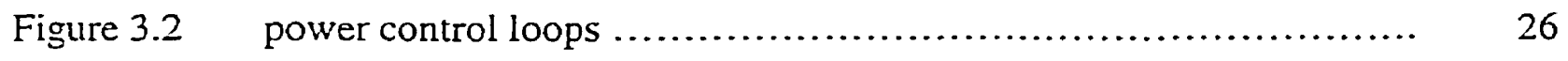

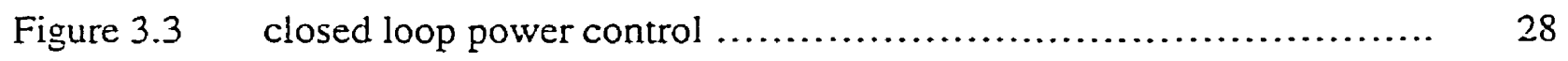

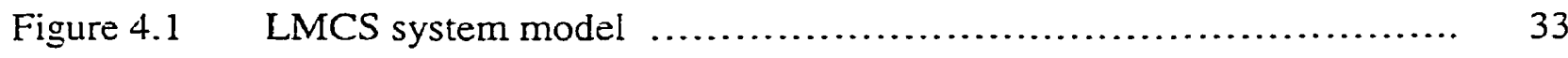

Figure 4.2 Time correlation filter for Rician fading $\ldots \ldots \ldots \ldots \ldots \ldots \ldots \ldots \ldots \ldots \ldots \ldots \ldots \ldots \ldots$

Figure 4.3 Envelope power for a Rician channel with different correlation factors $39-41$

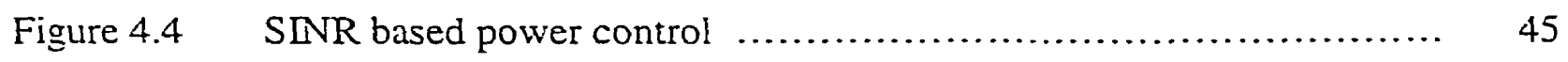

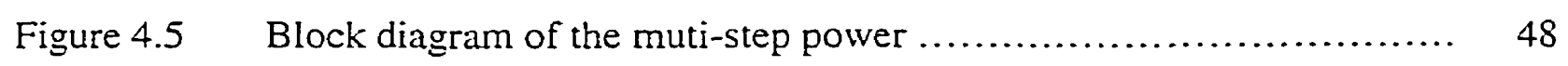

Figure $4.6 \quad$ Flow diagram for the simulation program $\ldots \ldots \ldots \ldots \ldots \ldots \ldots \ldots \ldots \ldots \ldots \ldots \ldots \ldots \ldots$

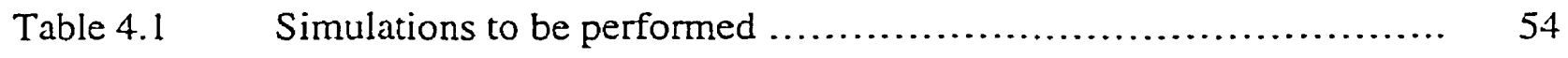


Figure 5.1 System availability as function of power control rate and step size

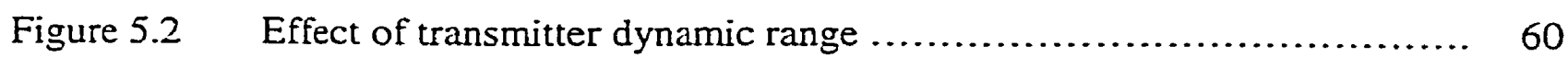

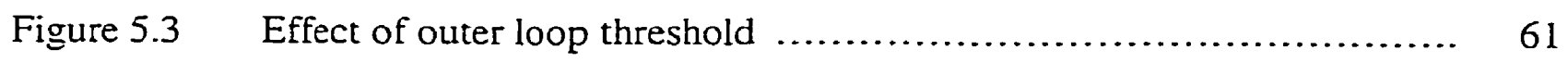

Figure $5.4 \quad$ System availability as function of modem threshold $\ldots \ldots \ldots \ldots \ldots \ldots \ldots . . . \ldots 3$

Figure 5.5 System availability versus propagation exponent of desired subscriber ... 65

Figure 5.6 System availability vs. propagation exponent of interferer subscriber ... 66

Figure $5.7 \quad$ System availability vs. deviation of lognormal shadowing $\ldots \ldots \ldots \ldots \ldots .67$

Figure $5.8 \quad$ The effect of desired subscriber Rician K factor ......................... 69

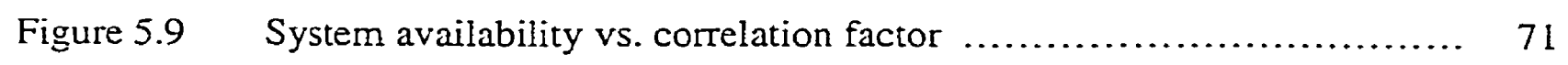

Figure 5.10 System availability versus the mode of multi-step power control ........ 73

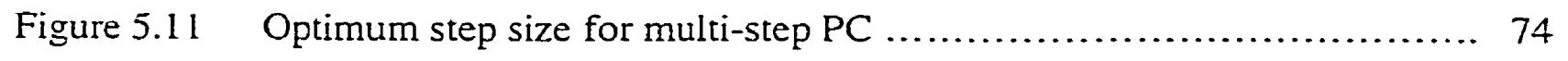

Figure 5.12 Binary SINR based versus multi-step PC $\ldots \ldots \ldots \ldots \ldots \ldots \ldots \ldots \ldots \ldots . \ldots \ldots$

Figure $5.13 \quad$ The effect of macrodiversity on system availability $\ldots \ldots \ldots \ldots \ldots \ldots \ldots . \ldots 7$

Figure 5.14 Auto correlation function for Rician channel $\ldots \ldots \ldots \ldots \ldots \ldots \ldots \ldots \ldots \ldots .78$

Figure $5.15 \quad$ Power spectral density of the fading signal $\ldots \ldots \ldots \ldots \ldots \ldots \ldots \ldots \ldots \ldots \ldots$ 


\section{LIST OF ACRONYMS}

\begin{tabular}{|c|c|}
\hline LMCS & Local Multipoint Communication Systems \\
\hline LMIDS & Local Multipoint Distribution Services. \\
\hline LOS & Line of sight. \\
\hline FCC & Federal Communications Commission. \\
\hline BWA & Broadband wireless access \\
\hline FTTH & Fiber-to-the-home. \\
\hline HFC & Hyprid-fiber-coax \\
\hline PONs & Passive Optical Networks \\
\hline ADSL & Asynchronous digital subscriber loop \\
\hline ATMI & Asynchronous transfer mode \\
\hline MSS & Mobile Satellite Service \\
\hline BS & Base Station \\
\hline BER & Bit Error Rate. \\
\hline FER & Frame Error Rate. \\
\hline FEC & Forward error correction \\
\hline CDF & Cumulative Distribution Function. \\
\hline pdf & Probability density function \\
\hline CDMA & Code Division Multiple Access. \\
\hline TDMA & Time Division Multiple Access. \\
\hline FDMA & Frequency Division Multiple Access. \\
\hline SINR & Signal-to-Interference plus Noise Ratio. \\
\hline SIR & Signal-to-Interference Ratio. \\
\hline SNR & Signal to noise ratio \\
\hline $\mathbf{C} / \mathbf{I}$ & Carrier to interference ratio \\
\hline AWGN & Additive White Gaussian Noise. \\
\hline ISI & Inter-symbol Interference \\
\hline $\mathbf{L}$ & Path Loss. \\
\hline DFE & Decision feedback equalization \\
\hline $\mathbf{R S}$ & Reed Solomem Code \\
\hline QAMI & Quadrature Amplitude Modulation. \\
\hline QPSK & Quadrature Phase Shift Keying \\
\hline dB & decibel \\
\hline $\mathbf{H z}$ & Hertz \\
\hline $\mathbf{M H z}$ & Mega Hertz \\
\hline GHZ & Giga Hertz \\
\hline Msps & Mega symbol per second \\
\hline
\end{tabular}




\section{LIST OF SYMBOLS}

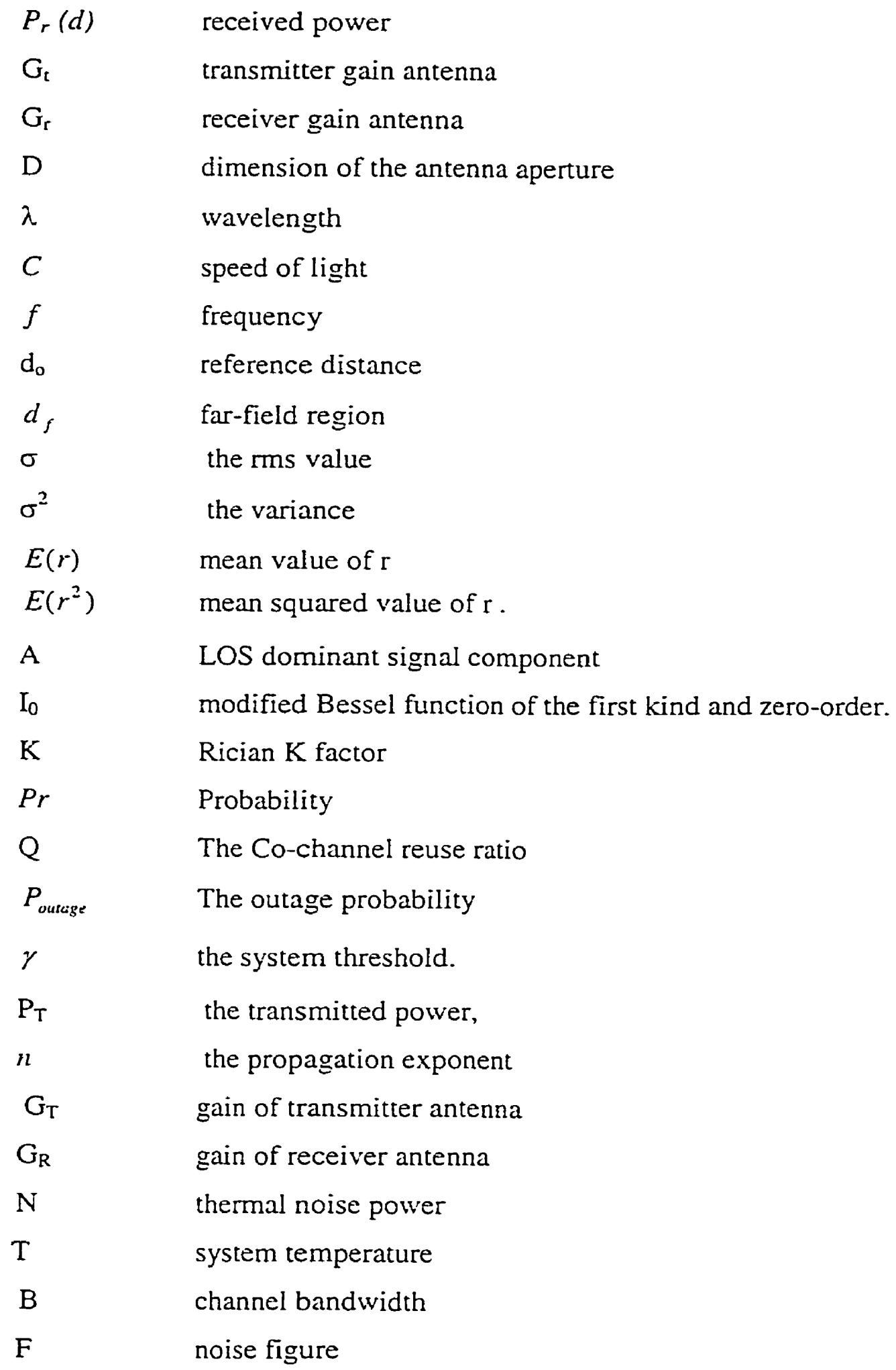




$\begin{array}{ll}S & \text { desired signal } \\ \mathrm{I} & \text { interference } \\ \alpha & \text { correlation factor } \\ \mathrm{T}_{\mathrm{p}} & \text { waiting period } \\ \Delta & \text { power control step size } \\ \gamma(\mathrm{th} & \text { power control threshold } \\ \mathrm{C}(\mathrm{e}) & \text { power control command. } \\ c m d & \text { power control command for multistep PC } \\ \mathrm{PC} & \text { power control }\end{array}$




\section{Chapter 1 \\ INTRODUCTION}

The increasing demand for multimedia type, high bit rate services motivated researchers to develop broadband wireless access technologies. The delivered services are broadcasting TV, video on demand, high speed internet access etc...

Broadband access systems will serve both residential and business customers in fixed networks.

One of the promising broadband access technologies is the local multipoint distribution services (LMDS) or local multipoint communication systems (LMCS), which has been introduced to deliver a wide variety of broadband services. This wireless technology is competing against wireline broadband systems such as Fiber-to-the-home (FTTH), hybrid-fiber-coax (HFC), and Asynchronous Digital Subscriber Loop (ADSL) on copper wires [6].

The main advantages of LMDS/LMCS over wireline technologies are easy operation and deployment, flexibility in on-demand capacity allocation and potential support for a broad spectrum of applications, allowing for future development, in addition to, its lower initial infrastructure and gradual increment in subscriber cost. The proposed system is a bi-directional broadband wireless system to fixed networks at millimeter wave frequencies.

The problem that developers and operators are facing is the efficient utilization of the spectrum $(1.3 \mathrm{GHz})$ for LMCS. It is due to the nature of challenging propagation environment at such high frequency. Hence, the coverage is an important issue, which needs to be resolved before taking full advantage of this application. 


\subsection{Thesis Objective}

The main objective of this thesis is to study the effect of transmit power control on the performance of fixed broadband wireless systems in the frequency range of $28 \mathrm{GHz}$, which is known as LMCS/LMDS.

Outage performance and coverage of LMCS system are to be investigated and analyzed under different conditions and scenarios with regard to a number of system parameters of interest.

These parameters include propagation-environment-related parameters such as propagation exponent, standard deviation of lognormal shadowing, Rician fading $\mathrm{K}$ factor, and time correlation factor for fading channel. The other set of parameters related to the implementation of power control schemes is power control command rate, power control step size, transmitted power dynamic range and outer loop threshold. In this study, we will analyze the influence that each parameter has on the overall performance and availability of the service for fixed subscribers in the LMCS system.

The objectives of this research can be summarized as follows:

1. To study the effect of propagation environment parameters on power control effectiveness.

2. To examine the influence of power control parameters on system performance.

3. Exploit the macrodiversity technique to mitigate cochannel interference.

The thesis objective is achieved by simulating LMCS system model with parameter values that make the model as realistic as possible. In simulation, only uplink direction (fixed subscriber to base) is considered.

Propagation measurements conducted to date indicate that coverage in suburban areas depending primarily on the cell size, antenna heights, and the density of trees and 
buildings in the area. At carrier frequencies of $28 \mathrm{GHz}$ or higher, the wavelength is in the range of $1 \mathrm{~cm}$; for this reason buildings and trees which obstruct the line of sight (LOS) typically result in very high signal attenuation. Therefore, one of the main issues that have to be resolved is the coverage.

\subsection{Thesis Motivation}

Power control and macrodiversity have shown promising results in a previous study, where a coarse power control scheme is employed [9]. The proposed research aims to address the coverage problem and to manage co-channel interference with the implementation of power control and macro diversity techniques.

In this thesis, we will employ a finer closed loop power control scheme with macro diversity to mitigate co-channel interference and multipath fading. The target is to enhance the outage probability and service availability for fixed subscribers, thus increasing the service coverage area.

\subsection{Thesis Organization}

This document is organized into six chapters. Chapter 1 describes the thesis objective, motivation and contributions. Chapter 2 provides a brief review of LMCS systems. Its applications and system design issues such as error control coding, equalization and interleaving.

In Chapter 3 we will review some of the communication theory and the propagation characteristics of the channel. Directional antennas are also described. A survey for power control theory and diversity techniques is discussed as well in this chapter.

Chapter 4 introduces the LMCS system model along with the assumptions that were used in this study. 
Chapter 5 gives a summary of the simulation results.

Finally, in chapter 6 a conclusion of our discussion and recommendation for further studies are present.

\subsection{Thesis Contributions}

This thesis contains a performance analysis of LMCS system employing power control and macrodiversity in different cond itions with regard to some system parameters of special interest as mentioned earlier.

A simulation tool was developed to ewaluate the performance of the LMCS model under consideration. As far as it can be dettermined based on a review of the literature, this research has some general contributioras that can be summarized as follows:

1. Analyzing the performance of the LMCS system with respect to the environment parameters such as the propagation exponent, Rician $\mathrm{K}$ factor, standard deviation of log normal shadowing.

2. Studying the effect of the power control parameters on the system performance for LMCS network. These parameters are the power command update rate, power control step size, transmitted power dynamic range and outer loop threshold..

3. Observing the impact of macrodiversity technique on system performance. 


\section{Chapter 2}

\section{LOCAL MULTIPOINT COMMUNICATION SYSTEM}

This chapter describes the local multipoint communication systems LMCS architecture. LMCS system is the fixed broadband wireless access in the range of $28 \mathrm{GHz}$. It is very attractive because it provides broadband services to residential and business customers. Although there are promising applications, there exist some issues to be solved before utilizing the potential of this band. These issues include coverage and co-channel interference. These problems arise because of the hostile propagation environment.

\subsection{Introduction}

Recently local Multipoint Communication System (LMCS) or local Multipoint Distribution System (LMDS) has been proposed in Canada and the United states for wireless access to broadband services.

LMCS is a broadband wireless access technology that is intended to provide broadband services to fixed subscribers in small cells. LMCS systems are designed to have cellular layout. They attempt to completely reuse the frequency band in each cell through the use of highly directional subscriber antenna and polarization reuse in adjacent cells, so that the interference from co-channel subscribers in adjacent cells can be significantly reduced [6].

The acronym LMDS or LMCS is derived from the following:

- $L$ (local) denotes that propagation characteristics of signal in this frequency range limit the potential coverage area of a single cell site; ongoing field trials conducted in metropolitan centers place the range of an LMDS transmitter at up to 5 miles. 
- $M$ (multipoint) indicates that signals are transmitted in a point-to-multipoint or broadcast method; the wireless return path, from subscriber to the base station, is a point-to-point transmission.

- $D$ (distribution) or $C$ (communication) refers to the distribution of signals, which may consist of simultaneous voice, data, Internet and video traffic.

- $S$ (service) implies the subscriber nature of the relationship between the operator and the customer; the service offered through an LMDS network is entirely dependent on the operator's choice of business.

The advantages of the LMCS over the competitive access technologies such as Hybrid Fiber Coax (HFC) and Passive Optical Networks (PONs) are as follows

- Low entry and deployment cost

- Ease and speed of deployment: deployment of cable and fiber systems is difficult in certain areas where installing in-ground infrastructure is undesirable. LMCS can provide similar access bandwidths and a two way capability without trenching streets and yards.

- Faster realization of revenues as a result of rapid deployment.

- Quick response to growing market.

- Bandwidth on demand: Any or all the bandwidth is available to all subscribers within the range of the hub.

LMDS provides a wireless alternative to fiber, coax, and asynchronous/very high-rate digital subscriber line (ADSL/VDSL) and offers a high capacity locally compared with other radio solutions like interactive satellite systems [31].

Despite the above mentioned advantages, there are few disadvantages as well which can be stated as follows:

- Because of the nature of frequency reuse there is always the possibility of co-channel interference 
- In the frequency range of $28 \mathrm{GHz}$ and above the wavelength is of the order of millimeter. This poses the problem of coverage. With such a small size of wavelength, tree buildings, terrain and even rain drops cause a high attenuation.

It has been demonstrated by field measurements that signal attenuation due to obstructing trees is the most serious propagation impairment [13]. The system performance is limited by the ability of the system in providing sufficient signal strength over radio links. Propagation characteristics of millimeter waves require that transmission should be line of-sight; this means small coverage cells. Consequently we have a larger number of cells for a given area and therefore an increased number of base stations and distribution infrastructure.

\subsection{Applications and Service Performance}

LMCS and LMDS are wireless terrestrial bi-directional communication systems that are intended to provide broadband services to home and business subscribers within covered service areas. The targeted services range from one-way video distribution and voice telephony to fully interactive switched broadband multimedia applications. Some of these applications are as follows:

- Video on demand application

- Broadband Internet access

- Interactive multimedia

- Home office

- Distance education

- Voice and Video Telephony

- Entertainment TV

- Interactive video games

- Home shopping

The required service performance for LMCS can be summarized as follows [7]: 
- Call set up $<10 \mathrm{sec}$.

- Isochronous cell loss rate $<10^{-3}$ asynchronous cell loss rate $<10^{-4}$.

- Maximum delay $<50 \mathrm{~ms}$.

\subsection{Frequency Band and Spectrum Allocation}

Regulatory agencies such as the U.S. Federal Communications Commission (FCC) are authorizing point-to-multipoint radio systems to operate over a block of spectrum and throughout a large geographical area.

FCC has proposed two separate licenses, one license for a bandwidth of $1150 \mathrm{MHz}$, which includes the spectrum from 27.5 to $28.35 \mathrm{GHz}, 29.1$ to $29.25 \mathrm{GHz}$ and 31.075 to 31.225 GHz. This spectrum is referred as Block $A$. The second license, referred as Block $\mathrm{B}$, includes the spectrum from 31 to $31.075 \mathrm{GHz}$ and 31.225 to $31.3 \mathrm{GHz}$, a total of 150 $\mathrm{MHz}[5]$.

Industry Canada granted two blocks of $500 \mathrm{MHz}$ in the 27.35 to $28.35 \mathrm{GHz}$ ranges. Additional spectrum from 25.35 to $27.35 \mathrm{GHz}$ has been designated for LMCS future use. Frequency bands in US is shown in Fig.2.1

The segment beginning at $29.1 \mathrm{GHz}$ is shared with the mobile satellite service (MSS) and has several restrictions. The restrictions include: [32]

1. Subscriber stations may not transmit.

2. LMDS hub antennas are restricted as to upper elevation gain.

3. The aggregate radiated power per square kilometer is restricted.

4. In some cases coordination is required with MSS earth stations.

These restrictions limit the distance between hub and subscriber sites and increase system design and administration cost. 
Licensing and deployment in Europe now indicate that there will be systems in different frequency bands from $24 \mathrm{GHz}$ up to $43.5 \mathrm{GHz}$. The frequency band $24.5-26.6 \mathrm{GHz}$ with sub-bands of $56 \mathrm{MHz}$ has been opened for point-to-multipoint applications in many European countries. These bands may then be used for LMDS.

\section{LMDS Band Allocation (Local Multipoint Distribution Service) \\ $28 \& 31 \mathrm{GHz}$ Band Plan}

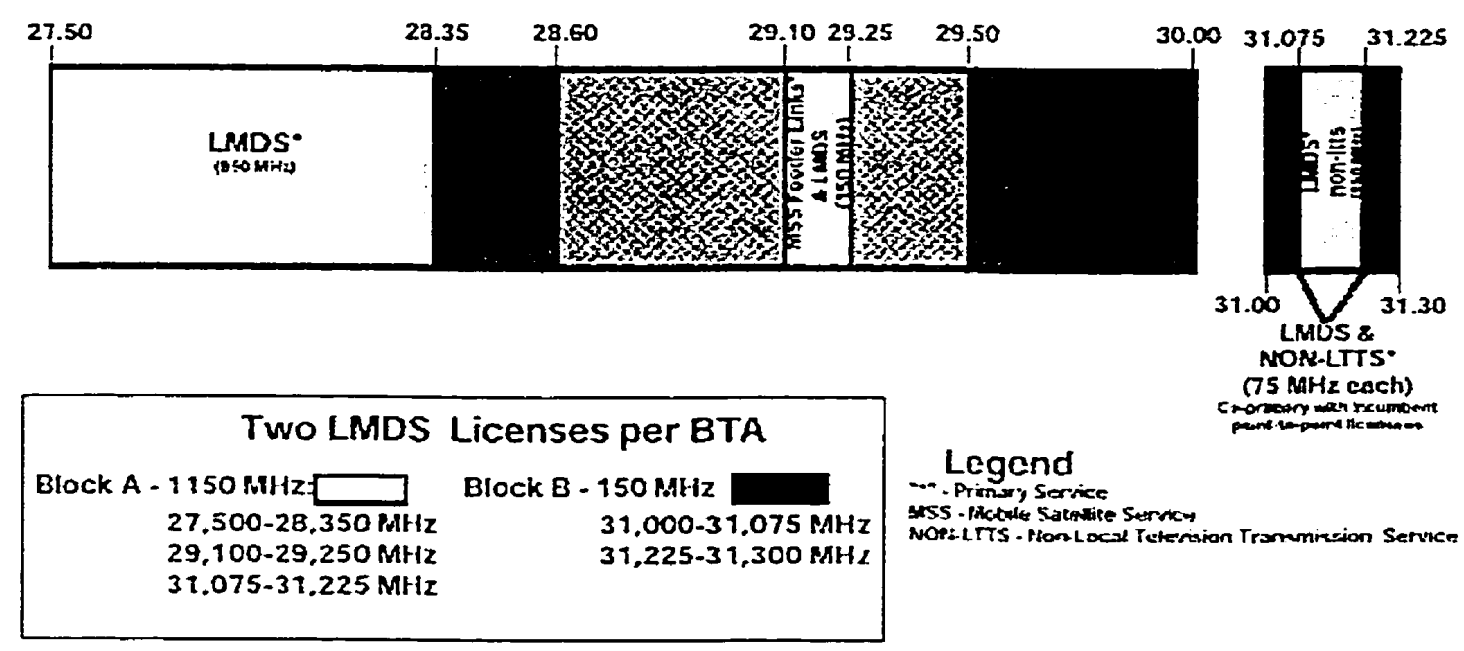

Swarce: Federa: Cormmurikatun: Courmistan

Figure 2.1 LMCS/LMDS band Allocation in USA 


\subsection{Cell Architecture}

The LMCS system is designed to have a cellular architecture and attempts to reuse the total allocated frequency in each sector. This essentially means that frequency will be reused 4 times in each cell. The transmitter site should be on top of a tall building or on a high pole overlooking the service area. A typical configuration is a four-sector cell site using 90-degree beamwidth antennas to provide service to the subscribers. Each of these sectorized antennas can support the full bandwidth of the allocated spectrum. The isolation between adjacent sectors can be maximized through the use of antenna polarization as shown in Fig 2.2.

LMDS has a point-to-multipoint downlink and a point-to-point uplink. In order to provide good coverage, fixed subscribers should have LOS path with the hub. In many locations, this may not be the case due to blockage from buildings and trees. Low power repeaters and reflectors can be used to fill in the areas where there is no available LOS path between the hub and the fixed subscriber. The repeater would amplify and redirect the signal in the direction of the coverage hole. The use of repeaters to extend coverage was studied in [12] and provides an improvement of $6 \%$ coverage with macrodiversity.

The maximum cell size for the service area is related to the desired system availability obtained from the link budget. Cell size can vary due to the type of antenna, its height, and signal loss. Operation in the millimeter range imposes some restrictions. Precipitation effects lead to severe attenuation and limit the reliable range of operation to $3-5 \mathrm{~km}$ depending on the climatic zone and the frequency of operation. 


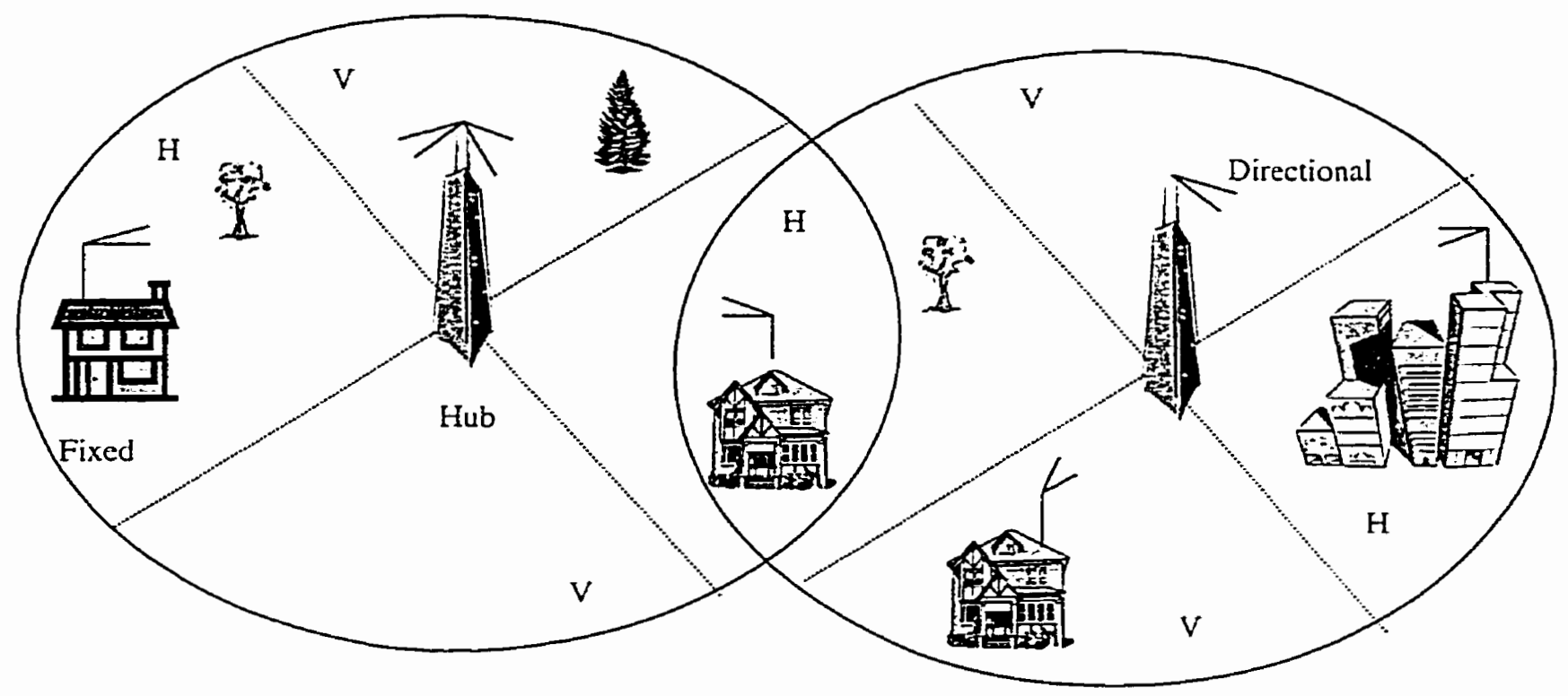

V: Vertical polarization

$\mathrm{H}$ : Horizontal polarization

Figure 2.2 LMCS Cell Layout

\subsection{Propagation Impairments}

The propagation environment at millimeter wave frequencies is one of the major challenges in delivering LMCS services to fixed subscribers. At such high frequencies the signal is attenuated by the obstacles in the radio path between the subscriber and the hub, such as buildings, trees and vehicles. Measurement study for LMCS at $28 \mathrm{GHz}$, in Ottawa shows that foliage cause signal attenuation of more than $20 \mathrm{~dB}$ in some locations. Rain and snow could cause more signal loss [11]. This makes it necessary to provide a line-of-sight path from the hub to subscriber for maintaining sufficient signal strength. In [30] it was shown that even when line-of-sight paths are available, excess loss due to rain attenuation must be accounted for in the link budget. The requirement of line-of-sight for reliable communication at these frequencies is presenting a big challenge for system designers who try to maximize coverage at minimum cost.

Significant difficulty is expected in providing service to residential subscribers, due to a lack of coverage, which, in this situation, results from insufficient relieved power. This 
coverage problem is brought by the use of mm-wave frequencies in combination with the assumption that a subscriber will typically be equipped with a small antenna, mounted on or near the subscriber roof level, which is typically below the level of obstructing trees.

Coverage studies for LMCS system indicate that coverage in suburban areas can vary from nearly $100 \%$ down to $50 \%$ or less, depending primarily on cell size, antenna heights, and the density of trees and buildings in the coverage area.

\subsection{Equalization}

Intersymbol interference (ISI) caused by multipath in bandlimited (frequency selective) time dispersive channels distorts the transmitted signal, causing bit errors at the receiver. ISI has been recognized as the major obstacle to high speed data transmissions over radio channels. Equalization compensates for intersymbol interference [18].

Decision feedback equalization (DFE) has been evaluated in [10] for various data rates based on the multipath spread present in the measured impulse response data from residential area in Ottawa.

The study showed that for data rates of 10 mega symbols per second (Msps) using QPSK modulation and with narrow beamwidth directional antenna at the subscriber, it is possible to avoid equalizer techniques. However, an equalizer is needed with 3 taps for forward filter and I tap for the feedback filter for data rates of $25 \mathrm{Msps}$. The equalization requirements for 50 Msps are 4 or 5 forward filter taps and 1 tap for feedback filter.

\subsection{Error Control Coding}

Error control coding techniques rely on the systematic addition of redundant symbols to the transmitted information to facilitate two basic objectives at the receiver, which are error detection and error correction. The redundant bits lower the raw data rate through the channel. Hence, the spectral efficiency is reduced. 
The study in [11] shows the Reed Solomon code and the convolutional code performance based on the temporal variation of the narrow band LMCS channel studies. The parameters for RS codes are length of code word $n=255$ symbols, packet length $=53$ bytes ( 1 ATM cell) and error correction capability $t=0$ to 10 . The probability of symbol error as function of link margin for QPSK modulation is shown in Fig. 2.3(a).

Convolutional coding is very popular because of its simplicity in terms of hardware implementation. For a constraint length of 7 and code rate $=1 / 2$, the probability of bit error for different link margin is shown in Fig 2.3(b)

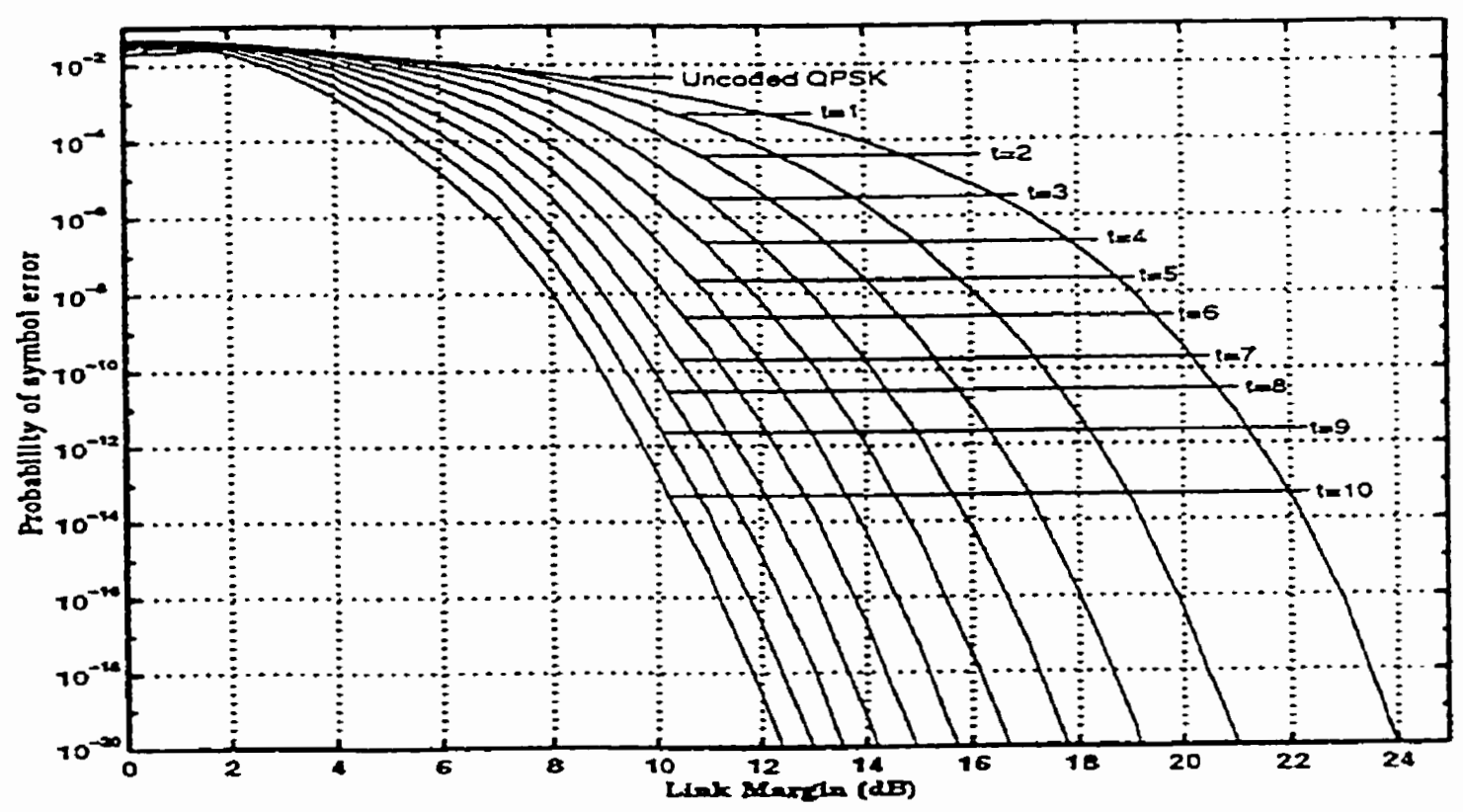

Figure 2.3 (a) [11] 


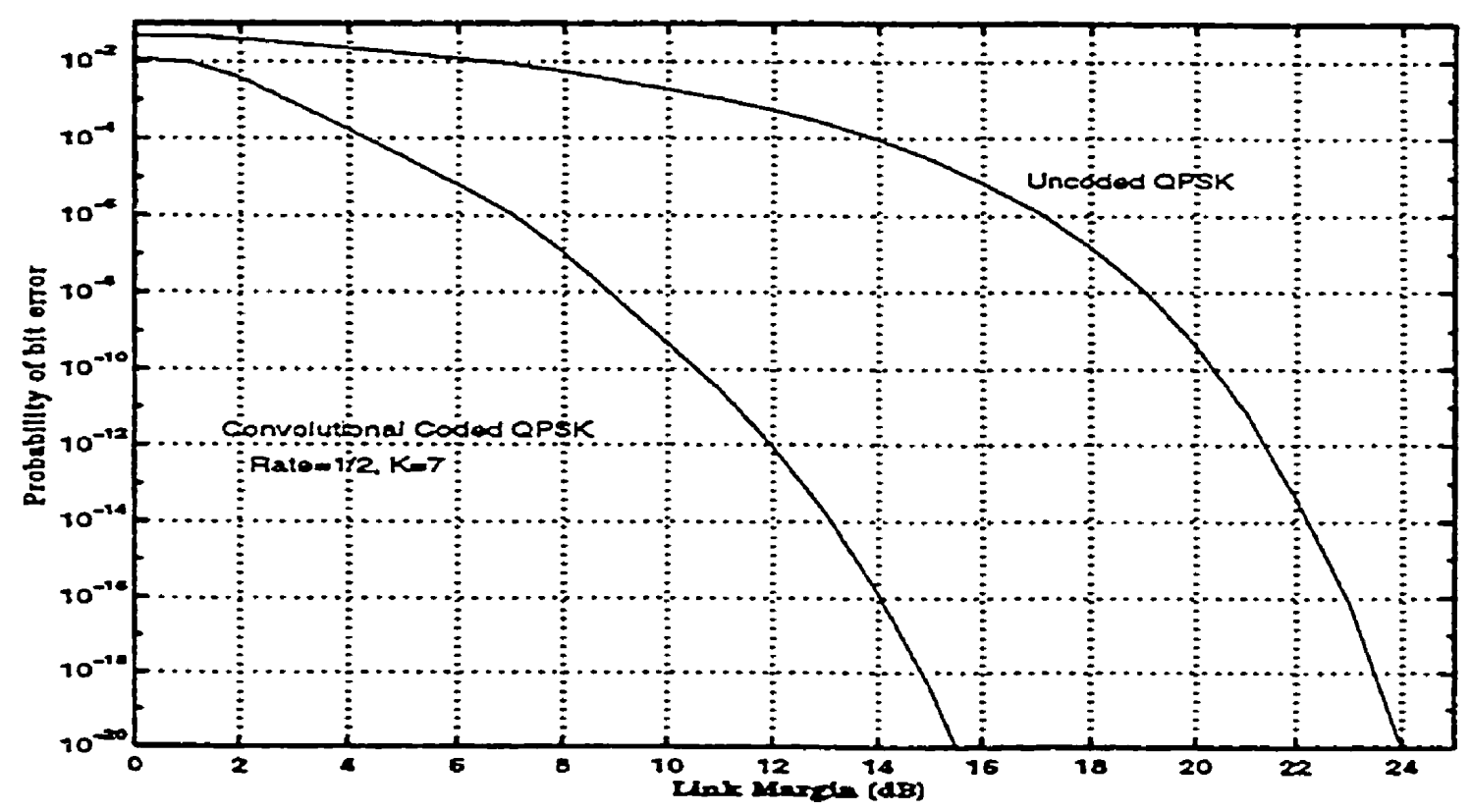

Figure $2.3(b)[11]$

\subsection{Interleaving}

Interleaving is a form of time diversity that is employed to disperse bursts of errors in time. A sequence of data symbols is interleaved before transmission over a bursty channel. If errors occur during transmission, restoring the original sequence to its original ordering has the effect of spreading the error over time. By spreading the data symbols over time, it is possible to use channel coding which protects the data symbols from corruption by channel.

The interleaving techniques can be divided into two categories:

- Block Interleaving

- Convolutional Interleaving

The interleaver performance depends on memory required for data storage and the delay in interleaving and deinterleaving, which should be kept as small as possible. The 
performance of both interleavers was evaluated in [11] using the following parameters with RS codes.

1. Interleaving length $=\frac{3000}{t}$ where $t$ is the error correcting capability $(t=2)$.

2. Data rate $=40 \mathrm{Mb} / \mathrm{s}$.

The delay with convolutional interleaver is $40 \mathrm{msec}$ and using block interleaver is 80 mseconds. Efficiency can be defined as the ratio of the length of the smallest burst of errors that can cause the errors correcting capability of the code to be exceeded to the number of memory element used in the interleaver. An efficiency of $14.2 \%$ for block interleaver and $64 \%$ for convolutional interleaver has been shown in [11]. 


\section{Chapter 3}

\section{RELEVANT COMMUNICATION THEORY}

In this chapter we will discuss the propagation and multipath characteristics of the radio channel such as path loss, fast fading, and shadowing. The concepts of frequency reuse and directional antennas will also be discussed. Power control and diversity that are used to combat cochannel interference and multipath fading will be discussed as well.

\subsection{Propagation in a Mobile Communication Environment}

In a mobile radio environment the propagation phenomena can be characterized using different propagation models. These models can be divided into two groups, namely theoretical and empirical models.

Modeling the radio channel has been one of the most difficult parts of mobile communication systems design. Thus the stochastic behavior of the mobile radio signal may be described by means of statistical distributions. Three distributions are closely related to the mobile radio channel statistics: lognormal, Rayleigh and Rician. The lognormal distribution describes the envelope of the received signal power shadowed by obstructions such as buildings and hills. The Rayleigh distribution describes the envelope of the received signal resulting from multipath propagation. The Rician distribution considers the envelope of the received signal with multipath propagation plus a line-ofsight component. Propagation models are required to quantify two variables:

1 - Average signal strength at any distance from the transmitter.

2-Signal variability which characterizes the fading nature of the channel. 
Fixed broadband wireless systems such as LMCS/LMDS operating at mm-wave frequencies requires a LOS between the hub and the fixed subscriber. Residential subscribers that are fixed in location are highly affected by signal attenuation due to foliage movement obstructing the link especially in windy environment. The distance path loss and shadowing do not change in BWA. But multipath fading is existed in the channel due to foliage and rain attenuation.

\subsubsection{Propagation Path Loss}

Path Loss can be defined as the difference between the transmitted power and the received power. An exact estimate of the path loss in mobile communications is not available. There are many path loss models but we will discuss only the free space path loss model. In the free space model, the ratio between the received power $P_{r}$ and the transmitted power $P_{t}$ is given by the Friis free-space transmission formula as follows [18]

$$
\frac{P_{r}(d)}{P_{r}}=\frac{G_{t} G_{r} \lambda 2}{(4 \pi d)^{2}}
$$

where $d$ is the distance, $G_{t}$ and $G_{r}$ are the transmitter and receiver gains respectively, and $\lambda$ is the wavelength.

Accordingly, the path loss (in decibels) is

$$
L(d B)=-10 \log G_{t}-10 \log G_{r}+20 \log (4 \pi d)-20 \log (\lambda)
$$

Assuming unity antenna gains $G_{t}$ and $G_{r}$, the path loss in $d B$ is given by

$$
L(d B)=20 \log (4 \pi d)-20 \log (c / f)
$$

Where $f$ is the frequency in hertz, $d$ is the distance in $\mathrm{m}$ and $c$ is the speed of light $\left(3 \times 10^{8}\right.$ $\mathrm{rn} / \mathrm{s})$. 
A large-scale propagation model use a close-in distance $d_{b}$, as a known receiver power reference point. The received power at any distance $d\left(d>d_{o}\right)$, may be related to the received power at distance $d_{0}$. Thus received power can be given for free space model by

$$
P_{r}(d)=P_{r}\left(d_{o}\right) \times\left(\frac{d_{o}}{d}\right)^{2} \quad d>d_{o}
$$

The reference distance $d_{o}$ must be chosen such that it lies in the far-field region (i.e. $d_{o}>$ $\left.d_{i}\right)$.

$d_{f}=\frac{2 D^{2}}{\lambda}$ where $D$ is the largest dimension of the antenna aperture.

\subsection{Small Scale Fading}

Small Scale fading refers to the fluctuation of the amplitude of the signal over a short period of time or travel distance. The received signal is usually a superposition of two or more reflected signals. These signals can be called multipath signals, which combine at the receiver antenna and can cause the received signal to distort or fade. The effects of the small scale fading or simply fading can be summarized in the following:

a- Rapid changes of signal amplitude over a small distance or short period of time.

b- Random frequency modulation caused by the Doppler shift.

c- Time dispersion by the multi-path delays.

Different channel conditions produce different types of small-scale fading. The type of fading depends on the following factors : 
- Speed of the mobile: the relative motion between the mobile and the base station results in random frequency modulation due to different doppler shift on different multipath components.

- Speed of surrounding objects: the movement of objects in radio channel induces a time varying doppler shift on multipath component. Their effect dominates the smallscale fading if they have a higher speed compared to the mobile speed.

- Transmission bandwidth of the signal: if the transmitted signal bandwidth is greater than the channel coherence bandwidth, the received signal suffers multipath distortion (ISI)

\section{A. Rayleigh Fading}

It is well known that the envelope of the sum of two quadrature Gaussian noise signals obeys a Rayleigh distribution. The Rayleigh distribution has the following probability density function (pdf) [18]

$$
P(\mathrm{r})=\frac{r}{\sigma^{2}} \exp \left(-\frac{r^{2}}{2 \sigma^{2}}\right) \quad 0 \leq \mathrm{r} \leq \infty
$$

Where $\sigma$ is the rms value of the received signal power before envelope detection, $\sigma^{2}$ is the variance of the received signal, and $\mathrm{r}^{2}$ is the instantaneous power. Rayleigh fading usually applies to scenario where there is no LOS path between the transmitter and the receiver. The corresponding cumulative distribution function $(C D F)$ is given by

$$
P(\mathrm{R})=\operatorname{Pr}(\mathrm{r} \leq \mathrm{R})=\int_{0}^{R} P(r) d r .=1-\exp \left(-\frac{R^{2}}{2 \sigma^{2}}\right)
$$

The mean value of $\mathrm{r}, E(r)=\sqrt{\frac{\pi}{2}} \sigma$, the mean squared value of $\mathrm{r} E\left(r^{2}\right)=2 \sigma^{2}$ and the variance of $r=\left(2-\frac{\pi}{2}\right) \sigma^{2}$ 


\section{B. Rician Fading}

The Rayleigh fading holds only in the case where there is no LOS path. However, when there is a dominant signal component such as line of sight propagation path; the smallscale fading envelope is Rician. As the dominant signal component becomes weaker, the composite signal envelope becomes Rayleigh fading. The Rician distribution is given by

$P(r)=\frac{r}{\sigma^{2}} \exp \left(-\frac{r^{2}+A^{2}}{2 \sigma^{2}}\right) \quad I_{0}\left(\frac{A r}{\sigma^{2}}\right) \quad$ for $A \geq 0, r \geq 0$

Where A denotes the LOS dominant signal component and $\mathrm{I}_{0}$ is the modified Bessel function of the first kind and zero-order. The Rician distribution is often described in terms of the $\mathrm{K}$ factor ratio, which is defined as the ratio between the dominant signal component and the scattered power. It is given by

$$
\begin{aligned}
& K=\frac{A^{2}}{2 \sigma^{2}} \text { or, in terms of } \mathrm{dB} \\
& K=10 \log \left(\frac{A^{2}}{2 \sigma^{2}}\right)
\end{aligned}
$$

It is obvious that when A goes to zero (i.e. the dominant path signal component fades away) the $\mathrm{K}$ will go to zero and the Rician distribution degenerates to the Rayleigh distribution.

\subsection{Large Scale Fading (Shadowing)}

Large scale fading is so called because it characterizes signal strength over a large variations in $T-R$ separation distance. It also describes the variation in the mean signal strength due to the diffraction of the signal caused by the obstacles in the physical channel. Thus, it is also referred to as shadowing. 
As opposed to the rapidly changing small scale fading, shadowing is more or less a local change in the average signal strength because it has a much slower variation around its mean value. Shadowing was found to have a log-normal distribution, which implies that, the measured signal level in $\mathrm{dB}$, at specific $\mathrm{T}-\mathrm{R}$ separation has a normal distribution about a distance dependent mean $m_{r}$ [18]. The pdf of the log normal distribution is

$$
P(r)=\frac{1}{\sqrt{2 \pi \sigma^{2} r}} \exp -\frac{\left.\left[r-m_{r}\right)\right]^{2}}{2 \sigma^{2} r}
$$

where $\sigma_{\mathrm{r}}$ is the standard deviation of the random variable $\mathrm{r}$ (signal level) in $\mathrm{dB}$. The probability of the received signal level exceeding some threshold $r_{0}$ is given by the following

$$
\operatorname{Pr}\left(\mathrm{r}>\mathrm{r}_{\mathrm{o}}\right)=\operatorname{Pr}\left(\frac{r(d)-m_{r}}{\sigma_{r}}>\frac{r_{o}-m_{r}}{\sigma_{r}}\right)=\mathrm{Q}\left(\frac{r_{o}-m_{r}}{\sigma_{r}}\right)
$$

where

$$
\mathrm{Q}\left(\mathrm{z}_{\mathrm{o}}\right)=\frac{1}{\sqrt{2 \pi}} \int_{\mathbf{z}_{0}}^{\infty} \exp \left(-\frac{r^{2}}{2}\right) d r
$$

\subsection{Frequency Reuse and Interference}

Frequency reuse refers to the use of radio channels on the same carrier frequency to cover different areas that are separated from one another by sufficient distances. The service area, can be divided into cells in a regular fashion, therefore, cells can be represented by a regular polygon. The Hexagon was found to serve a given coverage area with the fewest base stations; therefore it can be an ideal choice.

Each cell is assigned a group of radio channels, which is different from those of the neighboring cells. 


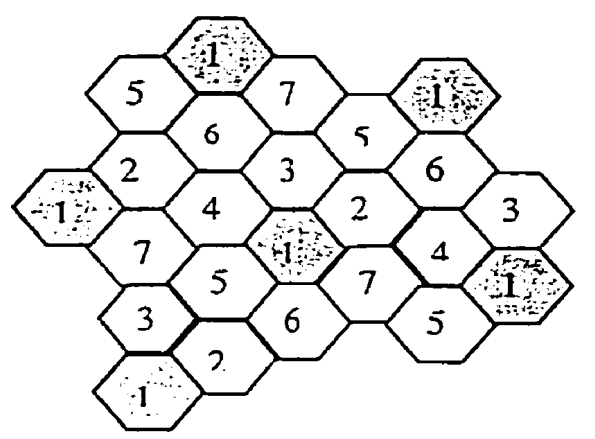

Fig 3.1 Frequency Reuse Concept When $\mathrm{N}=7$.

The number of cells per cluster $\mathrm{N}$ is a parameter of major interest, since in a practical system this number determines how many different channel sets must be formed out of the total allocated spectrum [27]. The frequency reuse factor is defined as $1 / N$.

Interference is one of the major limiting factors in the performance of cellular radio systems. The two major Interference problems in mobile communication systems can be mainly classified as co-channel or adjacent channel.

\subsubsection{Co-channel Interference}

Co-channel interference is a complication that arises in mobile systems because channels are used simultaneously in as many cells as possible, with the minimum acceptable distance, in order to increase the frequency reuse efficiency. This type of interference plays a significant role in determining the capacity of the cellular system. Unlike thermal noise, which can be overcome by increasing the transmitted power, increasing the transmitted power cannot combat co-channel interference. This is because increasing the subscriber transmit power will increase the interference to other co-channel cells.

Reducing the co-channel interference by increasing the co-channel reuse ratio results is a smaller number of cells per cluster, i.e. smaller capacity. A trade-off must be made between co-channel interference and system capacity. 


\subsubsection{Adjacent Channel Interference}

Adjacent channel interference occurrence is basically due to equipment limitations such as receiver bandwidth and filtering. A better control of the adjacent channel interference is possible with the careful filtering and channel assignments by avoiding assigning channels that are all adjacent in frequency to the same cell.

In LMCS systems, each cell attempts to reuse the total allocated frequency band of the system, which makes the co-channel interference significantly high. In our model, we've assumed a well-filtered receiver. Hence, the adjacent channel interference can be ignored.

\subsection{Directional Antennas}

The Directional Antenna is capable of transmitting, or receiving, radio signals with greater power in certain directions and lesser power in other directions. It is also called narrow-beam antenna when its radiated energy is confined to a small solid angle and thus has a narrow-beam main lobe. The gain of the antenna is inversely proportional to the beamwidth; thus a highly directional antenna could have quite high antenna gain in its main lobe.

For LMCS systems which work in the vicinity of $28 \mathrm{GHz}$, it is quite difficult to design the transceiver to provide high transmitting power. By using a highly directional antenna, the transmitted power can be concentrated by the high antenna gain, which is a result of the narrow beamwidth of the directional antenna. The study in [9] finds that highly directional antennas at the subscriber in fixed location can provide large improvements in system performance and could support system design of frequency reuse of one in LMCS systems. A highly directional antenna with small sidelobe can null out interference outside of the mainlobe and therefore improve system performance. 


\subsection{Power Control}

The high demand for wireless communication services shows the need for technology to further increase the capacity of cellular communication systems. Increasing the capacity can be done in many ways, one of them is to reuse the frequency band available for the system in the best manner. The capacity of the system is maximized if the transmitter's power is controlled so that its signal arrives at the base station with minimum required signal-to-interference ratio. If the received signal at the cell site from a subscriber is low, the bit error rate will be too high for that user. On the other hand, if the received signal from a subscriber is too high, the performance for that user will be acceptable, but interference to other users will be increased, which will lower their performance.

Power control schemes must operate rapidly enough to track the changes in path loss, which arises due to mobile movement. If the mobile is moving closer to the base station it will have higher received power at the base from another mobile near the cell border. The closer mobile will capture the base station and this is known as near/far problem.

Power control schemes can be divided based on measurement criteria into the following categories:

1. Signal based power control is based on the original idea of power control to keep the power of all signals received at the base station from all transmitters as nearly as possible equal so as to mitigate the effect of near/far problem. This process is done in a certain step size for each power control command.

2. Signal-to-interference (SIR) based power control relies on the fact that SIR received by base station from all subscribers should be the same.

3. Power balancing power control scheme equalizes the subscriber's signal power to the same level at the base station by adjusting the power by the amount required to achieve the required threshold in one step.

4. SIR balancing power control was proposed to adjust the transmitted power of all mobiles in order to achieve a certain achievable SIR. This algorithm should find 
exactly the transmitted power that each subscriber should transmit with in order to satisfy the required SIR level by the receiver.

Another factor that affects the signal is rapid change in signal level due to mobile movement or scatterers i.e. multipath fading. When the mobile is hidden behind an obstacle like a building it receives a low signal level. If the mobile passes the comer of the building and has a better line-of-sight to the base station it will have a better signal and the mobile should immediately reduce its transmitted power in order to avoid unnecessary interference to other mobiles. Therefore power control must track multipath fading.

In broadband wireless access systems BWA adaptive transmit power control is required to equalize near/far and rain fading effects so that the received powers at the hub from multiple subscribers arrive at almost the same power level. An equal received power level is desirable for three reasons:

- For TDMA systems, equal power allows burst receivers to more easily recover successive signals from different subscribers.

- For FDMA systems, equal power minimizes the required adjacent-channel rejection of the hub receiver and minimizes the required adjacent-channel spurious emission suppression required of the upstream transmitters.

- For systems that employ intensive frequency re-use, such that the upstream channel is spatially reused at the same hub in another sector, equal received power minimizes the required hub antenna out-of-sector rejection required to ensure adequate $\mathrm{C} / \mathrm{I}$.

Many authors have studied power control issues so far. A variety of power control schemes have been put forward based on different criteria. Power control schemes have two loops, inner loop and outer loop. Furthermore, the inner loop can be classified as open loop control and closed loop as shown in Figure 3.2. 


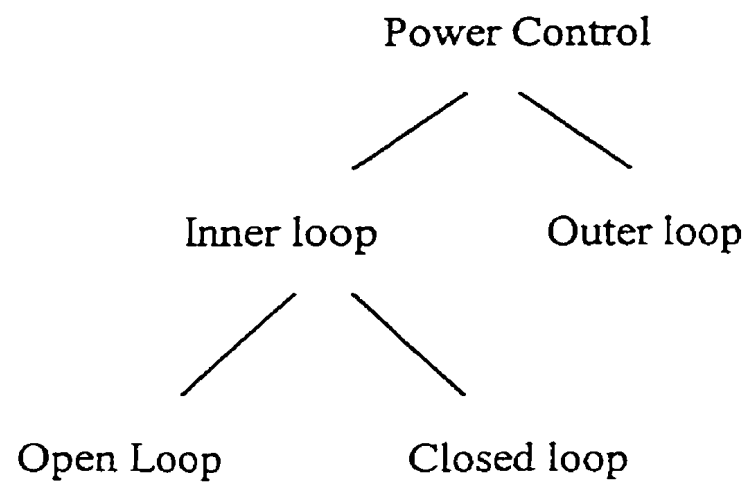

Figure 3.2 Power control loops

\subsubsection{Inner Loop Power Control}

Inner loop power control can be divided into two categories: the open loop and the closed loop power control methods. In the following subsection we will explain the difference between them.

\subsubsection{Open Loop Power Control}

In open loop power control, the transmitter attempts to estimate the path loss based on the measurement of the receiving power. It adjusts the transmitted power according to the received forward link signal strength. Reception of a strong signal indicates that the transmitter and receiver are either very close or there is a good propagation path between transmitter and receiver, hence a relatively low power is needed for acceptable reception.

The open loop power control is based on the assumption that the path losses on both forward link and reverse link are the same. Hence, it mainly compensates for the path loss and shadowing. However, due to the discrepancy between the carrier frequency bands for each link, although lognormal shadowing normally exhibits reciprocity, the fading is independent for the reverse link and forward link, and the path losses will not be 
the same for each link. The coarse adjustment of the transmitting power needs to be further refined. This is accomplished by closed loop power control.

\subsubsection{Closed Loop Power Control}

Closed loop power control is a sort of fine-tuning on the open loop power control. In cellular systems, taking the reverse link power control as an example, the mobile transmitted power is controlled by a signal from the base station. According to the power level assigned by system controller, each base station controller maintains the desired power level for each mobile that is active within that cell.

The subscriber uses two frequencies, one for transmission and one for receiving. The frequency separation has very important implications for the power control process. It causes the multipath fading to be independent in both links, due to the frequency separation, i.e. the mobile can not measure the path loss of a received signal and assume that the same instantaneous path loss is present on the reverse link. To account for the independence of multipath fading on the forward link and the reverse link, the mobile will be controlled by power adjustment commands from the base station. The base station measures the received signal level from each mobile. The measured signal is compared with a desired signal level and a power command $C_{p}$ is sent through feedback channel to that mobile to adjust its transmitted power. In reality, it is often desired to combine the open loop and closed loop power control techniques [29].

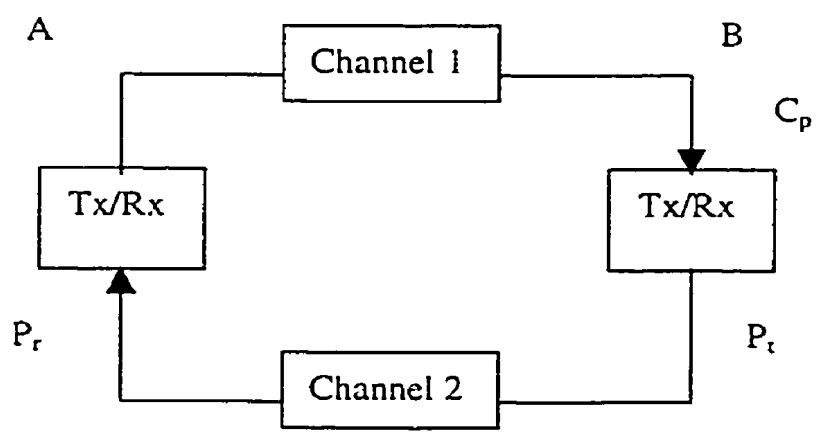

Figure 3.3 Closed loop Power control 
The rate of the power command must be high enough to track the multipath fading. It is important that the latency in determining the power control command and the transmission process time should kept small so that the channel conditions will not change significantly before the power command can be received and executed.

While the closed loop control may not be fast enough to quite keep up with fast fading, it is at those higher fading rates that coding and interleaving are most effective. At lower fade rates the interleaving may be less effective, but then power control is extremely robust.

\subsubsection{Outer Loop Power Control}

The closed loop power control also employs what is termed an outer loop power control. This mechanism ensures that the power control strategy is operating correctly. The frame error rate at the base station is measured and compared with the desired error rate. If the difference between error rates is large, then the outer loop power command threshold is adjusted to yield the desired FER. Increasing the threshold reduces the frame error rate, thereby improving the quality of the received signal. Reducing the threshold tends to increase the frame error rate.

\subsection{Diversity}

Fading is considered to be one of the main causes of performance degradation in a mobile radio system [17]. Fading counteraction is usually carried out by means of diversity methods. Diversity is a powerful communication receiver technique that exploits the random nature of radio propagation to find independent or at least highly uncorrelated signal paths for communication. If one path undergoes a deep fade, another path may have a strong signal. By having more than one path to select from, both the instantaneous 
and average SNRs at the receiver may be improved, often by as much as $20 \mathrm{~dB}$ to $30 \mathrm{~dB}$. [18].

\subsubsection{Long term fading counteraction}

The methods of counteracting the lognormal shadowing use macroscopic diversity. Macroscopic diversity is simply implemented by providing more than one base station so that the mobile always have a clear radio path to one base station. This type of diversity can be considered as a kind of space diversity.

\subsubsection{Short term fading counteraction}

Methods of counteracting fast fading use microscopic diversity. Microscopic diversity relies on the fact that independent signals have a low probability of experiencing deep fades at the same time.

Diversity techniques can be divided into:

- Space or antenna diversity is one of the most popular forms of diversity used in wireless communications. Antennas separated by half wavelength or more will have uncorrelated envelopes. While one antenna sees a signal null, another antenna may see a good signal. One of the advantages is that it does not need more frequency spectrum. On the other hand, its drawback is that it needs a sufficient space separation between antennas.

- Polarization diversity takes advantage of the orthogonality of the polarized wave components of the travelling wave. Although the two components of the polarized wave travel through similar paths, the obstacles encountered by these waves scatter each one in a different way. It has been primarily used for fixed radio links, which vary slowly in time. For example, in line-of-sight microwave links it can support two simultaneous users on the same radio channel. 
Note that with the polarization only two antenna branches are available, as opposed to space diversity where several branches can be obtained using multiple antennas.

- Frequency diversity transmits information on more than one carrier frequency. The frequency separation between carriers should be larger than the coherence bandwidth. This technique has the disadvantage that it requires large bandwidth.

- Time diversity transmits information at time spacing that exceeds the coherence time of the channel, so that multiple repetitions of the signal will be received with independent fading conditions. Time diversity is usually exploited via interleaving and FEC. One drawback with time diversity is the delay which arise due to time needed to collect the repeated transmission. Also, increased bandwidth is required.

- Angle diversity is used where two or more directional antennas can be pointed at different directions at the receiving site. For example, an adaptive antenna tracks the best signal by adaptively positioning itself to the best angle.

\subsection{Outage Probability and System Availability}

Outage probability is a useful performance measure, expressing the fraction of time that the signal-to-interference ratio is below a certain threshold, due to fading, for a given desired user in a given position. If noise is also significant, an outage occurs when the signal-to-interference-plus-noise ratio (SNR) drops below the threshold.

The receiver threshold can be called protection ratio or target SINR as well. Its value depends on the required bit error probability (BER) for a specific modulation.

The outage probability for a particular subscriber can be expressed by 
$P_{\text {outuge }}(\gamma)=\operatorname{Pr}\{\operatorname{SINR}<\gamma\}$ for that subscriber,

where $\gamma$ is the threshold.

System availability can be defined as the percentage of subscribers position having less than $1 \%$ outage. 


\section{Chapter 4 \\ LMCS SIMULATION MODEL}

In this chapter, the LMCS system model to be implemented is thoroughly described and all related assumptions are clearly stated and justified. The objective of this simulation is to investigate the system availability for fixed users by implementing power control in LMCS. The effects of system parameters such as the characteristics of propagation environment, power control command rate and power control step sizes are considered. Simulation is conducted for reverse link (up link) only.

\subsection{Simulation Model}

Observations from [8] and [30] indicate that strong signals are received only at locations where a LOS path was available between the transmitter and receiver. Both studies concluded that a LOS path is required to provide reliable LMCS service. Line of sight (LOS) system performance will be investigated through simulations. LMCS systems attempt to reuse the allocated frequency band in each cell by means of directional antennas and polarization reuse in adjacent sectors. Perfect orthogonal polarization is assumed which means perfect isolation between horizontal and vertical polarization.

\subsubsection{System Model}

The LMCS simulation model is designed to have a cellular layout with frequency reuse factor of 4 (i.e. total frequency band is reused in each of 4 sectors). We assume that 9 cells with square grid cover the service area; each hub consists of 4 sectors.

Highly directional subscriber antennas and perfect orthogonal polarization are used to reduce the cochannel interference and to provide high coverage. As we can see from Figure 4.1, $\mathrm{V}$ and $\mathrm{H}$ refer to vertical and horizontal frequency polarization, respectively. 
Therefore, two of the sectors in each cell will have horizontal polarization and the other two sectors will have vertical polarization. Cells are assumed to be square and the base station of each cell is located at the center of the cell.

The cell radius is $2 \sqrt{2} \mathrm{~km}$; i.e. the distance between base stations is $4 \mathrm{~km}$. This gives a total coverage area of $144 \mathrm{~km}^{2}$. Subscribers will be uniformly distributed in each sector of the cell. For every subscriber signal received at the base station, there will be up to 17 interferers. In order to neglect the border effect we collect data for users in the central cell. As part of an overall program to evaluate power control, we have developed a simulation model.

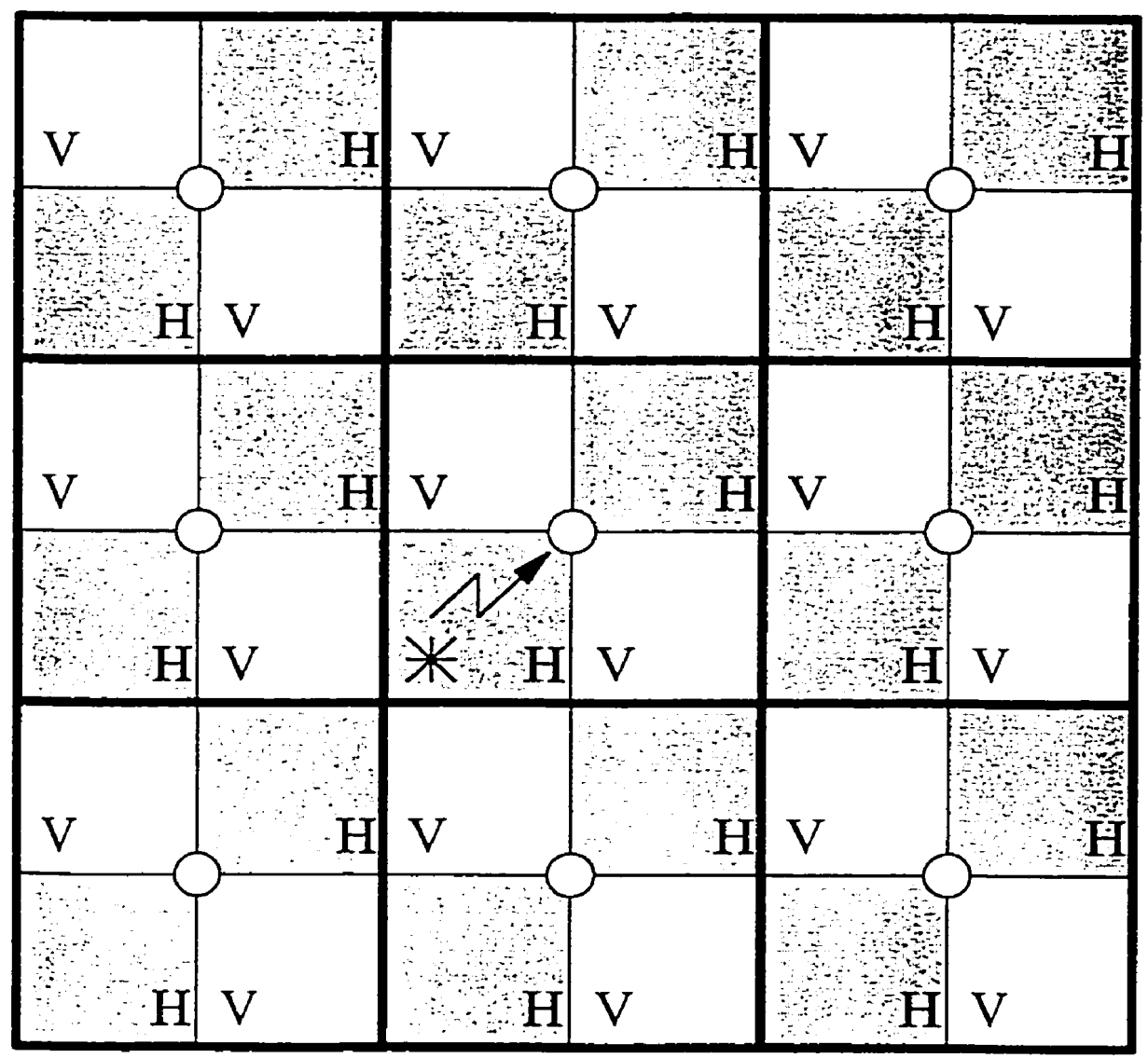

Figure 4.1 LMCS System Model 


\subsubsection{Propagation Model}

As mentioned in chapter 3, a transmitted signal in radio channel is subjected to a propagation path loss directly proportional to the propagation exponent, shadowing and multipath fading. The effect of propagation environment parameters such as Rician $K$ factor, propagation exponent and shadowing standard deviation on system availability will be studied. Based on propagation studies in the LMCS project, simple but realistic assumptions are made.

\subsubsection{Path Loss Model}

In mobile communication systems a reference distance is used to represent the minimum distance allowed between the transmitter and the receiver. Here we are going to denote this distance by $d_{o}$ and assume it to be 20 meters. See equation (3.4)

Now, we can calculate the average received power at a base station with distance $d$ from the fixed subscriber $\left(d>d_{o}\right)$ by the following equation

$$
S=P_{T} G_{T} G_{R}\left(\frac{c}{4 \pi f d_{0}}\right)^{2}\left(\frac{d_{o}}{d}\right)^{n}
$$

where $P_{T}$ is the transmitted power of subscriber, $f$ is the carrier frequency in $\mathrm{Hz}, n$ is the propagation exponent, $G_{T}$ and $G_{R}$ are the gains of the transmit and receive antennas, respectively, and $c$ is the speed of light $\left(3 * 10^{3} \mathrm{~m} / \mathrm{sec}\right)$. For directional antennas the gain could be the main lobe gain or the side lobe gain based on the location of the subscriber location within the cell.

One of the simulation parameters is the path loss exponent. Typically, the path loss exponent for free space propagation is 2 and for shadowing urban areas it is 3 to 5 . The likelihood that a channel link between a fixed subscriber and its base station to have a 
path loss exponent equal to 2 is higher in comparison to that between a fixed subscriber and other base stations.

\subsubsection{Large Scale Fading (Shadowing)}

As we noticed from chapter 3 , the received power for fixed users at the same distance from the transmitter but at different locations will be different. This phenomenon is known as shadowing. Large-scale fading or shadowing is considered in this model as another channel impairment that affects the transmitted signal and can be represented by a lognormal distribution with distance dependent mean as in equation (4.1) and a certain standard deviation $\sigma$.

\subsubsection{Small Scale Fading}

In our simulation model, we included the multipath fading. Different statistical distributions are used in mobile communications to represent such channel impairments. Results in [11] study indicate that the existence of a LOS path is crucial to alleviate the coverage problem and reduce outage probability for LMCS systems.

The existence of a LOS path plus the scatter component can be represented by a Rician distribution. Referring to what we discussed in chapter 3, Rician distributions can be represented by a Rician $\mathrm{K}$ factor, which is the ratio of the deterministic LOS component to the scattered component. It can be represented by the following equation

$$
K=\frac{A^{2}}{2 \sigma^{2}}
$$

When $\mathrm{K}=0$ the deterministic component (LOS power) goes to zero and the distribution becomes Rayleigh fading. In our simulation we will study the effect of the Rician $\mathrm{K}$ factor on the outage performance. We will assume different propagation environments for the desired subscriber and the interferers. The user of interest will have a better link to its 
base station, so a higher value of $\mathrm{K}$ is used to simulate this situation. On the other hand, links between the user and other cells will have lower $\mathrm{K}$ values.

In order to generate a Rician fading channel we have to generate two independent normal distribution samples with zero mean, a certain standard deviation, and a LOS component $A$ as shown in the following equation; for $\beta$, the envelope of the Rician faded signal:

$$
\beta=\sqrt{\left(A+U_{X}\right)^{2}+U_{Y}^{2}}
$$

In the above, $U_{X}$ and $U_{Y}$ are Gaussian r.v's with zero mean and standard deviation $\sigma_{U}$. We will assume a certain value for the subscriber Rician $\mathrm{K}$ factor, then we calculate the LOS component by solving eq 4.2 and the mean squared value of $\beta$, which is $E\left(\beta^{2}\right)=2 \sigma^{2}+A^{2}$. The average received power is set to 1 (i.e. unit average received power). The solution for the two equations will give

$$
\sigma=\sqrt{\frac{1}{2+2 K}} \quad \text { and } \quad A=\sqrt{\frac{2 K}{2+2 K}}
$$

Substituting in equation 4.3 gives the Rician parameter.

Propagation studies in [11] show that the channel will remain approximately time invariant or time flat for $0.01 \mathrm{sec}$ to $0.05 \mathrm{sec}$. As the expected data rate for LMCS is 20 $40 \mathrm{Mbps}$ it is obvious that the signal will undergo slow fading relative to the bit rate. The slow fading environment allows a closed loop transmit power control system to operate accurately and track multipath fading.

A channel model that is time correlated is developed to provide a more realistic performance analysis compared to independent fading assumptions. The samples can be made correlated in time by passing the independent Gaussian distributed samples $\mathrm{X}$ and 
Y through the low pass filter shown in Fig 4.2. The time correlated samples can be calculated as follows:

$$
\begin{aligned}
& U_{\text {Xnew }}=\alpha * U_{\text {Xold }}+(1-\alpha) * X \\
& U_{\text {Ynew }}=\alpha * U_{\text {Yold }}+(1-\alpha) * Y
\end{aligned}
$$

Where $\mathrm{X}$ and $\mathrm{Y}$ are two independent Gaussian distributions with zero mean and standard deviation $\sigma$ and $U_{x}$ and $U_{y}$ are the time correlated samples. Also, $\alpha$ denotes the correlation factor. If $\alpha=0$ then the samples are uncorrelated and thus independent and when $\alpha=1$ the time samples are identical.

In order to avoid the transient response of the low pass filter, we start collecting time correlated samples after the steady state is reached. The number of independent samples that we need to generate before it reaches steady state depends on the ratio between the time correlated variance and the independent variance. If we denote the time correlated variance as $\sigma_{U}^{2}$ and the independent samples variance as $\sigma^{2}$, then we can define the ratio between these variances at steady state as follows

$$
u=\frac{\sigma_{U}^{2}}{\sigma^{2}}=\frac{1-\alpha}{1+\alpha}
$$

The time constant of the filter is $1 / 1-\alpha$, and it is assumed that the steady state is achieved within 3 time constants (this would correspond to 30 samples for $\alpha=0.9$ ). At the steady state, the independent $X$ and $Y$ samples variance has to be modified in order to satisfy the unit average received power constraint : $\sigma^{2}=\frac{1}{u} \frac{1}{2 K+2}$, while the correlated samples $U_{X}$ and $U_{Y}$ will have a variance of $\sigma_{i}^{2}=\frac{1}{2 K+2}$ 


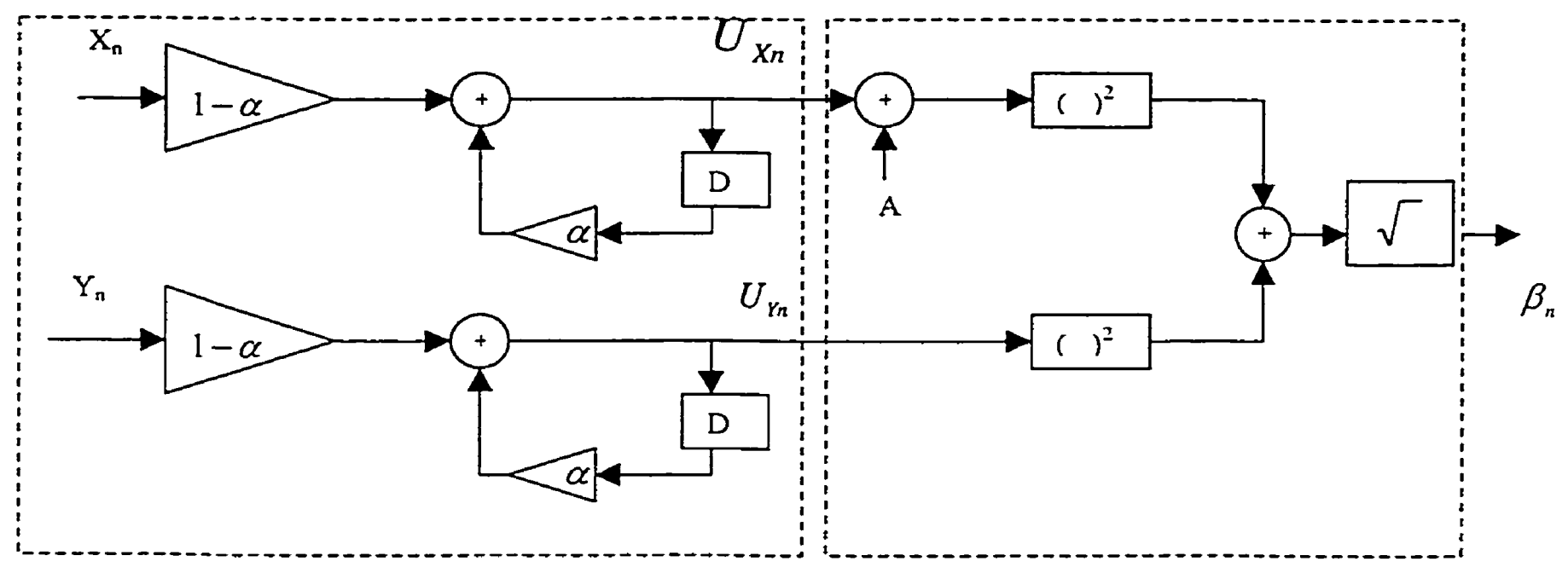

Fig 4.2 Time correlated Rician Fading

Refering to Figure 4.3, we plot the average received power $\left(\beta_{n}^{2}\right)$ in linear and $\mathrm{dB}$ scale for different values of $\alpha$. It can be seen that the channel is slowly varying in time with a correlation factor $\alpha=0.9$ (i.e. high correlation between samples) compared to the channel with no correlation $(\alpha=0)$. 


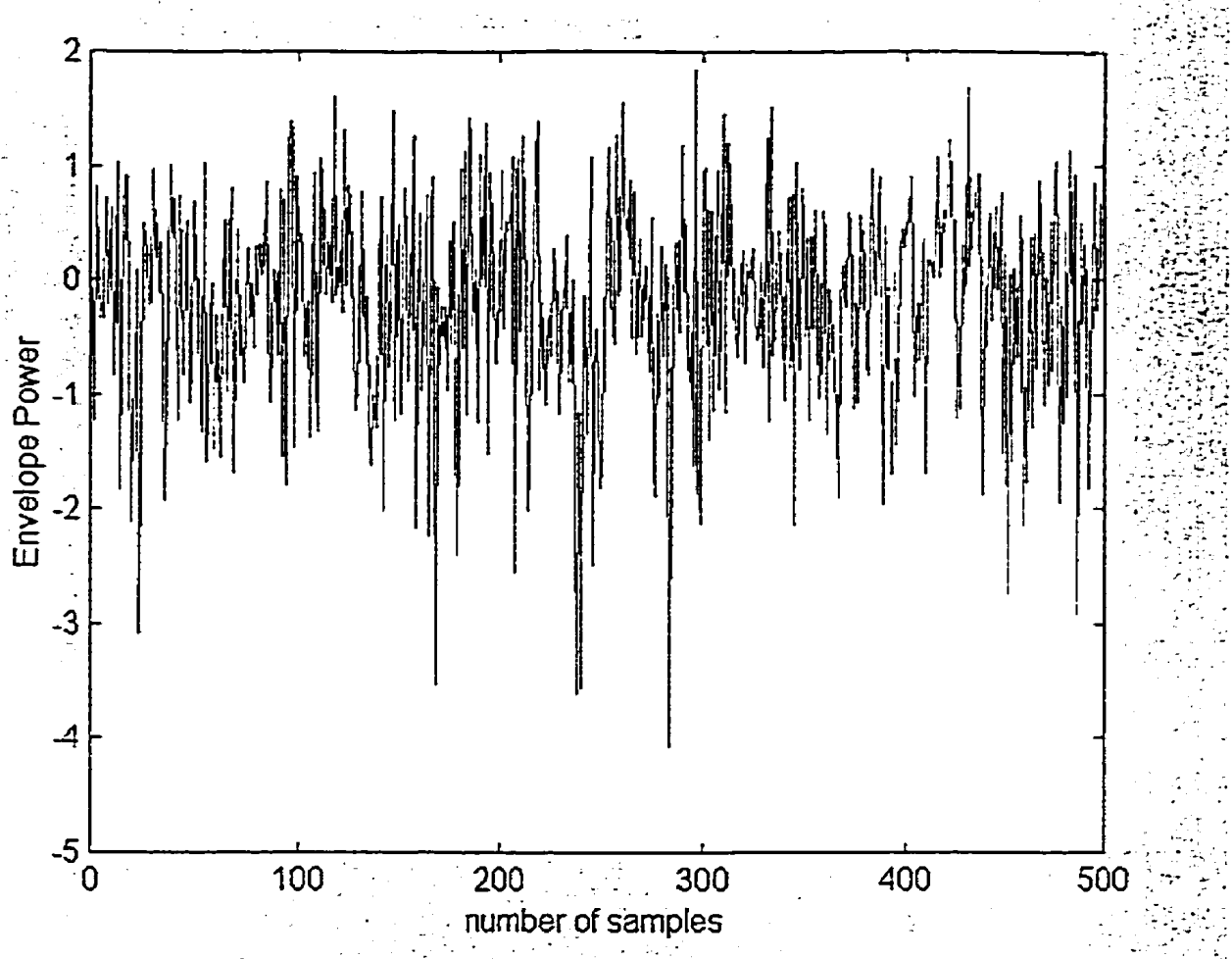

(a) $\alpha=0[\mathrm{~dB}]$

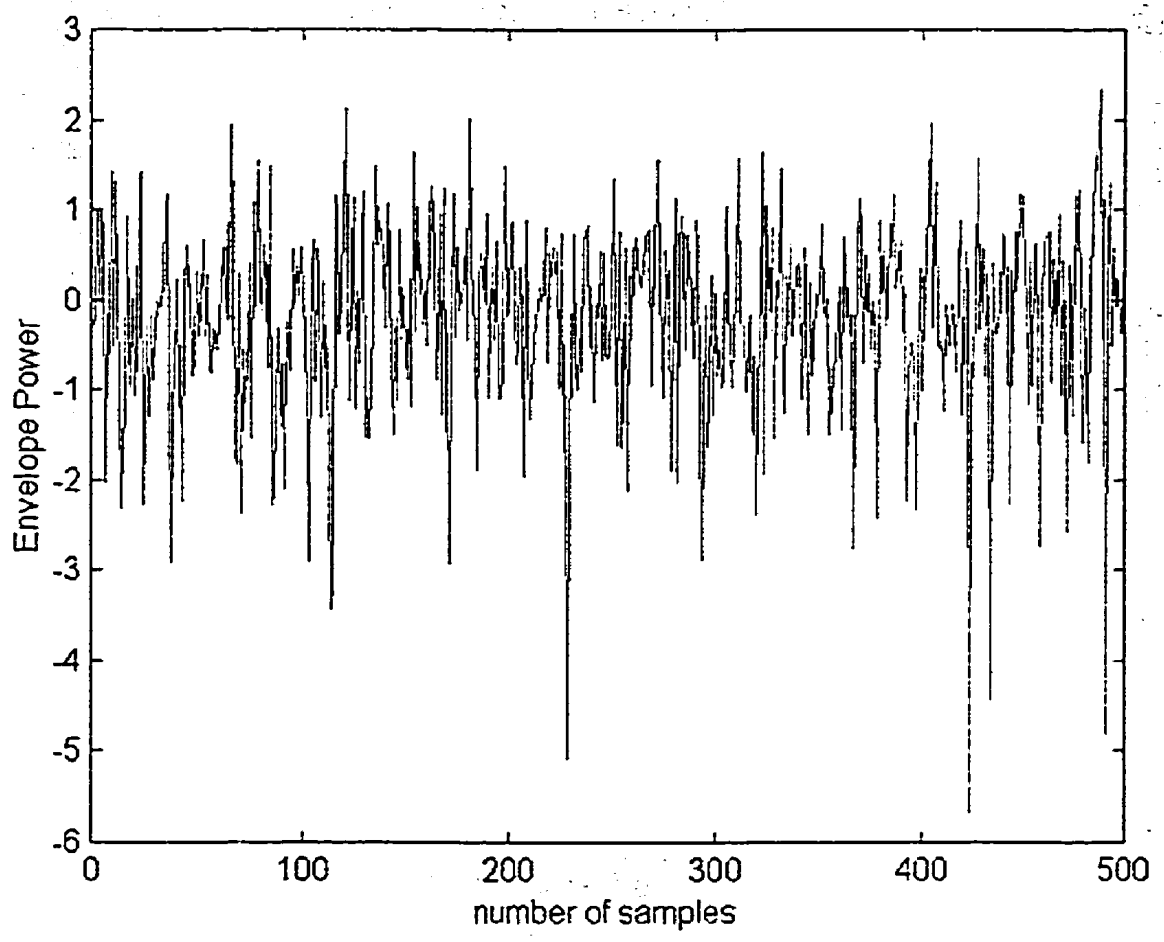

(b) $\alpha=0.2[\mathrm{~dB}]$ 


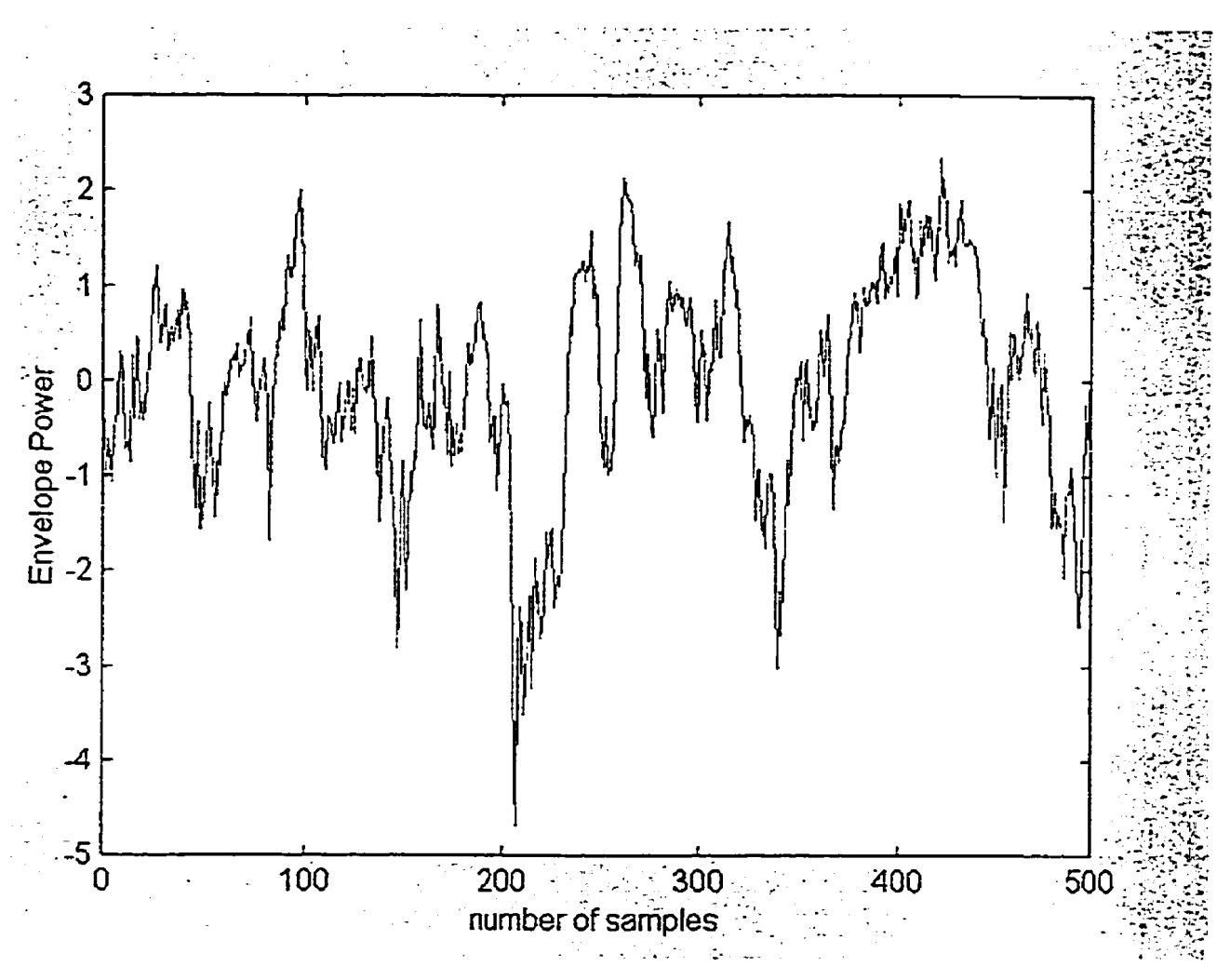

(c) $\alpha=0.9[\mathrm{~dB}]$

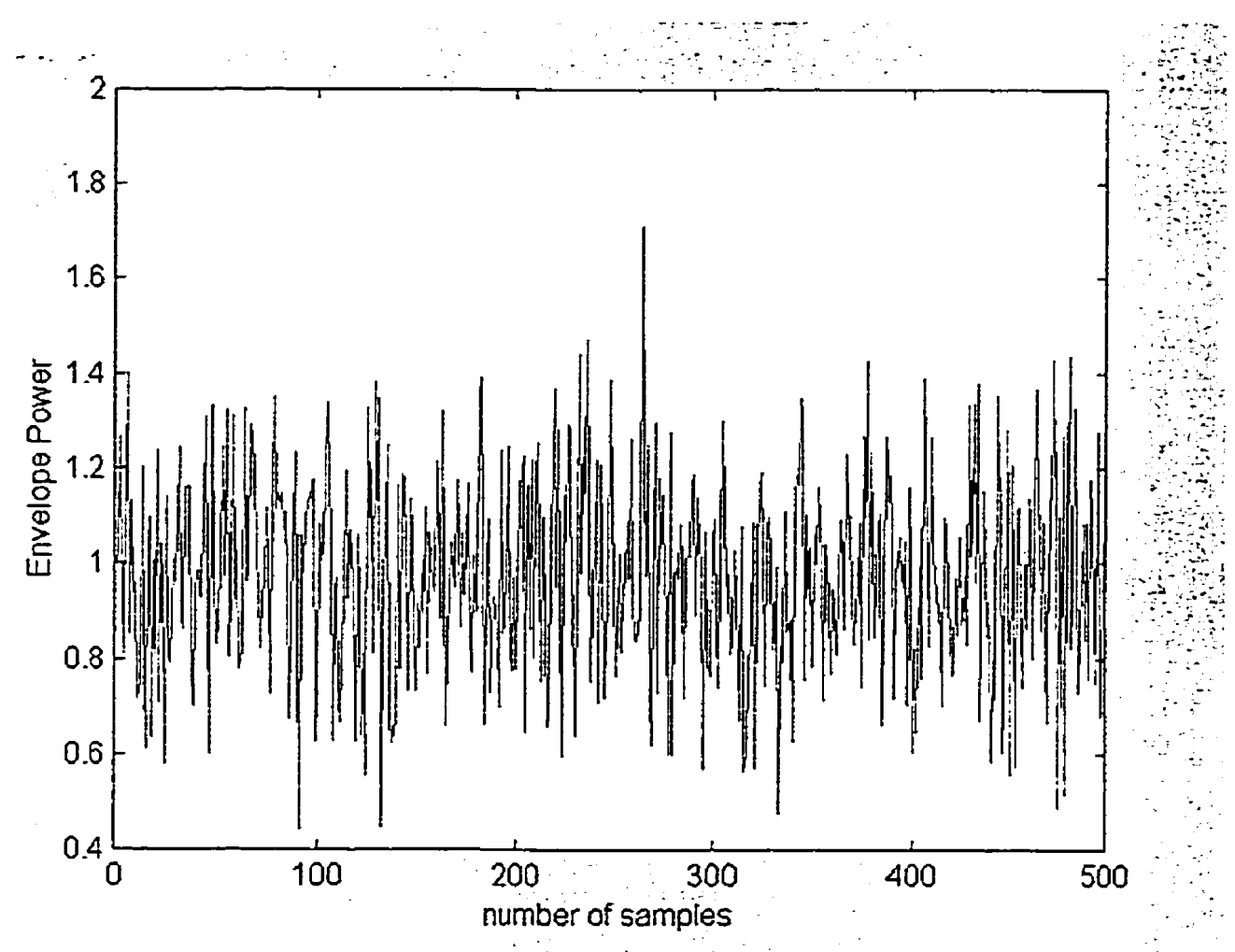

(d) $\alpha=0.0$ [Linear scale] 


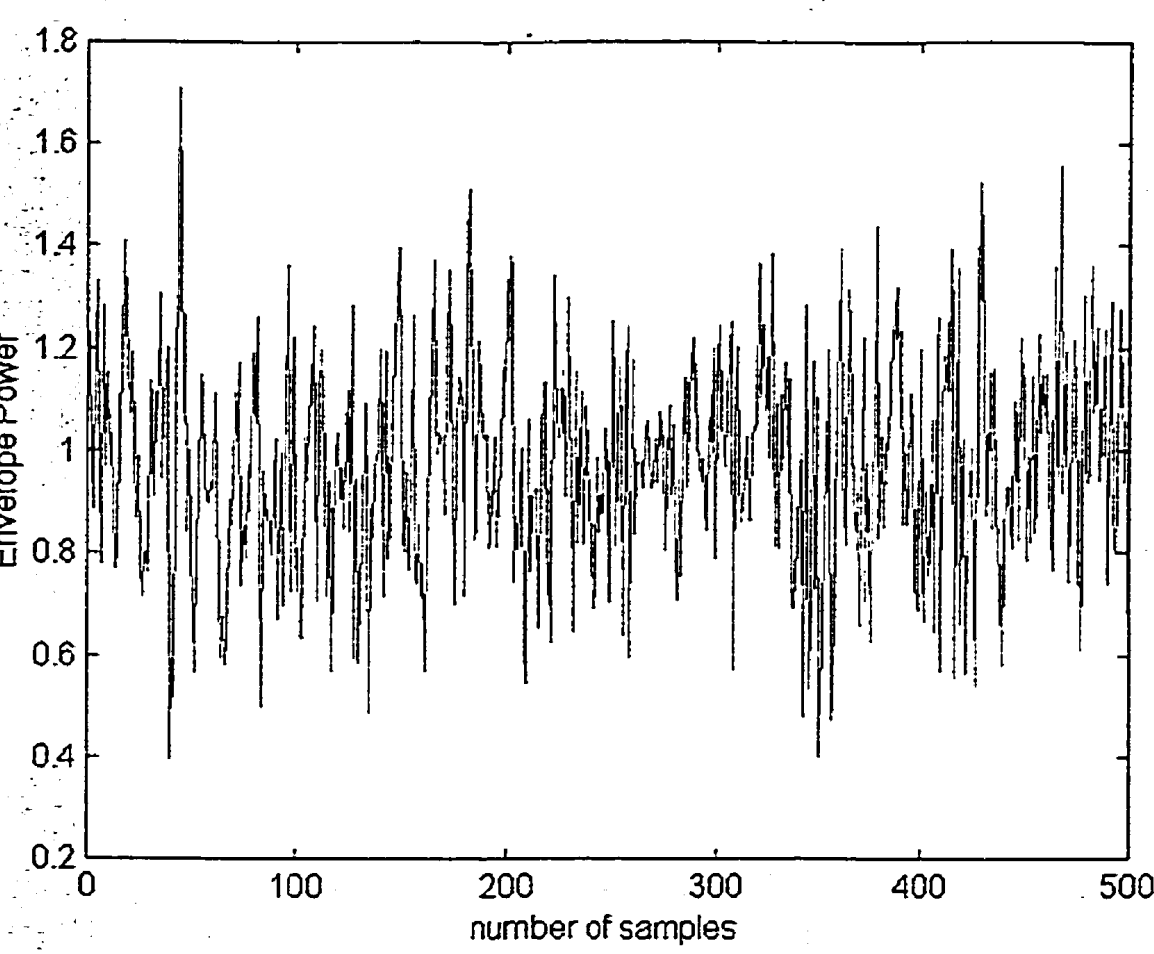

(e) $\alpha=0.2$ [Linear scale]

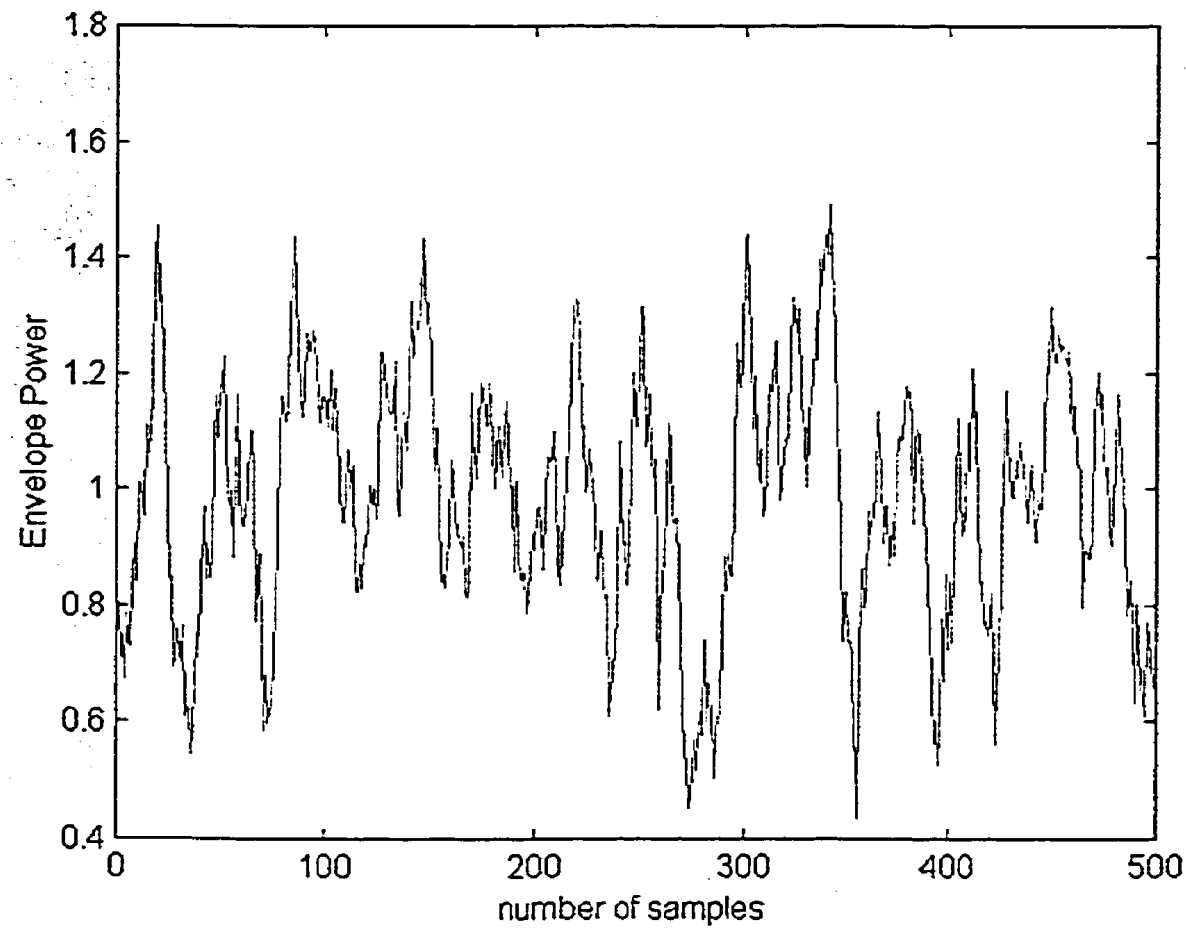

(f) $\alpha=0.9$ [Linear scale]

Figure 4.3 Envelope power of a Rician channel with different correlation factors. 
Now the received power by the base station, which was given by equation (4.1), can be modified to include the impact of shadowing and multipath fading as follows:

$$
S=P_{T} G_{T} G_{R}\left(\frac{c}{4 \pi f d_{0}}\right)^{2}\left(\frac{d_{0}}{d}\right)^{n} 10^{\frac{x}{10}} \beta^{2}
$$

\subsection{System Parameters}

It has been shown that directional antennas can improve the performance of LMDS systems [9]. In this section we'll review some of the system parameters such as the dynamic range of the transmitted power and thermal noise. The first two parameters can be controlled, whereas the last one depends on the channel bandwidth. In the previous work [9], the gain ratio of the directional antenna has been established. So, in this work we'll use the same value.

\subsubsection{Directional Antennas}

Directional antennas or sectored antennas are used to reduce the received interference through the antenna main lobe by attenuating out-of-beam interferers. Unlike mobile cellular systems, in LMDS/LMCS the subscriber antennas are highly directional and point toward one specific base station. It is shown in [9] that LMCS system outage performance is much better with directional antennas compared to the case of omnidirectional antennas. The LOS system performance is more sensitive to the hub antenna gain ratio and could be dramatically improved when using a directional antenna of high gain ratio at the subscriber. Therefore, in our simulation directional antennas were used at both subscriber and base station.

The main lobe gain is uniform and has a certain value; the side lobe gain will have a uniform gain but with a lower value than the main lobe gain value. The difference (in $\mathrm{dB}$ ) between these values is the gain ratio of the antenna. It is fixed for both the base and the user at $25 \mathrm{~dB}$. The main lobe gain of the base station is assumed to be $25 \mathrm{~dB}$ and the 
sidelobe gain is $0 \mathrm{~dB}$. On the other hand, the subscriber main lobe is $35 \mathrm{~dB}$ and the sidelobe is $10 \mathrm{~dB}$. Each cell will have 4 base station antennas with beamwidth of 90 degree. Each user will have a highly directional antenna with beamwidth of 3 degrees.

\subsubsection{Dynamic Range}

The range of transmitted powers limits power controls. Dynamic range may be limited further by terminal capabilities and need to accommodate thermal noise. We assumed different scenarios for the subscriber's dynamic range. The transmitted power is either bounded by upper and lower limits, or it could be bounded by just one of the limits. The value of the upper and lower bounds are chosen to be $-10 \mathrm{dBw}$ and $-50 \mathrm{dBw}$. The upper bound for the transmitted power is a very important factor. For example, a user which has a high outage probability (i.e. low received SINR) will try to increase its transmitted power in order to satisfy the base station requirement and will cause severe interference to the other subscribers. Furthermore, the upper bound reflects the fact that the maximum power output from any practical transmitter is limited. Therefore the upper bound is crucial for satisfactory performance.

\subsubsection{Thermal Noise}

The receiver thermal noise power assumed here to be additive white Gaussian noise (AWGN) and can be calculated from the following equation

$$
N=K T B F
$$

Where $\mathrm{K}$ is Boltzmann's constant $\left(1.38 \times 10^{-23} \mathrm{Joules} / \mathrm{Kelvin}\right), \mathrm{T}$ is the system temperature $(290 \mathrm{~K}), \mathrm{B}$ is the channel bandwidth in $\mathrm{Hz}$ and $\mathrm{F}$ is the noise figure. The noise figure is assumed to be $8 \mathrm{~dB}$ in this study [9].

It is suggested that the uplink channel is $2 \mathrm{MHz}$ and the downlink channel is $40 \mathrm{MHz}$ [7]. 


\subsection{Binary SINR-Based Power Control}

SINR-based power control model was investigated in [1,2]. It is an example of the closed loop technique. In chapter 3 , we discussed different power control approaches. Measuring the received signal or SINR can implement these algorithms. It is obvious that SINRbased power control is more realistic and is actually desirable from the radio link performance perspective, since it is SINR that determines the received bit error probability. This power control algorithm allows the power commands to be updated at a higher rate than the multipath fading rate. This type of power control can partially accommodate the effects of fast fading [1].

The performance of the reverse link (uplink) will be simulated. The base station measures the received SINR from desired user and compares it with a SINR threshold. The difference between received SINR and the outer loop SINR threshold can be defined as follows

$$
e=\gamma_{\text {received }}-\gamma_{\text {th }}
$$

If the error is greater than zero, a power command is issued by base station for the transmitter to decrease its power by a predetermined amount, we refer to this amount as the step size. Otherwise an up command is sent to increase the transmitted power.

The user's transmitted signal power $P_{i}(\mathrm{dBw})$ is updated by fixed step size of $\Delta(\mathrm{dB})$. During $i$ th period, the signal power received at the base station is $P_{i}+G$, where $G$ is the channel gain in $\mathrm{dB}$. The interference from co-channel users are added linearly to thermal noise, converted to $d B$, and then subtracted from the received signal's power in $d B$ to give the measured SINR at the base station:

$$
\gamma_{\text {received }}=\frac{S}{\sum_{i=1}^{17} I_{i}+N_{u}}
$$




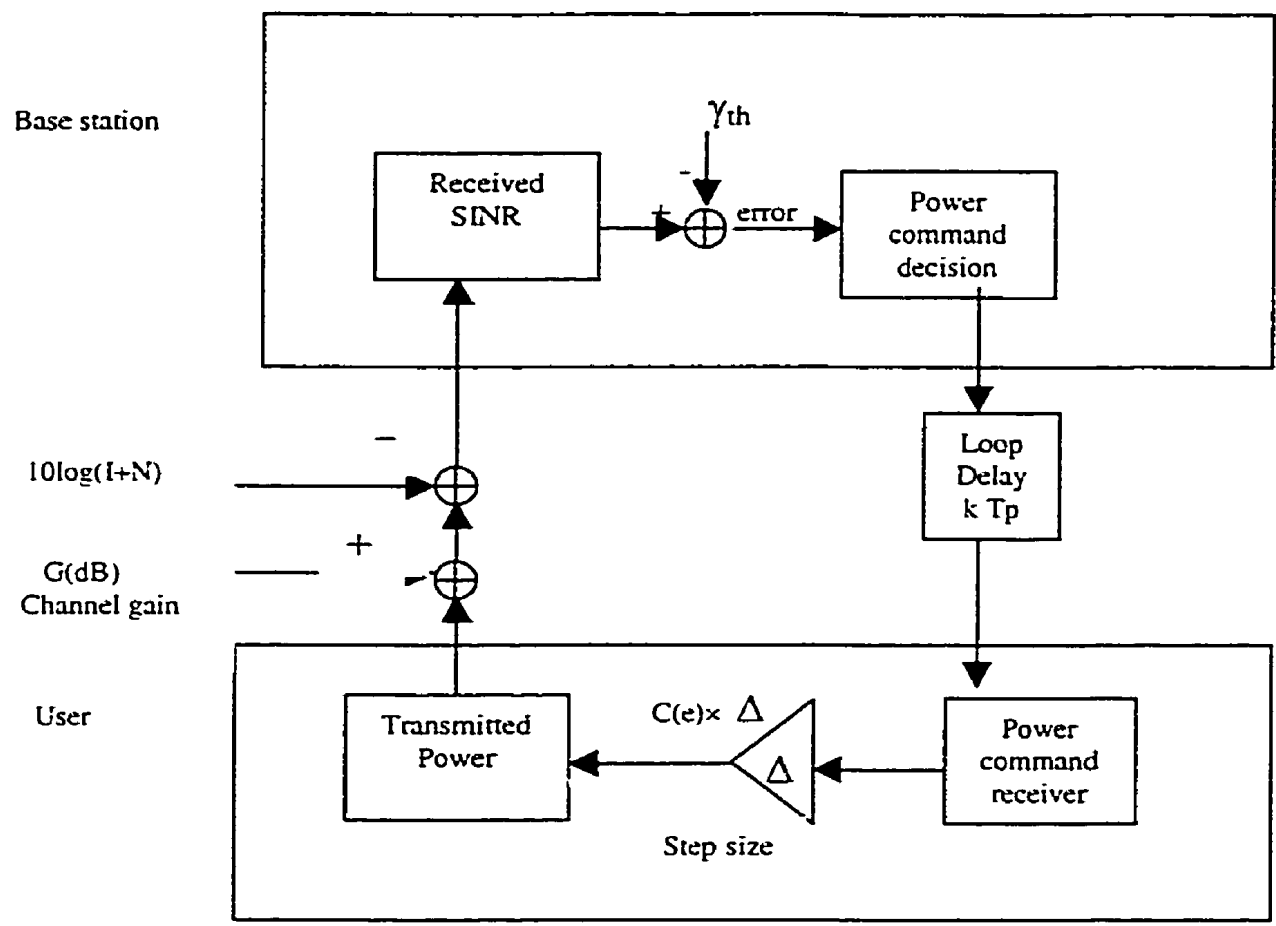

Figure 4.4 SINR-based power control

The measured SINR is compared with the desired level $\left(\gamma_{\mathrm{th}}\right)$ e.g. $15 \mathrm{~dB}$. The difference between these two values is quantized by the power command decision and a power command is transmitted to the user through the feedback channel.

$$
\begin{aligned}
& \mathrm{P}_{\mathrm{i}+1}=\mathrm{P}_{\mathrm{i}}-\mathrm{C}(\mathrm{e}) \cdot \Delta \\
& C(e)=\left\{\begin{array}{cc}
-1 & \text { if } e \leq 0 \\
1 & \text { if } e \geq 0
\end{array}\right.
\end{aligned}
$$

where $C(e)$ is the power control command. 
The performance of the system depends greatly on how the power control threshold $\left(\gamma_{\mathrm{th}}\right)$ is set. We have assumed a fixed outer loop power control threshold for all users; this value can be adjusted according to the frame error rate FER. The effect of power control command error is not included in this study.

In our simulations, power control is applied for the entire system. However, the data are collected only for users within the first sector of cell 5 (refer to figure 4.1). This is due to the fact that the interference for radio links of the user at the edge cells of our system model is different from those at the central cell.

The system stability was carefully studied, and tests were applied to confirm whether the system becomes stable or not. We noticed that the system always reached steady state and remained stable after the initial startup period, which was always less than 40 power control sampling periods. The startup period data is excluded from our results.

Imposing an upper bound in the transmitted power by each user, i.e. setting the dynamic range is very important. Thus, we study the effect of the dynamic range. The upper bound will force the user not to go beyond a maximum value in order to prevent causing more interference to other users. For example, a user who has poor quality (i.e. low SINR) will try to transmit with a higher power level in order to be received at the corresponding base station, and this yield more interference to other users, which in turn will increase their power in order to maintain their quality. This positive feedback can be reduced by imposing maximum transmitted power.

Simulation results presented in $[1,2]$ indicates that, for feedback SINR based power control to work effectively against fast multipath fading with diversity, the power command updating rate must be significantly higher than 10 times the multipath fading rate. At the same time power command rate must be slow compared to data bit rate so that power command bits do not consume much available bandwidth. 


\subsection{Multi-step SINR-based Power Control}

A multistep power control mechanism for uplink communications to improve the system performance was first suggested in [3]. In the multi-step power control algorithm, the receiver, which is the base station in the case of up link, issues its power control command to the subscriber to update the transmitted power. The power control command quantizes the difference between the received SINR and the desired level set at the base station into multiple levels.

The functional block diagram of the multi-step SINR based power control method is shown in Fig. 4.5, and we can describe its operation as follows:

1. The power control of each uplink is assumed to operate synchronously and periodically with power control sampling period $T_{p}$.

2. At the beginning of a control sampling period, each base station measures the received SINR (dB) and compares with a pre-defined desired level $\left(\gamma_{t h}\right)$. The difference is denoted by error.

3. The base station encodes the difference error into a power control command $\mathrm{cmd}$ in the block of the power control command decision and sends cmd to the subscriber. The cmd is a multiple-level coded command. The power control method is said to be in mode- $n$ if $c m d$ has the range of $\{-n,-(n-1), \ldots,-1,0,1 \ldots n-1, n\}$ and in mode-0 if cmd is either 1 or -1 . Note that mode-0 is the SINR based power control scheme.

4. The subscriber performs the $c m d$ by the power control command received.

5. The subscriber updates the transmitted power by an amount of $\mathrm{cmd} * \Delta$ at the beginning of the next sampling period.

The encoding of the error into power control command $c m d$ can be explained as follows; we define key $=$ error $-\Delta(\mathrm{dB})$, then the cmd command can be related to key according to the following equation. 


$$
c m d=\left\{\begin{array}{ccccc}
-n & \text { if } & k e y & \in & (n-0.5, \infty) \\
& \cdot & & & \\
-1 & \text { if } & k e y & \in & (0.5,1.5] \\
0 & \text { if } & k e y & \in & (-0.5,0.5] \\
1 & \text { if } & k e y & & (-1.5,-0.5] \\
& \cdot & & & \\
n & \text { if } & k e y & \in & (-\infty,-n+0.5]
\end{array}\right\}
$$

It is found in [3] that there is an appropriate control mode for a given step size, and a high power control mode is unnecessary since sending more power control bits will consume too much available bandwidth. Another important result is the existence of an optimal step size. The desired level $\left(\gamma_{t h}\right)$ should be selected carefully. Setting the desired level too high or too low will significantly degrade the system performance.

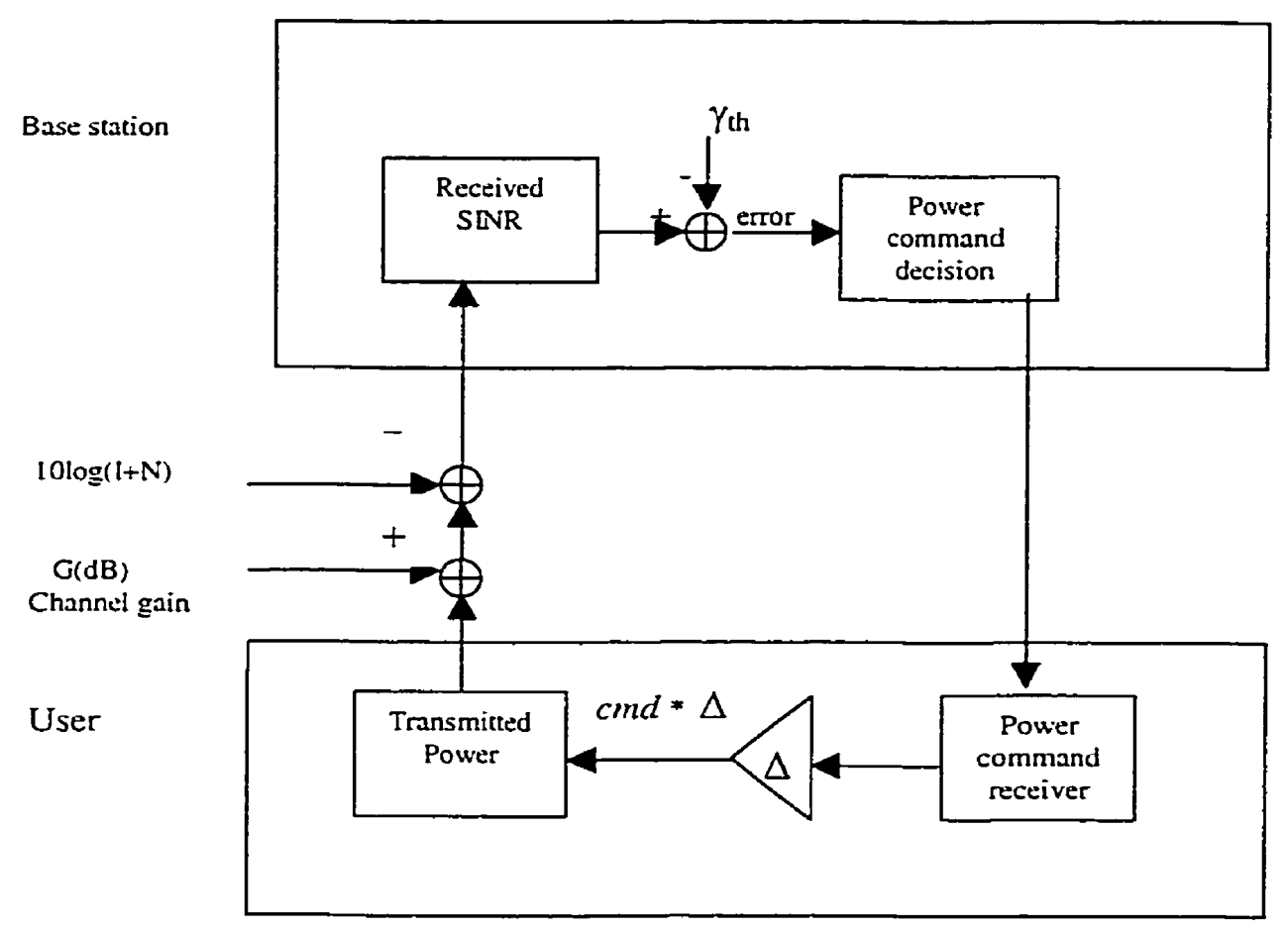

Fig 4.5 Block diagram of the multi-step power control 


\subsection{Simulation Algorithm}

The flow diagram of the simulation program is shown in Fig 4.6 and the framework of the simulation program is given below:

Step 0: Set up system parameters

- Set up system parameters such as cell radius, uplink/downlink channel bandwidth, and antenna gains and beamwidth.

- Set up the environment parameters such as propagation exponent $\mathrm{n}$ for the desired subscriber and interferer, the Rician $K$ factor for intercell users and intracell user, lognormal shadowing mean and standard deviation values and the correlation factor $\alpha$.

- Set up the power control parameters such as SINR outer loop threshold, and number of samples per location, dynamic range, PC step size and number of cycles (i.e. number of subscribers).

- Set up the system threshold for the outage probability calculation for the subscriber of interest.

Step 1: Initialization

- Randomly generate users with uniform distribution within each sector, one user per sector. This represents desired and interfering users on a given frequency channel, and assumes that this channel is occupied in all cells at all times.

- Set an initial transmitted power for each user, all users will start simulation with $\mathrm{P}_{\mathrm{T}}=-20 \mathrm{dBw}$. In fact, it doesn't matter to which value we set the initial transmitted power since we will wait till system reaches steady state before collecting data.

- For each user, set up an independent lognormal distributed shadowing. Each subscriber is assigned to the base station with the best SINR (i.e. Macrodiversity). 
Step 2: Simulation over an observation period for specific user

- For each user, after a warming up period, set up Rician fading channel link between the fixed subscriber and the chosen base station. We generate samples of faded signal that is correlated in time. Then, the system is frozen (i.e. we have one snapshot per time). A snapshot represents an interval of time short enough that the channel's path loss can be considered constant.

- Measure the received SINR at the base station, compare it with the preset outer loop threshold $\left(\gamma_{\mathrm{th}}\right)$ and generate up/down power control commands. Note that all subscribers are synchronized together, that is, perform the measurements and the power updates at the same time.

- Perform the power control command, i.e. change the transmitted power of the user in the next sampling period by PC step size $(\Delta)$. If the transmitted power value exceeds the upper bound $(-10 \mathrm{dBw})$ set it to $-10 \mathrm{dBw}$. On the other hand, if it goes below the lower bound $(-50$ $\mathrm{dBw}$ ) then set the updated transmitter value to $-50 \mathrm{dBw}$. In the case of higher PC/snapshot rate, for example 10 , we execute 10 power commands in one snapshot.

- Collect a preset number of fading samples. In order to avoid the border effect, we collect data only for the subscriber in the central cell.

- Calculate the outage probability for the desired user, defined as the fraction of received SINR samples for that user less than the threshold. The system threshold in our simulation is varied from 8 to $12 \mathrm{~dB}$.

Step 3: Repeat the simulation cycle

- Go to step 1 unless number of cycles (subscribers) exceeds preset value chosen as 1000 cycles.

- Calculate the system availability, which is the fraction of desired subscriber positions having less than $1 \%$ outage. 


\subsubsection{Description of the Simulation Software}

MODSIM III has been used to write the computer program designed to simulate the LMCS model. MODSIM III is a general-purpose, modular, block-structured high level programming language, which provides direct support for object-oriented programming and discrete event simulation. The decision to use MODSIM III was mainly because of two features, first of which is the existence of a library module that contains objects and support procedures. The second feature is that all objects can perform actions that elapse simulation time.

An object represents every entity in the model (e.g. subscribers, cells, sectors, carrier frequencies, and channels), and each entity contains a number of fields (variables) used to describe the status of its parameters. The subscriber object carries all the information of the subscriber station such as geographical location, cell number, sector type, distance to other cells, measured SINR.

\subsubsection{Collecting Data}

In order to avoid the impact of the warming up period (initialization), we excluded the results from the initialization stage. We found that excluding 40 samples of SINR were enough before the SINR reaches steady state. For example, the number of excluded samples for the case with power control step size of $0.5 \mathrm{~dB}$ was found to be 40 , since it will need more time to reach steady state compared to the case of power control step of 1 $\mathrm{dB}$ and higher. Therefore, this number of samples is excluded from the outage probability calculation for each user.

Based on a specified SNRR target, we wrote a MATLAB program to calculate the outage probability for that user from the collected samples of SINR. Then we run for another cycle and collect the outage probability for the desired subscriber. After that the system availability can be calculated as the percentage of desired subscribers that have an outage probability less than or equal to $1 \%$. 


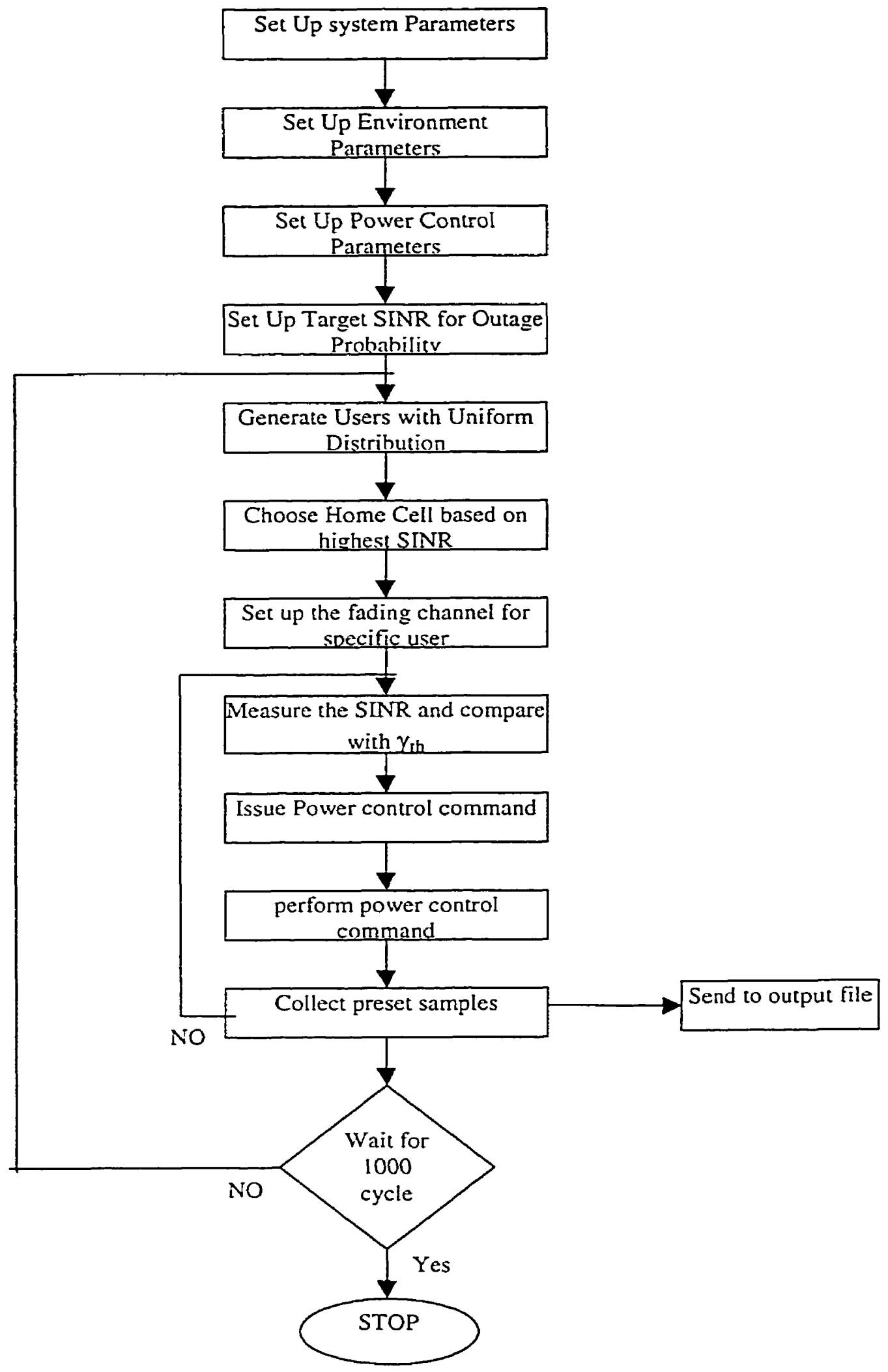

Fig. 4.6 Flow Diagram for the Simulation Program 


\subsection{Issues to be simulated}

In previous chapters, many parameters that can affect the system availability were described. Outage probability is the fraction of time that the signal-to-interference-plus noise ratio is below a certain threshold, due to fading, for a given desired user in a given position. Also, system availability was defined as the percentage of subscribers position having less than $1 \%$ outage. We will investigate the effects of these parameters through simulations. The simulations focus on the implementation of power control techniques in LMCS systems and the impact of different parameters on system availability. The factors, which have been investigated, are listed in Table 4.1. The parameters can be explained as follows:

1. The effect of the propagation environment parameters (such as the propagation exponent, standard deviation of lognormal shadowing, Rician $K$ factor and correlation factor of fading channel ) on system availability is investigated thoroughly by simulation.

2. The effect of fixed step and multistep power control schemes on system performance.

3. Different sets of parameters are investigated such as power control updating rate compared, power control step size, dynamic range of transmitted power and power control threshold.

4. The influence of the macrodiversity technique in system performance is also considered. 


\begin{tabular}{|c|c|c|}
\hline \multicolumn{2}{|l|}{ Issues to be simulated } & Investigation scope \\
\hline \multirow{4}{*}{ Propagation Environment } & $\begin{array}{l}\text { Propagation Exponent } \\
\text { (n) }\end{array}$ & $2-4$ \\
\hline & Rician $\mathrm{K}$ factor & $0-10$ \\
\hline & $\begin{array}{l}\text { Correlation factor } \\
\alpha\end{array}$ & $0,0.2,0.5$ and 0.9 \\
\hline & $\begin{array}{l}\text { Standard deviation of } \\
\text { lognormal shadowing } \\
(\mathrm{dB})\end{array}$ & $6,8,10,12$ \\
\hline \multirow{4}{*}{ Power control } & Step size $(\mathrm{dB})$ & $=0-4$ \\
\hline & $\begin{array}{lll}\text { Power } & \text { control } & \text { per } \\
\text { snapshot } & & \\
\end{array}$ & $0,30,50,100$ \\
\hline & $\begin{array}{l}\text { Outer loop power control } \\
\text { threshold }\left(\gamma_{\mathrm{th}}\right)\end{array}$ & $115,17,19,30$ \\
\hline & $\begin{array}{l}\text { Dynamic Range } \\
\text { (dB) }\end{array}$ & $\begin{array}{l}40 \mathrm{~dB} \\
\text { No upper bound for } \mathrm{P}_{t} \\
\text { No lower bound for } \mathrm{P}_{\mathrm{t}}\end{array}$ \\
\hline Macrodiversity & Selection diversity & $\begin{array}{l}\text { Sellect the base station } \\
\text { thant gives the best SNR }\end{array}$ \\
\hline Outage probability & $\begin{array}{c}\text { system threshold in } \\
\mathrm{dB}\end{array}$ & $8-12$ \\
\hline
\end{tabular}

TABLE 4.1 


\section{Chapter 5 \\ SIMULATION RESULTS}

In this chapter, we will present the simulation results for the LMCS system. The objective of these simulations is to investigate the effect of closed loop power control schemes on system availability, which is related to a number of system parameters. These parameters are related to propagation environment and system design.

Cochannel interference is one of the main issues that need careful study in LMDS systems. This is due to the fact that a LMDS system can reuse the total allocated frequency band in each sector. Transmit power control and macrodiversity are well known techniques in cellular communications to mitigate cochannel interference. These techniques will be considered in our simulation. The simulation results are for the uplink (subscriber to base) only, since power control is most critical for it.

\subsection{Binary SINR-based Power Control}

Transmit power control has been proven in literature to be a very effective technique against near/far problem and shadowing and multipath fading. In chapter 4 , we explained the SINR based and the multistep power control algorithms. In this section, we will investigate the system availability with the implementation of the uplink SINR based power control.

The task of a system designer is to maximize the system availability by implementing different techniques; power control is one of those. The different parameters that describe the effectiveness of power control are power control command update rate, power control step size, transmitted power dynamic range and outer loop threshold $\left(\gamma_{\mathrm{th}}\right)$. The algorithm was explained in detail in chapter 4. 


\subsubsection{Power control command Rate and Step Size}

Fast transmit power control is studied in [1-3] and it is shown that the SINR based power control is able to track multipath fading which gives a lower outage probability. In the fixed broadband wireless system, we will implement this scheme to overcome the multipath fading and improve the overall system availability.

The effect of the power control rate and step size on the system availability is shown in Figure 5.1. The propagation exponent for the desired subscriber $\left(n_{d}\right)$ (i.e. the subscriber in cell 5 , sector 1 ) and the interferer in the opposite sector of the same cell is chosen to be 2 . The interferers coming from other cells will have a propagation exponent $\left(\mathrm{n}_{\mathrm{i}}\right)$ that is chosen to be 4 . We assume a fading channel that is highly correlated with the correlation factor $(\alpha)$ of 0.9 . $K_{i}$ and $K_{d}$ refer to the interferer and the desired user Rician $\mathrm{K}$ factor, respectively.

Figure 5.1 shows the effect of the SINR based power control on system availability. It is shown that a power control per snapshot rate of I will degrade the system availability even more when we increase the step size. Transmitting with a PC/snapshot of 10 will slightly improve the system availability with a step size of 2-3 dB, achieving 0.714 system availability. Further increase of the power control step size will degrade the system performance. This is due to the fact that when the received SINR is slightly larger than the desired threshold, the base station issues a down command. Therefore, the SINR will degrade and the outage probability becomes worse, for example with a step size of 4 the system availability is 0.554 .

The higher the power control command per snapshot rate the better the system performance will be. This can be observed for the cases of PC/snapshots of 30, 50 and 100. Further increase of the power command rate per snapshot is not beneficial for the system availability especially in the case of higher power control step size. We should also take into consideration not to send too many power commands that would consume too much of the available bandwidth. 
We can conclude from this figure, that in order to track multipath fading, we need to send power control commands as fast as we can in order to compensate for multipath fading.

In propagation environments where the fading rate of the channel is slow we can reduce the power control command rate. On the other hand, for a higher fading rate we need a higher rate of power control commands. A trade off between power command rate and available resources must be taken into consideration. Since sending more power command than the required level will reduce the channel resources while they may be used for other vital system transmissions.

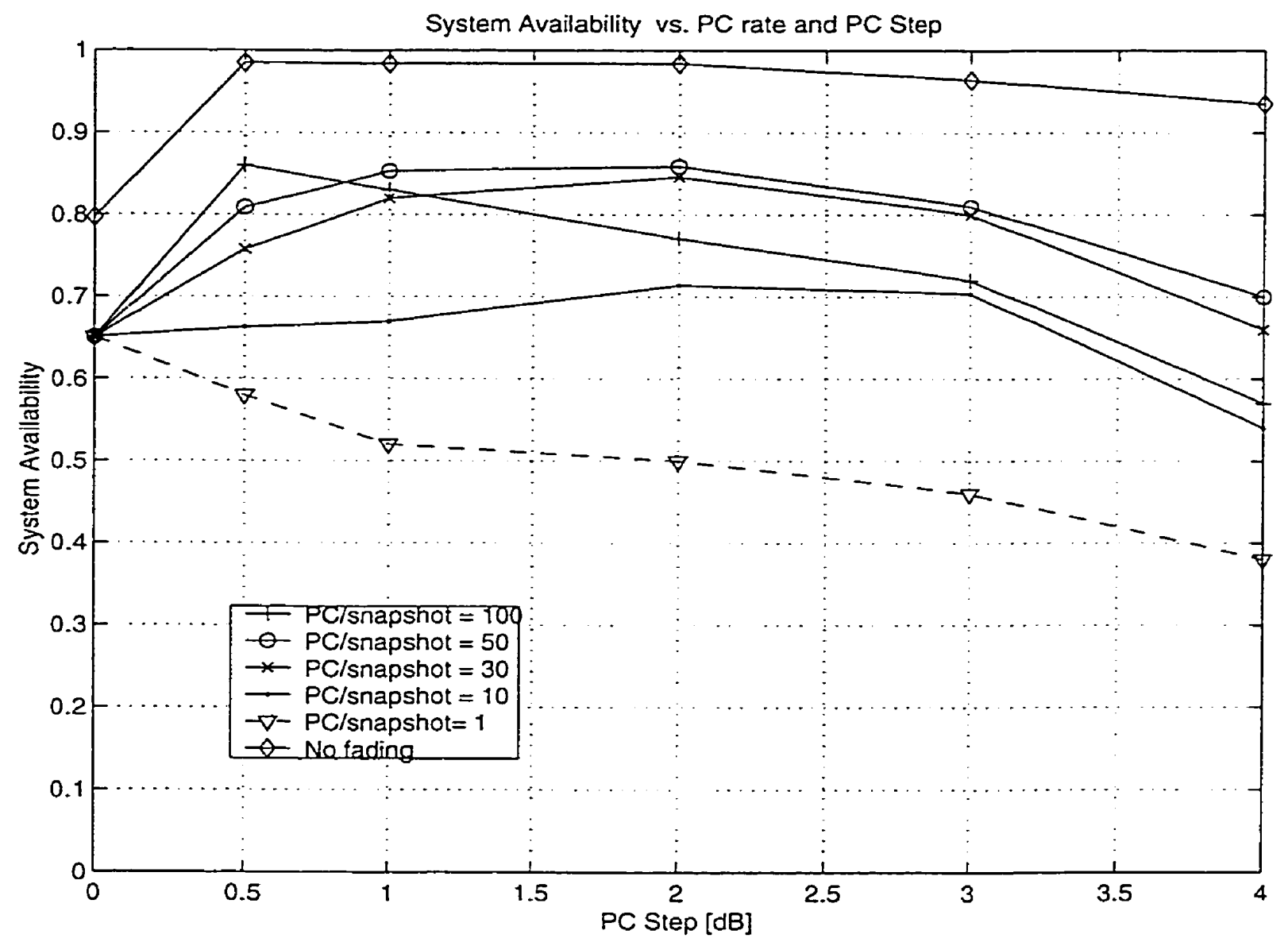

Figure 5.1 The effect of power command rate and step size on system availability, with $n_{d}=2, n_{i}=4$, correlation factor $\alpha=0.9, K_{i}=4, K_{d}=10$, dynamic range $=40 \mathrm{dBw}$. 
Another important parameter that affects the system availability is the power control step size. We will try to give the optimum power control step size for the SINR based power control scheme that provides the best system availability. The power control step size should be carefully selected, since a power control with small step size needs more time to compensate deep fades of the signal. On the other hand, sending power commands with a higher step size may disturb the system when the fading signal improves.

System stability is very important as we mentioned in chapter 4, so we excluded the received SINR data during the transit response. Since the system will take some time before it reaches steady state. The number of excluded samples depends on the power control step size, since higher step sizes will reach the steady state before small step sizes.

In this simulation, we will suggest an optimum step size for the SINR based power control for each of the power control update rates. Figure 5.I shows the effect of different step size on system availability. It is observed from Figure 5.1 that a power control per snapshot of 10 with a power control step size of $2 \mathrm{~dB}$ slightly improves the system availability among the other values of step size.

Increasing the $\mathrm{PC} /$ snapshot ratio to 30 or even 50 , indicates that the optimum power control step size is $2 \mathrm{~dB}$. Furthermore, a PC/snapshot ratio of 100 with power control step of $0.5 \mathrm{~dB}$ shows the best system availability of 0.86 . We also noticed that if we send power commands with a higher step size for example $4 \mathrm{~dB}$, the system availability would degrade. This can be explained as follows: in the case of high power control commands rate, the higher step size increases the interference, which consequently causes the higher outage probability. Therefore, for higher power control update rates smaller step size is more desirable.

In the case of no power control (i.e. power control step size of $0 \mathrm{~dB}$ ), it is shown that system availability in all cases started from the same point and then diverged. For the case of no fading and no power control, the system achieves a higher availability, since 
there is no multipath fading and the subscriber will face only shadowing. Hence, the system availability is improved. Note that the upper curve in Figure 5.1 represent the system availability solely due to the shadowing and path loss.

The previous discussion of the results indicates that there is a trade-off between the optimum power control command rate and the optimum step size. In order to achieve the best system availability we need to take into consideration both parameters.

\subsubsection{Effect of Dynamic Range}

The effect of the transmitted power dynamic range on system availability is shown in Figure 5.2. It is observed from this figure that the value of the upper bound of the transmitted power is fairly important. This is due to the fact that, no upper bound for $\mathrm{P}_{\mathrm{L}}$ will allow subscribers with high outage probability to increase their transmitted power in order to satisfy the SINR requirement by the base station, which in turn causes severe interference to other subscribers. At the same time, other subscribers will increase their transmitted power to keep the quality of signal at a certain acceptable level and this positive feedback will worsen the overall performance.

In Figure 5.2, it is observed that removing the lower bound restriction on the subscribertransmitted power will give a slight further improvement in the system availability. Since no lower bound condition on the transmitted power allow subscribers to reduce their transmitted power if the service requirement by the hub is satisfied, this reduction in transmitted power will reduce the interference to other subscribers and enhance the overall system performance. On the other hand, if the upper bound condition on the transmitted power is released, the system availability will be slightly degraded to 0.79 at an outage probability of $1 \%$. Setting the transmitted power dynamic range to $40 \mathrm{dBw}$ gives a system availability, which is approximately equivalent to the case of no lower bound. 
It is the task of the system designer to assure that the transmitter has the suitable dynamic range to compensate for the distance path loss, rain and multipath fading.

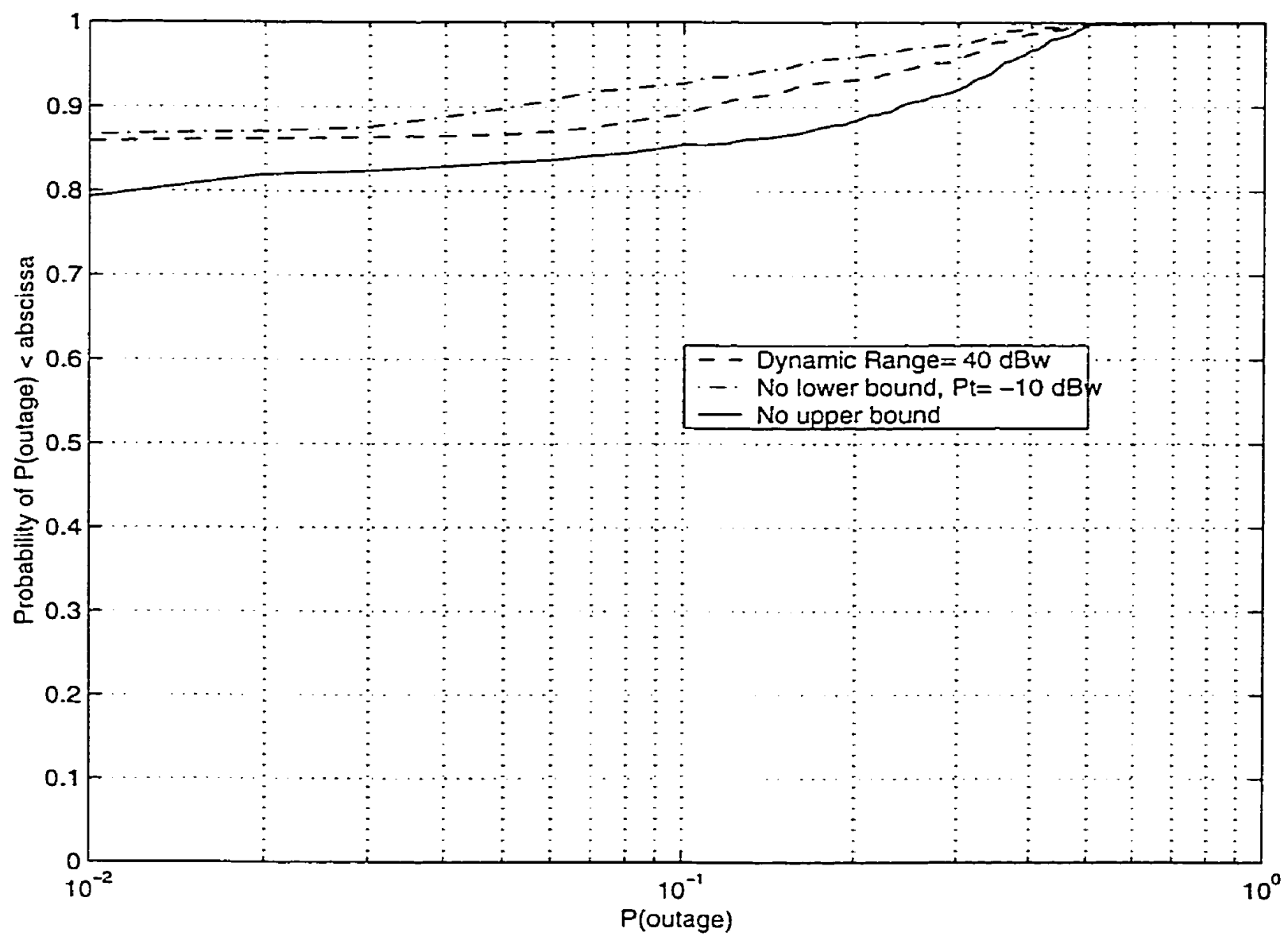

Figure 5.2 Effect of transmit power dynamic range on system availability, $\mathrm{PC} / \mathrm{snapshot}=\mathbf{5 0}$, power control step $=2 \mathrm{~dB}, K_{i}=4$ and $K_{d}=10$.

\subsubsection{Outer loop threshold}

The effect of the outer loop threshold $\left(\gamma_{\mathrm{th}}\right)$ on system availability is simulated and shown by Figure 5.3. The outer loop threshold can be defined as the required SINR value to satisfy a desired error rate. We assume a fixed value $\left(\gamma_{t h}\right)$ for all subscribers. The performance is highly dependent on the value of $\gamma_{\mathrm{th}}$. This value can be adjusted according to the frame error rate (FER). If the FER is satisfactory the $\gamma_{\mathrm{th}}$ can be reduced, otherwise it is increased. 
Fig. 5.3 shows that a system availability of 0.714 can be achieved with the outer loop threshold $\gamma_{\mathrm{th}}=15 \mathrm{~dB}$. Increasing the threshold to $17 \mathrm{~dB}$ gives system availability of 0.83 . Further improvement on the availability can be achieved with the power control threshold $=19 \mathrm{~dB}$ that is 0.872 . More subscribers can achieve the required outer loop thres hold, which leads to a lower outage probability and improve the system availability. Notice the diminishing return that happens when we set the threshold to a high value of $30 \mathrm{~dB}$. The system availability will be degraded, because at such high threshold value the subscribers will be forced to increase their transmitted power in order to satisfy the required s.ignal quality at the hub. This causes more interference to the subscribers with a good quality and will not benefit the subscribers with a poor quality. In order to avoid this we caen set an upper bound on the transmitted power in addition to choosing a reasonable valure for the outer loop threshold.

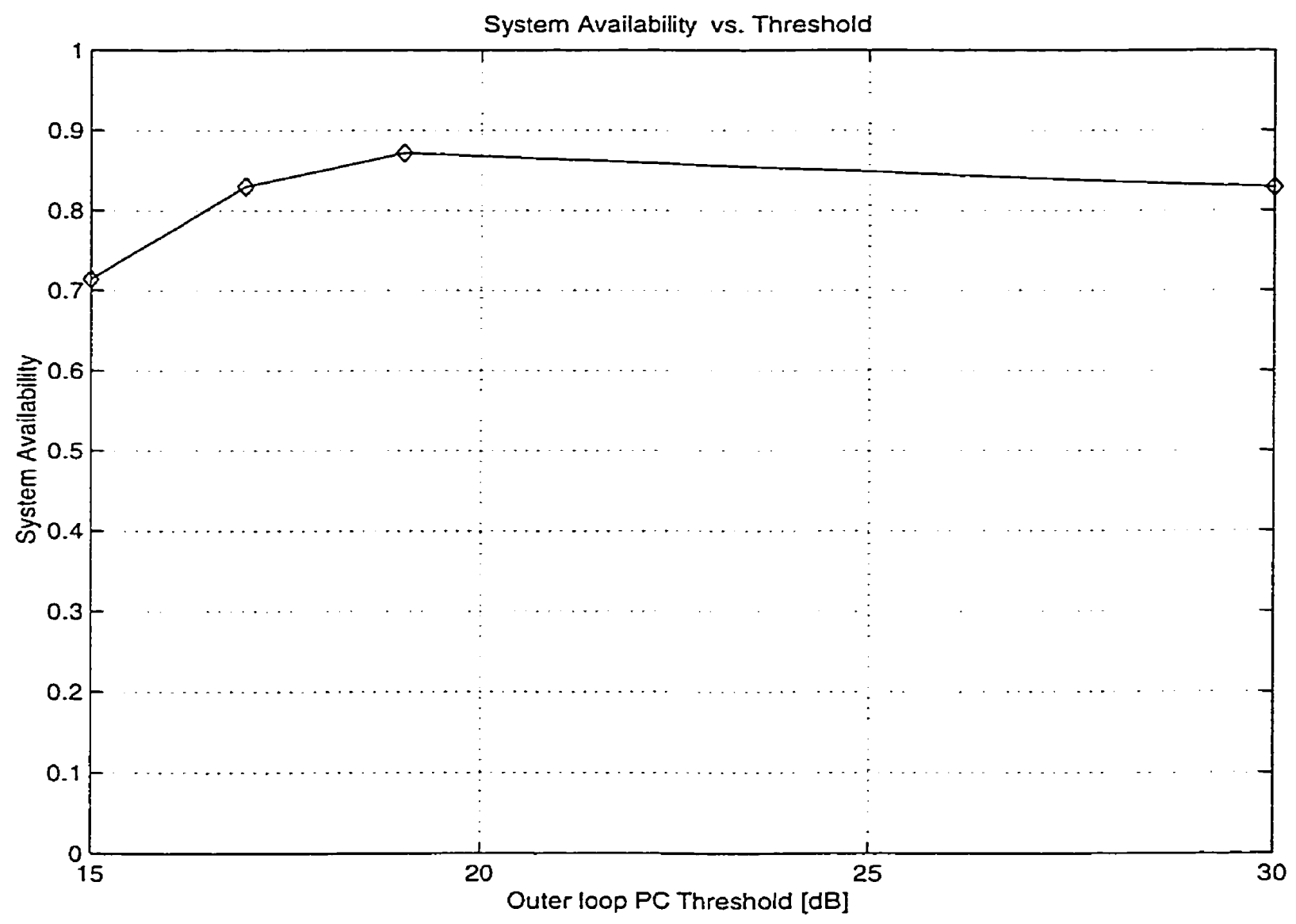

Figure 5.3 Effect of outer loop threshold $\gamma_{t h}$ on the system availability, PC/snapshot=10, PC step $=2$ $\mathrm{dB},\left(\mathrm{n}_{\mathrm{d}}, \mathrm{n}_{\mathrm{l}}\right)=(2,4)$, modem threshold $10 \mathrm{~dB}, K_{i}=4$ and $K_{d}=10$. 


\subsubsection{Modem Threshold}

The modem threshold is defined as the minimum required SINR to achieve a certain BER. At this given value the outage probability for a given subscriber can be calculated as the probability that the received SINR is less than the modem threshold or target SINR.

For 4-QAM modulation, a bit error rate (BER) of $10^{-3}$ requires a target SINR of $10 \mathrm{~dB}$, which might be suitable for voice communications. But broadband service system requirements might need a lower $B E R$, which can be satisfied using the same target SINR, but with the implementation of error control coding.

Figure 5.4 shows the results of a simulation of the effect of the modem threshold on the system availability. It is observed that at a higher ratio of PC/snapshot (i.e. PC/snapshot $=50$ ) a modem threshold of $8 \mathrm{~dB}$ that is $4 \mathrm{~dB}$ lower than the outer loop PC threshold shows a system availability of 0.859 . On the other hand, at PC/snapshot of 10 , the modem threshold of $8 \mathrm{~dB}$ shows a system availability of 0.849

In digital communications, it might be advantageous to employ some coding schemes that will lower the modem threshold, and therefore enhance the outage probability of the system. The value of the required modem threshold can be traded with a better BER. 


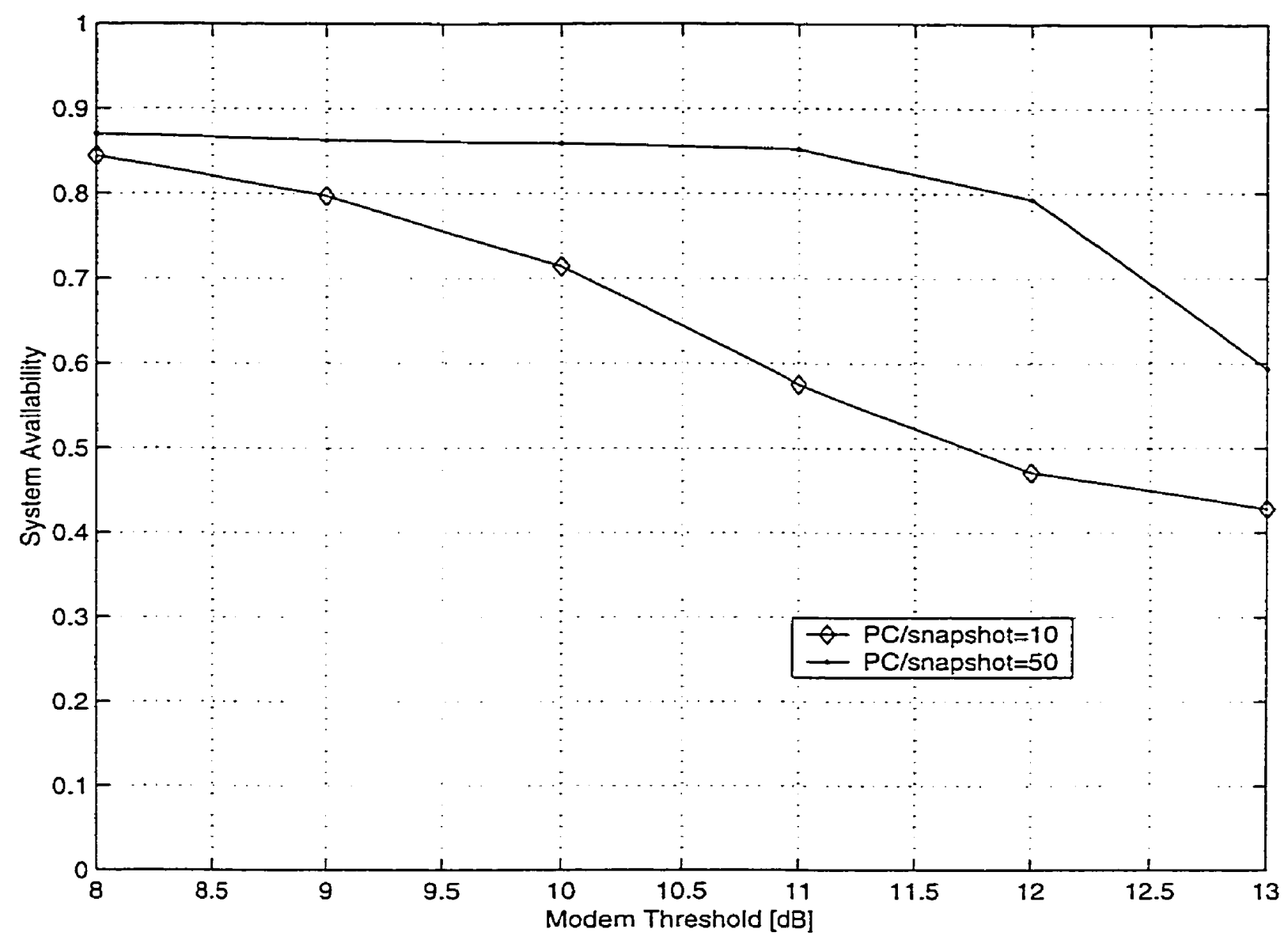

Figure 5.4 System availability as function of modem threshold for PC step $\Delta=2 \mathrm{~dB},\left(\mathrm{n}_{\mathrm{d}}, \mathrm{n}_{\mathrm{I}}\right)=(2,4)$, outer loop $\mathrm{PC}$ threshold $=15 \mathrm{~dB}, K_{i}=4$ and $K_{d}=10$.

\subsection{Effects of Propagation Environment}

We study the effect of the propagation environment parameters on the system availability for cellular fixed broadband wireless systems such as LMCS. As we described in chapter 4 , these parameters are the propagation exponent, the standard deviation of lognormal shadowing, the Rician $K$ factor for the desired subscriber and interferers and time correlation factor of fading channel. In the next subsection, we will discuss the effect of these parameters on system performance in conjunction with uplink SLNR based power control algorithm. 


\subsubsection{The Effect of Propagation Exponent}

The effect of propagation exponent $n$ on system availability for LMCS is simulated and the results are shown in Figure 5.5 and Figure 5.6. In this simulation we fixed either the interferers or the desired subscriber propagation exponent and changed the others propagation exponent for values of 2,3 and 4 .

It is observed in Figure 5.5 that a small propagation exponent for the desired subscriber yields a lower outage probability and thus a significant increase in system availability, e.g. using power control per snapshot of 10 and PC step size of $2 \mathrm{~dB}$, the system availability of 0.714 can be achieved with desired subscriber propagation exponent ( $n_{d}$ ) equal to 2. If the desired propagation exponent is increased to 3 and 4 , system availability is reduced to 0.461 and 0.312 , respectively.

The reason of reduced system availability is as follows: In the case of small propagation exponent, the received signal is less attenuated in comparison to the case of high propagation exponent. So, the received SINR for the desired subscriber is better compared to the case when it has a higher propagation exponent. The same conclusion can be drawn from Figure 5.5 for the case of a higher PC/snapshot rate of 50 .

The results for the case of the desired subscriber propagation exponent $\left(n_{d}=2\right)$ shows that the system availability is improved from 0.714 and 0.859 for PC/snapshot rate of 10 and 50, respectively. Refer to the case of no fading in Figure 5.1; we observed that for the same $n_{d}$ and a step size of $2 \mathrm{~dB}$, the achieved system availability is improved to 0.984 . We conclude from this that the effect of multipath fading is significant on the system availability.

In Figure 5.6, we show the system availability as function of propagation exponent of interferers $\left(n_{i}\right)$. Here, we fix the desired subscriber propagation exponent to 2 . It is observed that the system availability increases significantly from 0.47 to 0.714 when the propagation exponent of interferers increases from 2 to 4 . These results are for the case of 
$\mathrm{PC} /$ snapshot $=10$. Since higher values of propagation exponent for out of cell interferers leads to more attenuation to their signal which gives a higher SINR at the hub. Thus, the outage probability for the desired subscriber would benefit from the high attenuation of the interfering signals, and will be reduced, which enhances the system availability. The same conclusion can be drawn for the case of PC/snapshot $=50$, where the system availability increases from 0.58 to 0.859 when the interferer's propagation exponent are increased from 2 to 4.

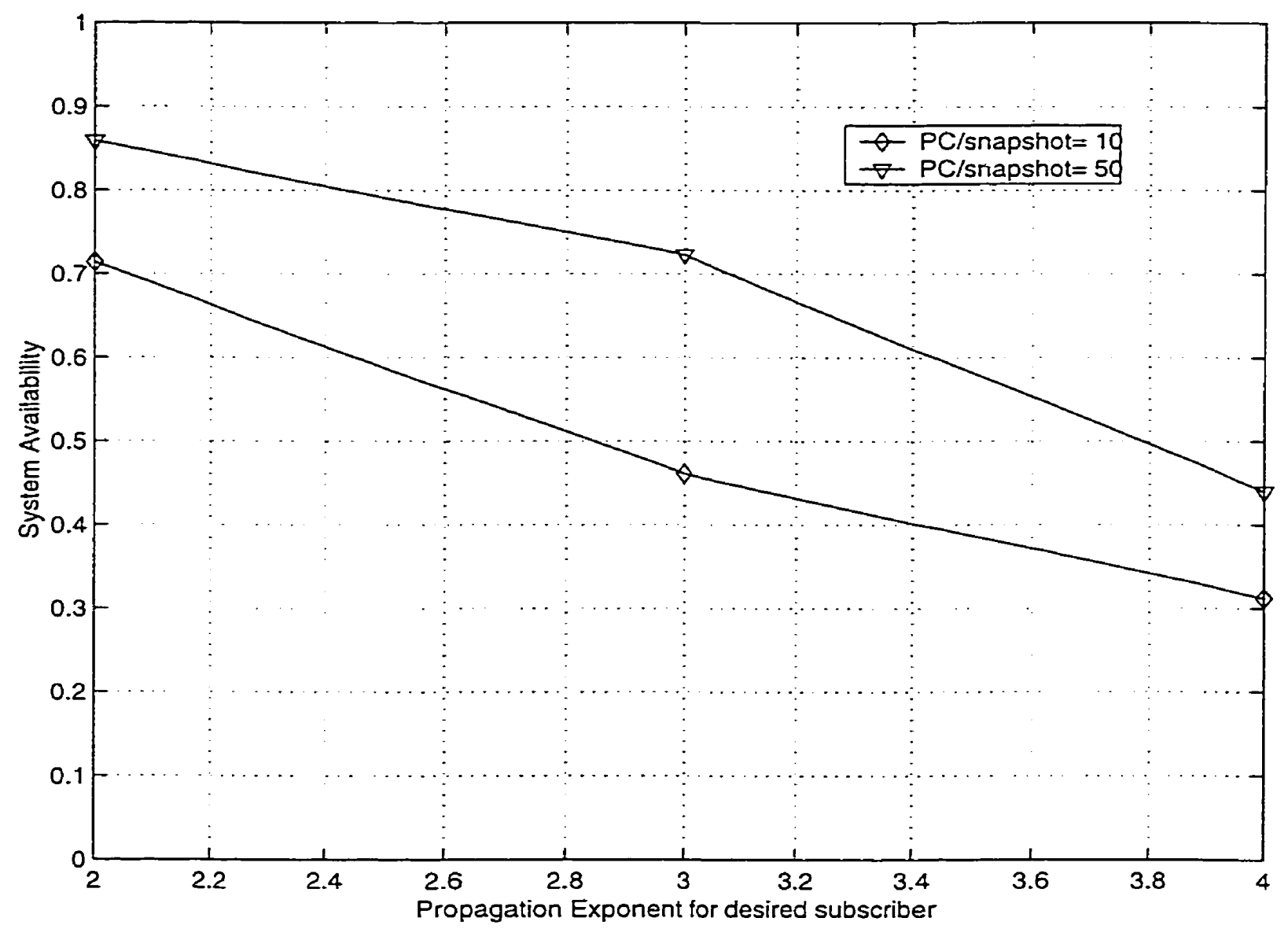

Figure 5.5 System availability versus propagation exponent of desired subscriber $\left(n_{d}\right), n_{i}=4$. $P C /$ snapshot $=10, \mathrm{PC}$ step $\Delta=2 \mathrm{~dB}$, correlation factor $\alpha=0.9, K_{i}=4$ and $K_{d}=10$. 


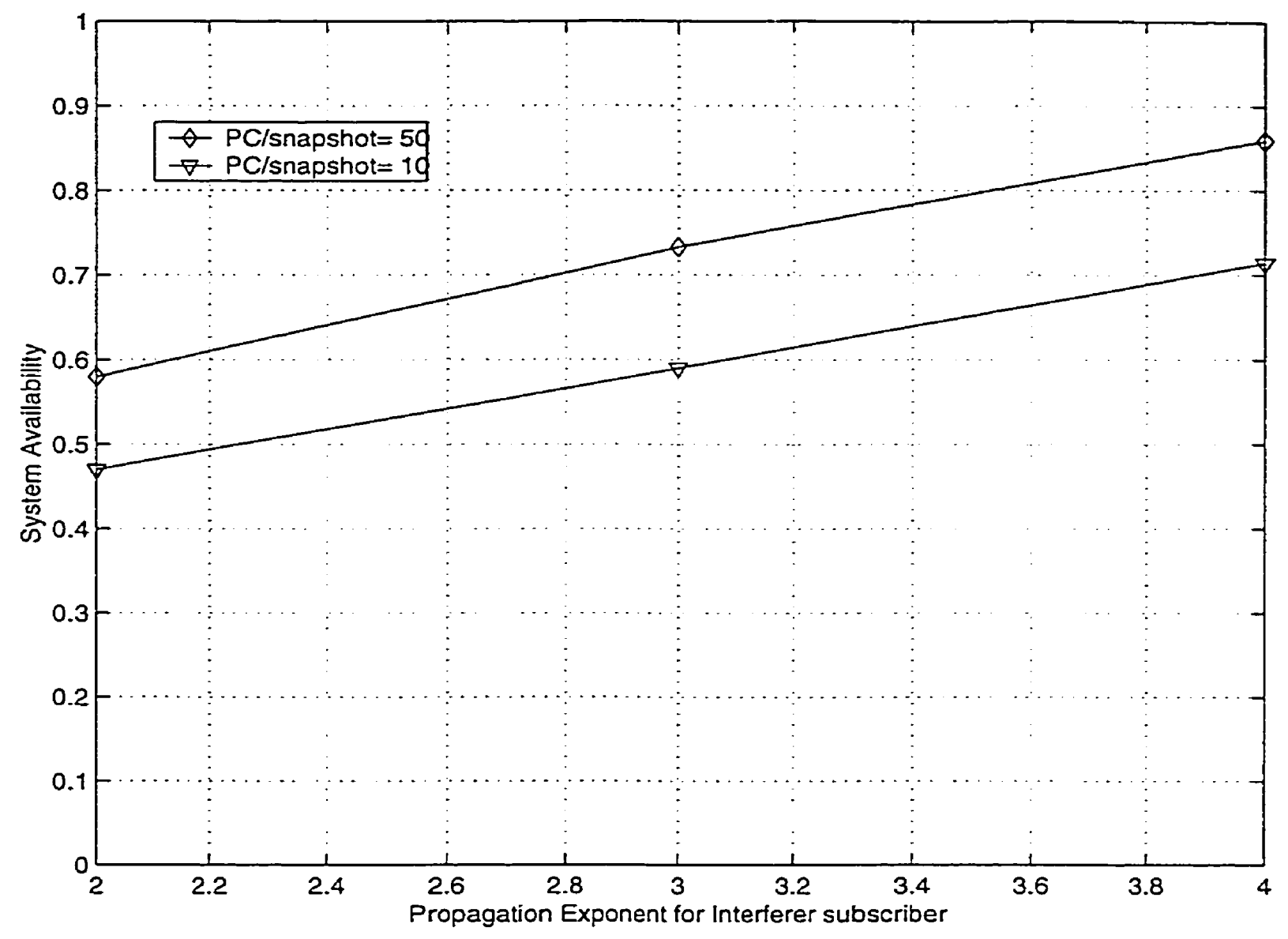

Figure 5.6 System availability versus propagation exponent of interferers subscriber $\left(n_{i}\right), n_{d}=2, P C$ step $\Delta=2 \mathrm{~dB}$, and correlation factor $\alpha=0.9, K_{i}=4$ and $K_{d}=10$.

\subsubsection{Standard Deviation of Shadowing}

The drastic effect of lognormal shadowing standard deviation on the system availability is illustrated in Figure 5.7. The system availability for PC/snapshot $=10$ decreases from 0.9 to 0.52 as the lognormal shadowing standard deviation $\sigma$ increases from $6 \mathrm{~dB}$ to 12 $\mathrm{dB}$. At higher power control command to snapshot ratio such as 50 , the degradation in the system availability is lower than that for $\mathrm{PC} /$ snapshot $=10$. It can be noticed that standard deviation of $12 \mathrm{~dB}$ (at PC/snapshot $=50$ ) almost gives the same system availability for a shadowing standard deviation $\sigma=8 \mathrm{~dB}$ (for a $\mathrm{PC} /$ snapshot rate $=10$ ). 
This is because a higher standard deviation for lognormal shadowing means more variations in the signal level. In order to provide a good quality of service and high coverage in a downtown area with heavy shadowing effect, smaller cells should be used that gives a higher chance for a LOS path between the subscriber and the hub.

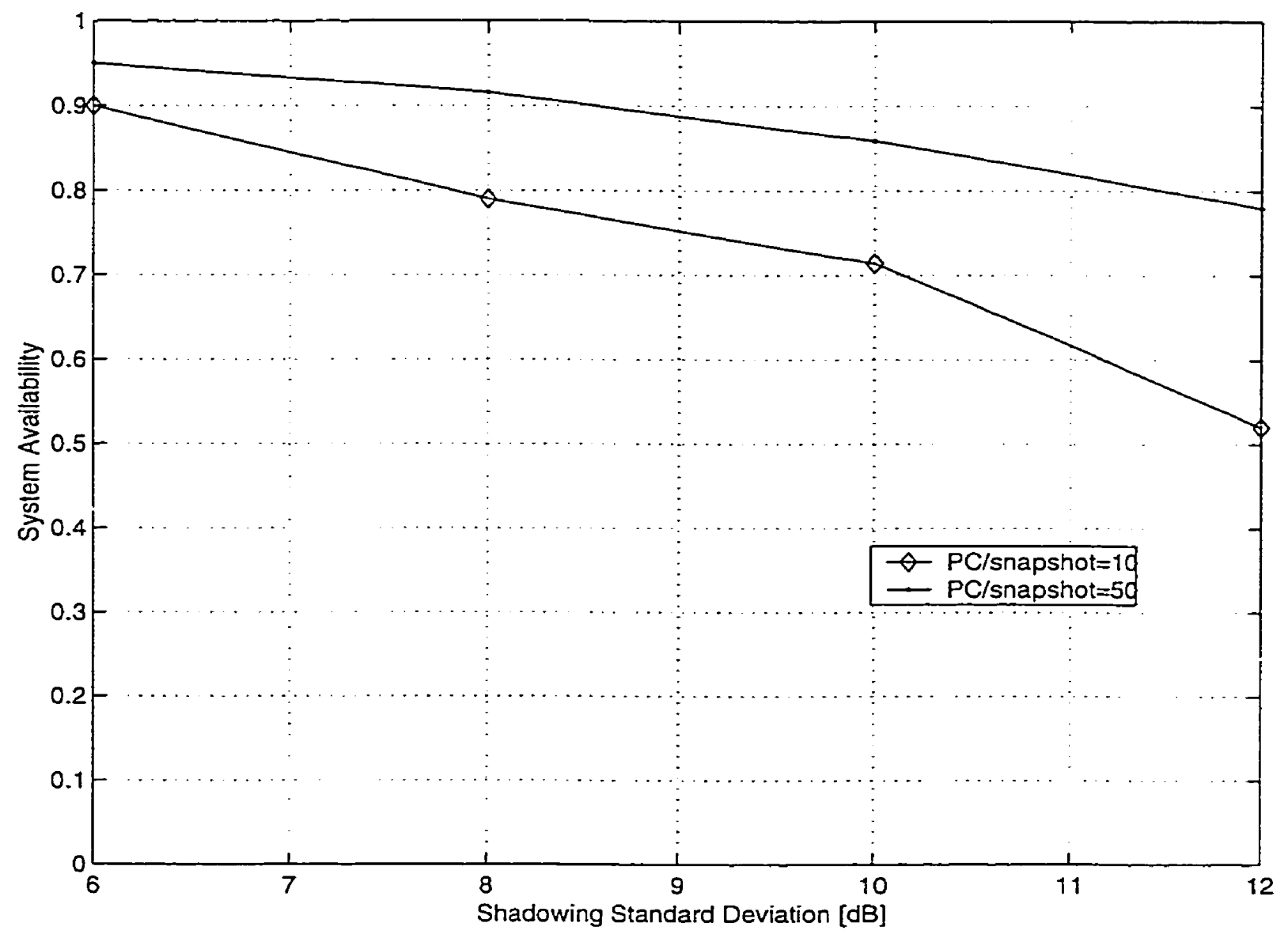

Figure 5.7 System availability versus standard deviation of lognormal shadowing, PC step $\Delta=2 \mathrm{~dB}$, correlation factor $\alpha=0.9, \mathrm{n}_{\mathrm{d}}=2, \mathrm{n}_{\mathrm{i}}=4 ., K_{i}=4$ and $K_{d}=10$.

\subsubsection{Effect of Rician $\mathrm{K}$ factor}

As we previously discussed in chapter 3, Rician fading can be represented by the Rician $K$ factor which is the ratio of the LOS power component to the power of the scatter component. Higher values of $\mathrm{K}$ mean a stronger LOS path between the hub and the subscriber. 
Adequate coverage in fixed broadband wireless system requires a direct LOS between the hub and subscriber.

In this section, we simulate the effect of Rician $K$ factor for the desired subscriber on system availability. We assume that the interferes have a Rician $K$ factor of 4 .

Figure 5.8 show that higher values of Rician $K$ factor for the desired subscriber yields a better system availability. At PC/snapshot $=10$, if we increase the $K_{d}$ factor from zero (i.e. Rayleigh fading) to 10 , it will significantly increase the system availability from 0.169 to 0.714 , respectively. This is due to the fact that a better LOS link between the hub and the desired subscriber improves the received SINR and lowers the outage probability. Furthermore, increasing the power control command rate per snapshot to 50 improves the system availability even more. For example, at $K_{d}=10$ and $\mathrm{PC} /$ snapshot $=10$, the achieved system availability is 0.714 while for $\mathrm{PC} /$ snapshot $=50$, it will increase to 0.859 . 


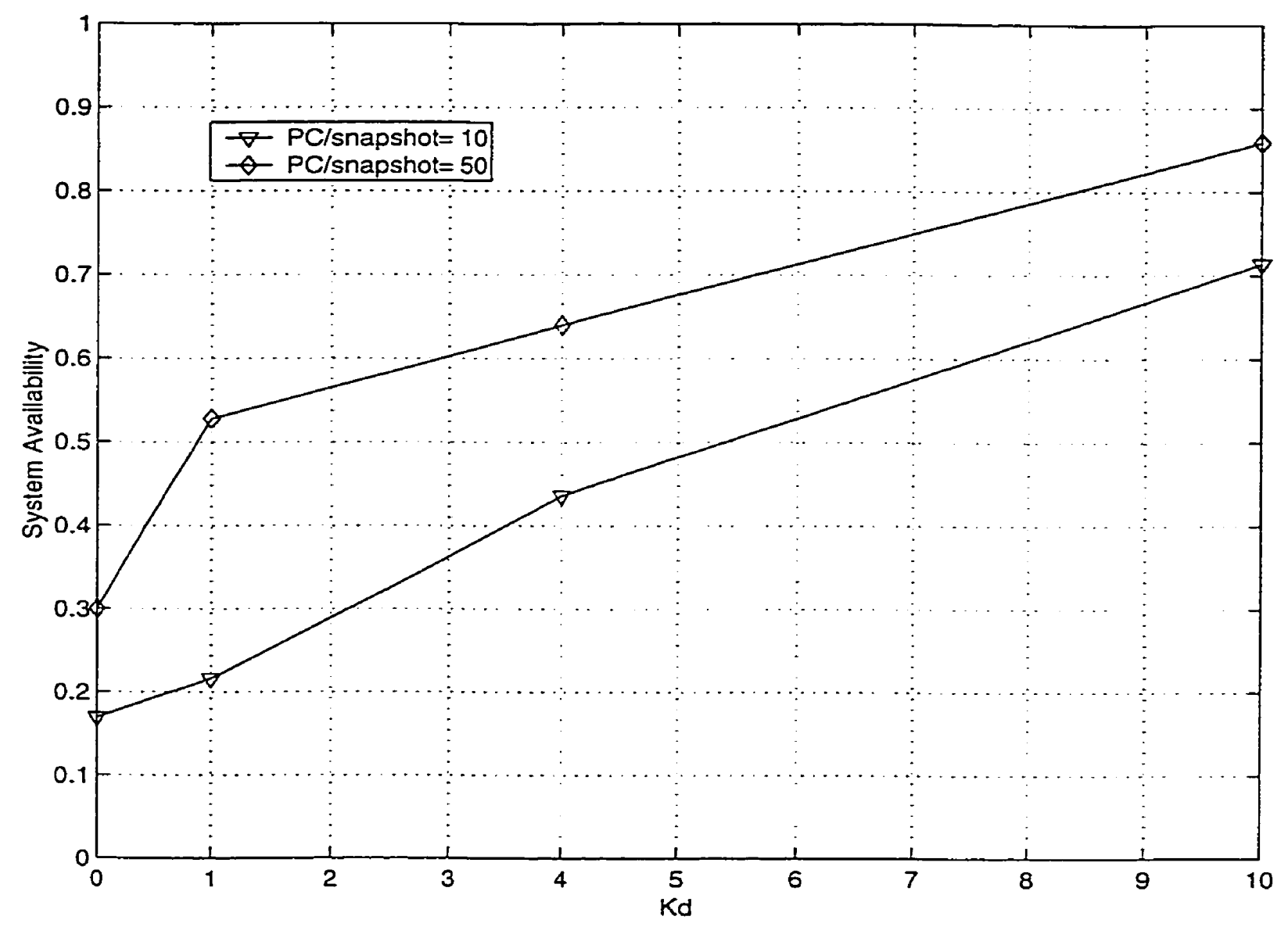

Figure 5.8 The effect of desired subscriber Rician $\mathrm{K}$ factor on system availability, PC step $=2 \mathrm{~dB},\left(\mathbf{n}_{\mathrm{d}}\right.$, $\left.n_{i}\right)=(2,4)$, and correlation factor $\alpha=0.9$

\subsubsection{Effect of Correlation factor}

A fading signal that is correlated in time may be tracked more accurately compared to the case of independent fading channel. The time correlation is implemented using a firstorder autoregressive process, by passing the Gaussian r.v's that generate the Rician r.v through a low pass filter with a correlation factor $\alpha$, as explained in chapter 4 .

The Rician fading has been considered for both the desired subscriber channel link and interferers links. Figure 5.9 shows the effect of the correlation factor on the system availability for the LMCS system using the SINR based power control with PC step $\Delta=2$ $\mathrm{dB}$ and propagation exponent $\left(\mathrm{n}_{\mathrm{d}}, \mathrm{n}_{\mathrm{i}}\right)$ of 2 and 4 . 
Figure 5.9 shows a significant increase in system availability, as the fading channel becomes highly correlated (i.e. 0.9); the system availability is 0.714 at $\mathrm{PC} /$ snapshot $=10$. Reducing the correlation factor to 0.2 will degrade the availability to 0.64 , independent fading channel (i.e. correlation factor of zero) shows a system availability of 0.4 . This essentially means that for the propagation channels with low correlation factor the variations in the channel is more frequent and a low power control update rate is not able to track the channel changes. Therefore, the system availability is decreased.

In the case of a highly correlated fading signal, the signal variations are small, and power control will be able to track the multipath fading, whereas for the case of low correlation (i.e. almost independent) the power control update rate must be high enough to be able to track the changes in the channel.

If we have the resources to send a higher number of power control commands per snapshot we can observe that PC/snapshot of 50 can track the multipath fading even for a lower correlation factor of 0.2 . 


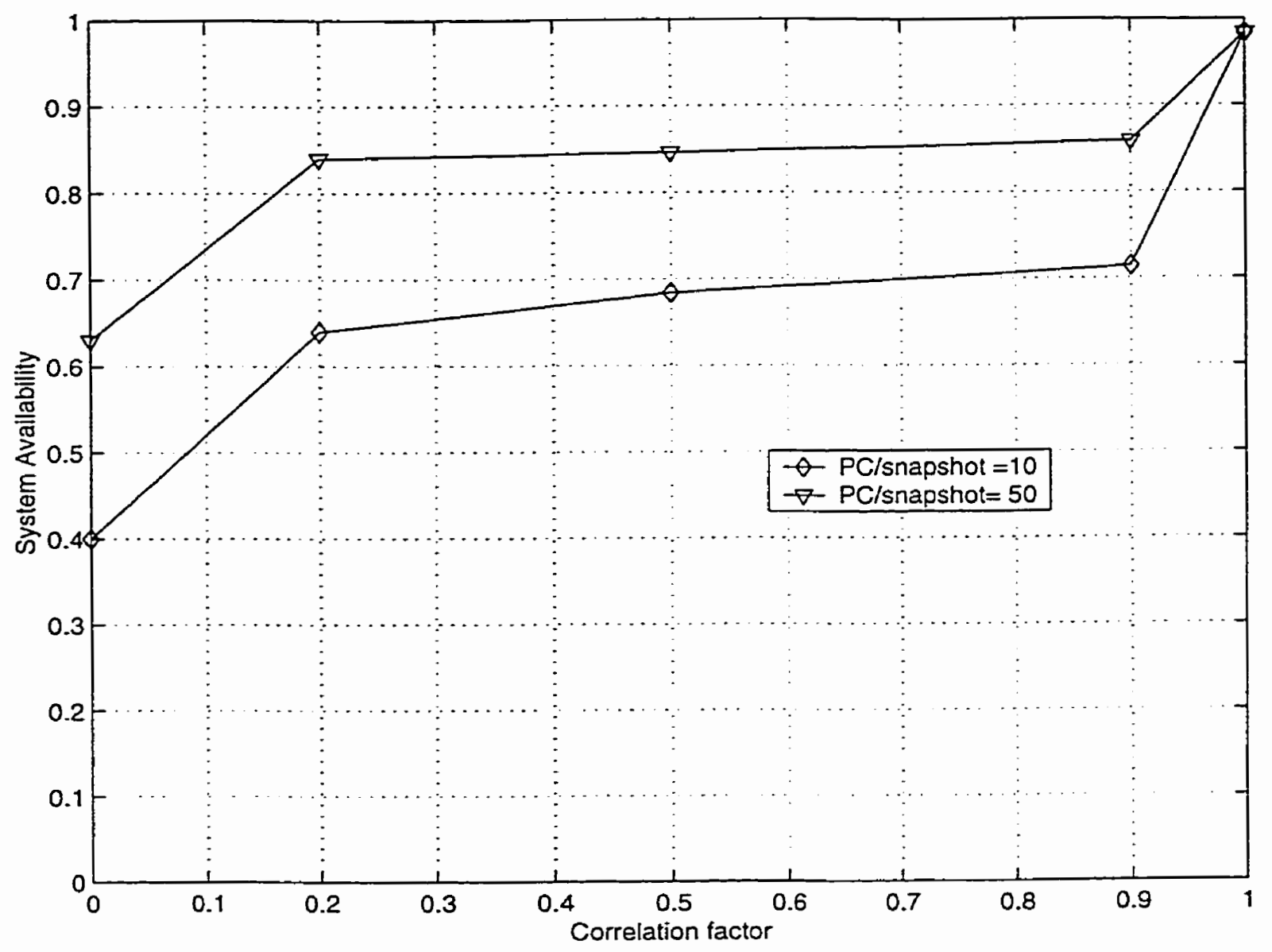

Figure 5.9 System availability vs. correlation factor $\alpha, \Delta=2 \mathrm{~dB},\left(\mathrm{n}_{\mathrm{d}}, \mathrm{n}_{\mathrm{i}}\right)=(2,4), K_{i}=4$ and $K_{d}=10$. 


\subsection{Multistep SINR-based Power control}

In chapter 4, we described the multistep SINR-based power control scheme. The effect of system parameters such as power control quantization mode- $n$, optimum step size and the optimum power control threshold are investigated in this section.

\subsubsection{Effect of Quantization Mode}

The error defined as the difference between the received SINR and a desired level is fed into the power command decision block for the multistep power control scheme. This block sends a power control command denoted by $\mathrm{cmd}$, which is the quantization value of the error, to the subscriber via a feedback channel.

Figure 5.10 shows the system availability as function of different power control mode. It is clear that a lower mode value in this case mode $1(\{-1,0,1\}$ steps $)$ will give the best system availability, since transmitting with a higher power steps will disturb the system performance and degrade the availability for the subscribers. Increasing the power control mode from 1 to 3 will degrade the system availability from 0.793 to 0.544 . Furthermore, mode 3 has to send 3 bits for each power control command that will limits the available bandwidth. Thus we recommend using multistep power control with mode 1.

There is a relation between quantization level $\mathrm{L}$ and the number of power control command bits $n$. It can be described by $n=\log _{2} L$. For example, mode-1 will send a 2 bits/command. Choosing a higher power control mode means transmitting more bits per power control command, which consumes the available bandwidth. It is shown in [3] that multistep power control scheme with higher mode improves the outage performance compared to lower mode. Setting the power control mode beyond a certain value will worsen the system outage. 


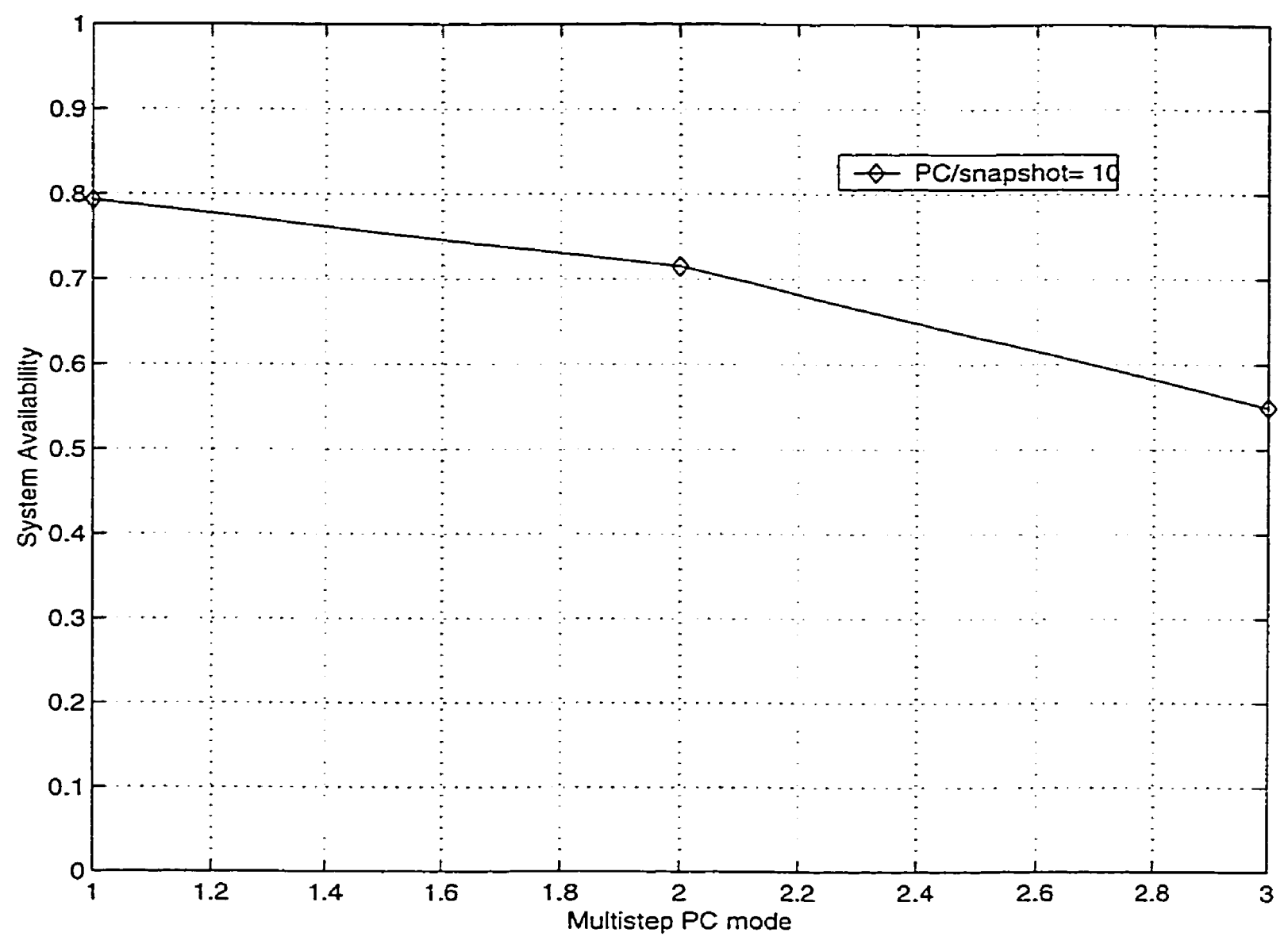

Figure $\mathbf{5 . 1 0}$ system availability versus mode-n multistep power control, with $\mathrm{PC} /$ snapshot ratio = 10, power control step $=1 \mathrm{~dB},\left(\mathrm{n}_{\mathrm{d}}, \mathrm{n}_{\mathrm{D}}\right)=(2,4), K_{i}=4$ and $K_{d}=10$.

\subsubsection{Optimum step size}

The system availability as function of the initial power control step size for multistep power control scheme with mode-1 is shown in Figure 5.11. It is obvious for this choice of parameters that a step size of $1 \mathrm{~dB}$ gives the best performance compared to other values of power control step. We further observe that if power control step $\Delta$ is either too small or too large, the system availability decreases. Therefore, there exists an optimum power control step size, which is $1 \mathrm{~dB}$. 


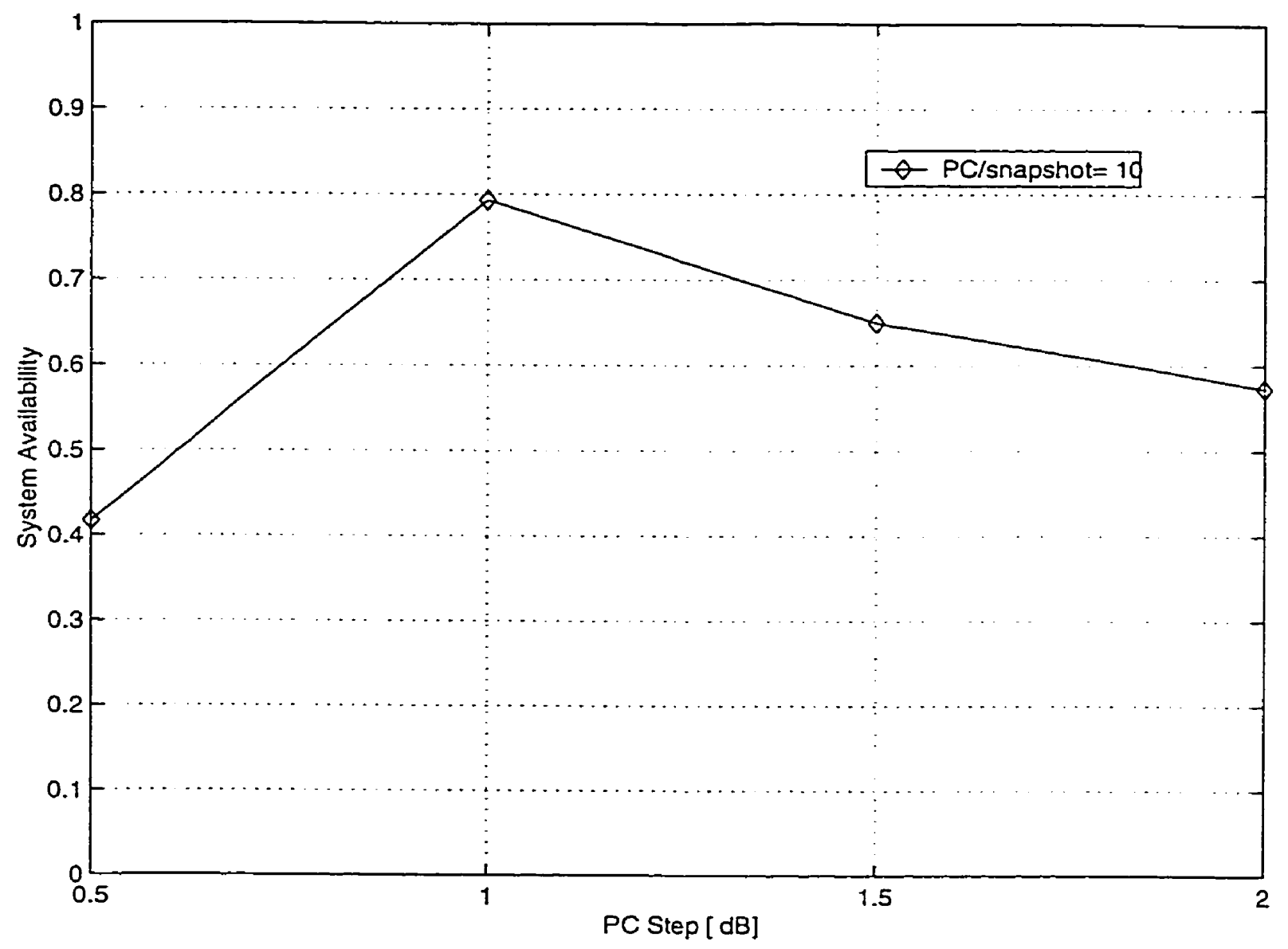

Figure 5.11 System availability versus power control step size for multistep power control of mode-1, correlation factor $=0.9, K_{i}=4$ and $K_{d}=10$.

\subsection{Binary SINR based vs. Multi-step SINR-based Power control}

In chapter 4 of this thesis, we describe two schemes that are the binary SINR-based power control and the multistep SINR-based power control. Furthermore, in previous sections of this chapter, we investigate the system availability for both schemes. Here, we will compare both schemes in terms of system availability.

In order to make a fair comparison between both schemes, all the assumptions for both schemes should be the same. For example, we assume that the binary SINR-based power control scheme has a power control update rate of 20 in one snapshot and the desired and interferers subscribers will have a propagation exponent of 2 and 4, respectively. 
Furthermore, we consider a multistep power control scheme with mode $1(-1,0$ and +1$)$ and power control per snapshot rate of 10 . Since each command consists of 2 bits we will have a total of $20 \mathrm{bit} / \mathrm{snapshot,}$ which is equivalent to the binary SINR based power control rate.

It is shown in Figure 5.12 that the system availability for the binary SINR based power control is 0.77 and for the multistep SINR-based power control is 0.793 . It can be concluded that the multistep SINR-based power control scheme gives a better availability than the binary SINR based power control although the difference is not significant. Since for the same power control per snapshot we have to send more bits per snapshot, which consumes more of the available bandwidth, it is recommended to use the binary SINR based power control scheme instead of the multistep SINR-based power control.

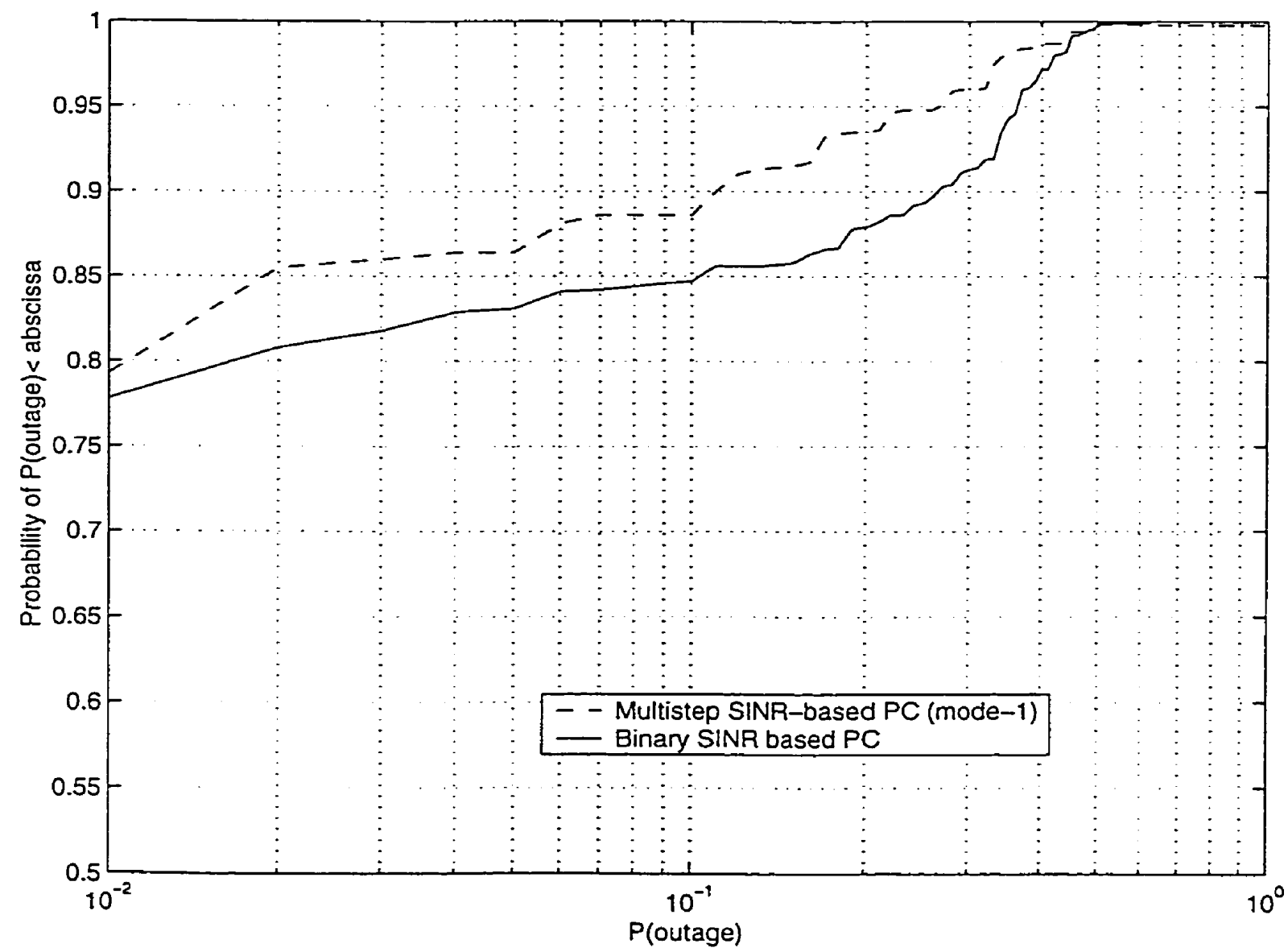

Figure 5.12 Binary SINR based vs. multistep SINR-based $P C$, for $P C /$ snapshot $=20, P C$ step $=1 \mathrm{~dB}$ and $\left(\mathbf{n}_{\mathrm{d}}, \mathrm{n}_{\mathrm{i}}\right)=(2,4)$. 


\subsection{Effect of Macrodiversity}

The effect of power control techniques on the performance of fixed subscribers in fixed broadband wireless systems (LMCS) has been investigated in previous sections of this chapter. Another important technique that is used to mitigate the multipath fading and the large-scale fading (shadowing) in mobile communications is macrodiversity.

Site macrodiversity allows a subscriber to select to communicate with the base station in the system based on the best-received SINR. The study in [15] shows that using macrodiversity with overlapping cells, can significantly enhance the outage performance and increase the system coverage. The results indicate that $20 \%$ coverage improvement is possible when using 4 hubs instead of 1 in typical suburban area.

In our simulation, we assumed that there is no overlap between cells. The results in Figure 5.13 show that employing macrodiversity enhances system availability. Using power control will significantly improve the system performance, with a smaller effect on system availability when we employ macrodiversity. Figure 5.13 shows that the best performance can be achieved when both power control and macrodiversity are used. 


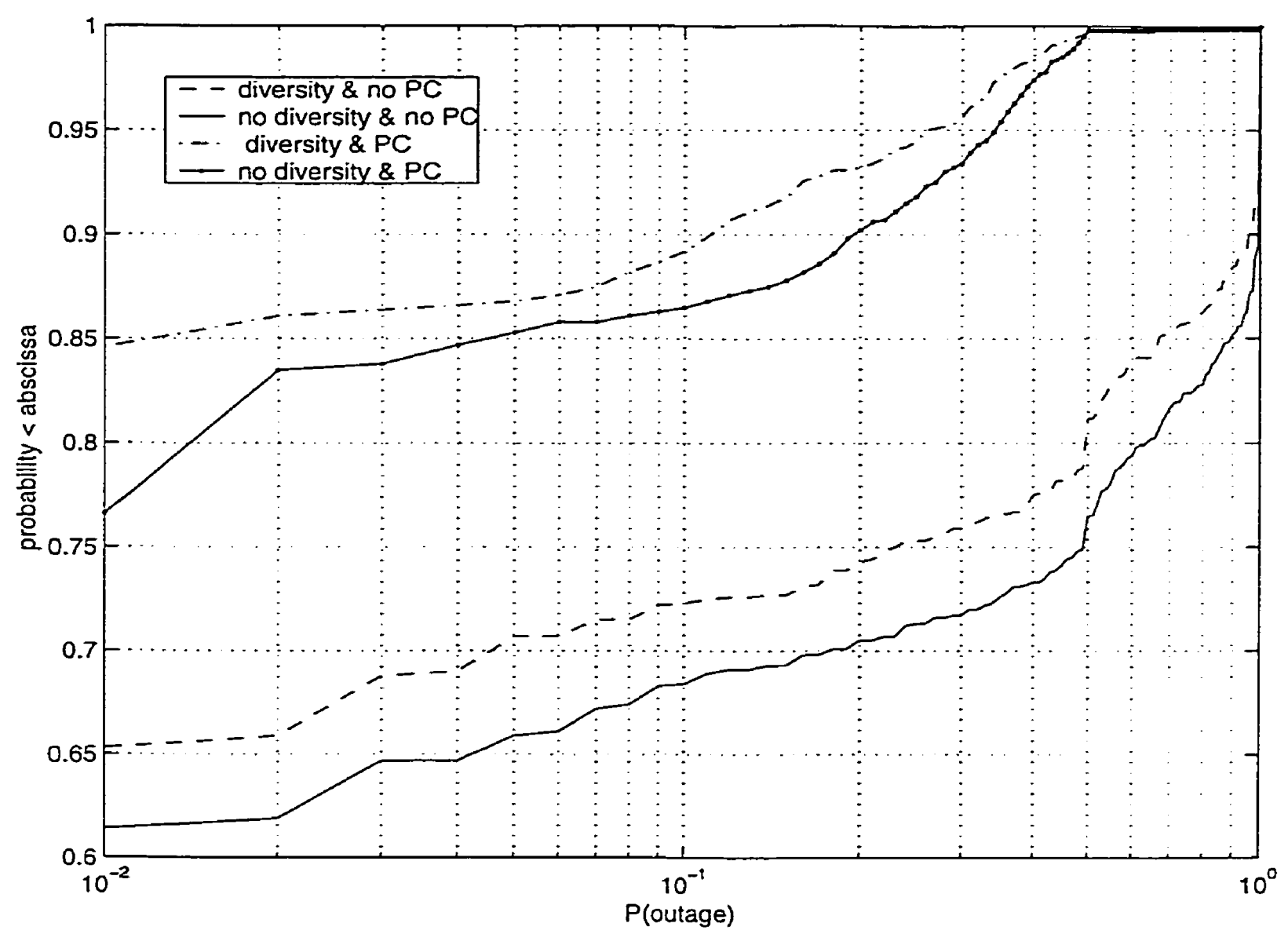

Figure 5.13 The effect of both power control and macrodiversity on system performance for $P C /$ snapshot $=30$, power control step $=2 \mathrm{~dB}$, and $\left(\mathbf{n}_{\mathrm{d}}, \mathbf{n}_{\mathrm{i}}\right)=(2,4)$.

\subsection{Autocorrelation function and power spectral density}

In this section, we will try to relate the power control per snapshot rate to the power control per 3-dB fading bandwidth. Power control updates are issued once per simulation sample, and the channel fading is unchanged during the number of samples in a snapshot.In order to be able to study the relation we are going to exploit the autocorrelation function of the Rician channel and the power spectral density function.

The time autocorrelation function for the Rician r.v $\beta$ is shown in Fig. 5.14. The power spectral density function of the fading signal is calculated from the Fourier transform of the autocorrelation function as shown in Fig. 5.15. The 3-dB fading bandwidth can be defined as the bandwidth required for the power spectrum to drop by $3 \mathrm{~dB}$ from the 
maximum value. It is shown in Fig. 5.15 that the $3 \mathrm{~dB}$ fading bandwidth is 10 (the units are inverse sample times). Then, for example if we were to use a PC/snapshot of 30 , the PC update rate would be $30 / 10=3$ times the $3 \mathrm{~dB}$ fading bandwidth.

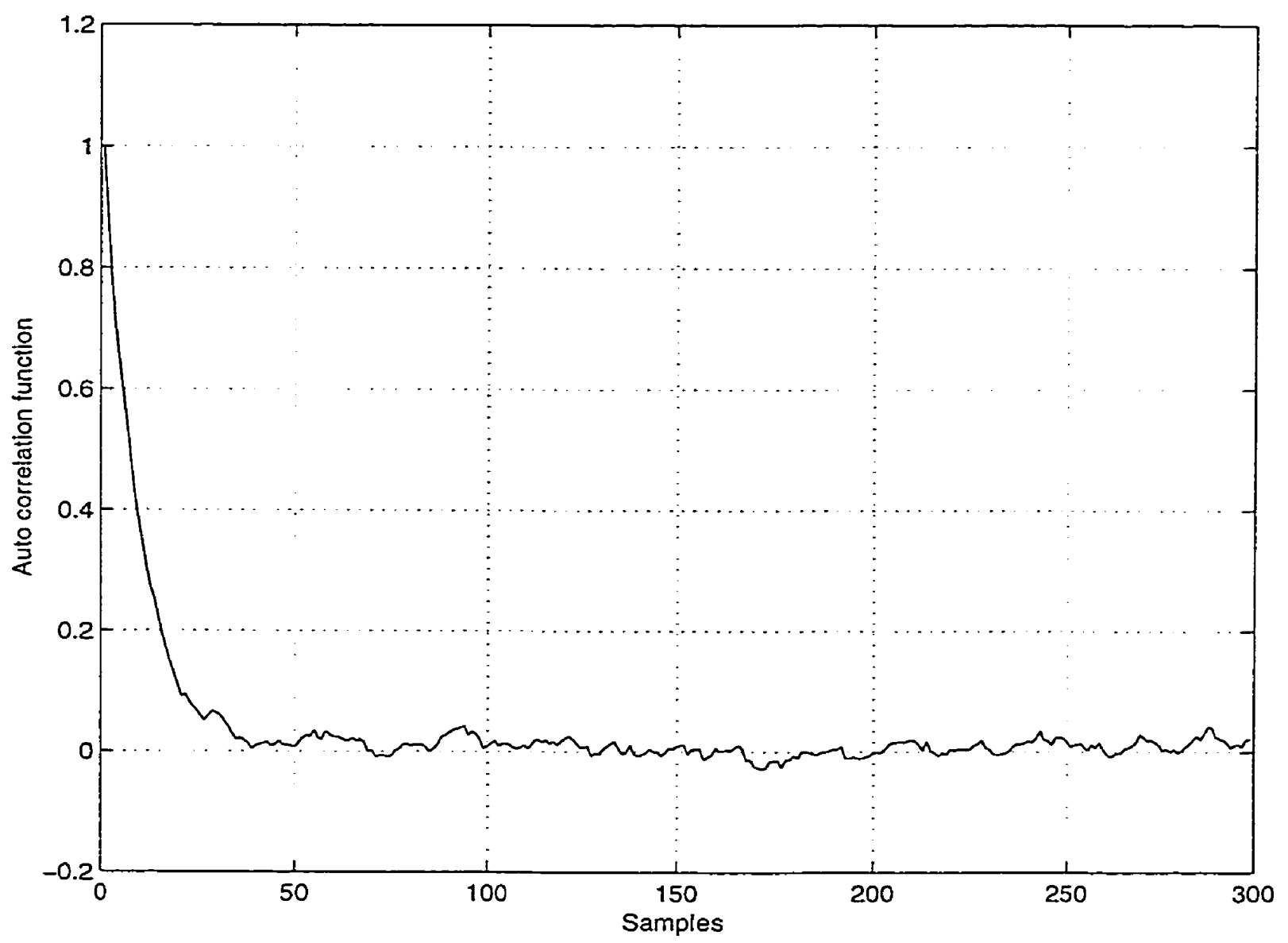

Figure 5.14 Autocorrelation function for Rician channel. 


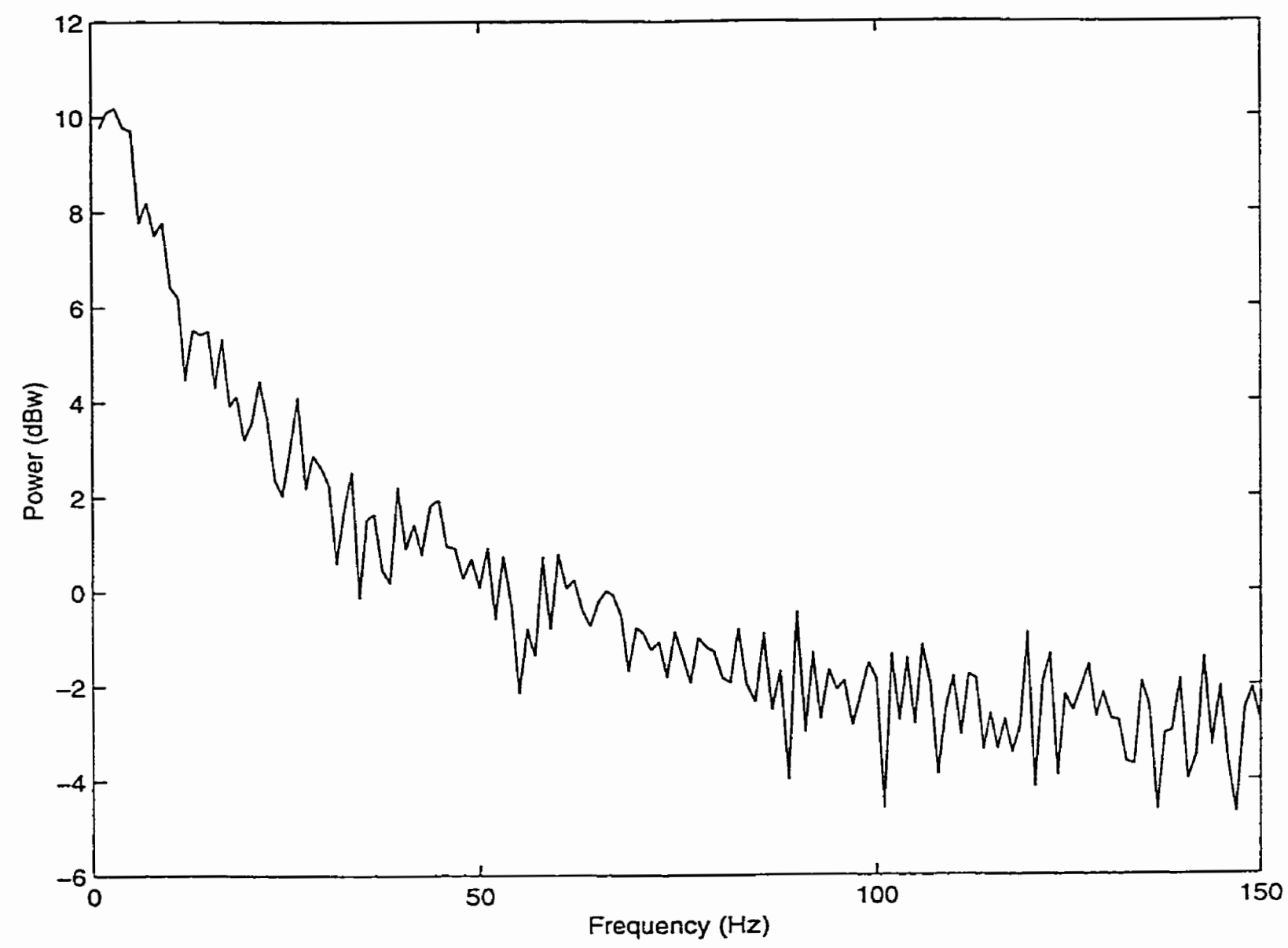

Figure 5.15 Power spectral density of the fading signal 


\section{Chapter 6 \\ CONCLUSIONS}

\subsection{Results Summary}

The simulation results in chapter 5 show that the propagation environment affects the LMCS/LMDS system availability. Transmitter power control and macrodiversity techniques have been implemented in this study. The LMCS/LMDS system availability is affected by the setting of the power control parameters such as the power control update rate, step size, transmitted dynamic range and the outer loop threshold. In this thesis, we also studied the effect of propagation parameters on system availability, some of these parameters such as path loss exponent, standard deviation of lognormal shadowing, Rician $\mathrm{K}$ factor and the correlation factor between fading samples.

The simulation results were shown in chapter 5 and can be summarized as follows:

The main focus of this study is to establish the optimum power control command update rate and the optimum power control step size. The simulation results in Fig. 5.1 shows that the binary SINR based power control with power control per snapshot of 100 and power control step of $0.5 \mathrm{~dB}$ gives the best system availability. However, if the $\mathrm{PC} /$ snapshot ratio is decreased to 50 we can achieve almost the same system availability but with a step size of $2 \mathrm{~dB}$. So, there is a trade-off relation between the power command rate and the optimum step size.

The transmitter dynamic range was also investigated in Fig. 5.2, we found that a constraint must be placed on the transmitted power. This constraint is essential to prevent the subscriber with the high outage probability from adding more interference to other subscribers in the system. Another important parameter is the power control outer loop threshold that depends on the required FER has been studied. This value can be changed 
according to the FER, if the FER is high we have to increase the outer loop threshold. Otherwise, the outer loop threshold should be lowered. Also, the modem threshold effect on system availability was shown in Fig 5.4.

For a fixed interferer propagation exponent of two, if the desired subscriber path loss exponent is increased from two to four the system availability will be degraded from 0.714 to 0.312 as can be shown in Fig. 5.5. On the other hand, referring to Fig 5.6 fixing the desired subscriber path loss exponent to two and increasing the interferer propagation exponent from 2 to 4 will improve the system availability. Therefore, the received interference will be attenuated more with a high path loss exponent.

The effect of the standard deviation of the log normal shadowing is also shown in Fig.5.7. We found that a higher value of standard deviation leads to more signal variations around the path loss distance, which causes more interference, and degrade the system availability.

The impact of a higher signal power on the LOS component of the desired subscriber signal, i.e. a higher value of Rician $\mathrm{K}$ factor will enhance the system availability as shown in Fig. 5.8.

If the fading channel is highly correlated in time the system availability is better than the case with low correlation. This can be explained as the power control update rate increased it will be able to track the multipath fading as shown in Fig 5.9.

Multistep SINR-based power control scheme depends on the power control mode, desired level (i.e. threshold), and the power control step size. These parameters have been investigated in this study. We found that power control with mode 1 will give the best system availability of 0.793. Also, we show that in Figure 5.10, a power control step size of $1 \mathrm{~dB}$ gives the best system availability for mode 1 . This value must be carefully chosen, since choosing too small or too large value will degrade the system availability. 
A comparison between two power control algorithms namely binary SINR based PC and multistep SINR-based PC is shown in Fig 5.12. The difference between the two schemes is small in terms of system availability. But the multistep power control scheme is transmitting more bits per commands. Therefore, the SINR based power control scheme is recommended.

Fig 5. 13 shows that employing macrodiversity has a significant effect on system availability for LMCS/LMDS system in the range of 5-10\%. Since the link between the subscriber and the base station is obstructed by buildings, trees, etc...

Macrodiversity helps to reduce the effect of these obstructions.

In our study, we define the system availability as the percentage of subscriber locations that have an outage probability of $1 \%$ or less. Also, we used a frequency reuse of 1 in each sector, which means there will be cochannel interference coming from all the sectors using the same polarization. In order to control the interference we can sacrifice some of the frequency band and forbidden some of the channels in each sector. This is referred to as the frequency reuse. Reducing the interference levels will enhance the received SINR at the base station and improves the outage probability.

\subsection{Future Research}

1. Study the effect of SINR balancing power control scheme on mitigating cochannel interference and enhancing the system coverage. This algorithm shows a promising improvement in minimizing the outage probability in mobile communications $[19,20$, 211.

2. Simulation study of adaptive modulation. Adaptive modulation has the advantage that it does not affect the interference. If the system is operating at maximum power level and required bit error rate is not achieved then a hybrid of adaptive modulation and power control scheme could be used. 
3. Simulation study for error control coding. It is well known that error control coding improves the performance by adding redundant bits in the transmitted bit stream that are used by the receiver to correct errors introduced by the channel, thus reducing the average bit error rate. This approach enables a reduction in the transmit power required to achieve a target bit error rate.

4. A study of the usage of repeaters to eliminate coverage holes and enhances the coverage. On the other hand, caution should be exercised and the interference that will be created by the repeaters should be investigated.

5. Rain fading is very critical problem in designing broadband wireless access systems operating at frequencies above $20 \mathrm{GHz}$. At such high frequencies the wavelength is in the order of $1 \mathrm{~cm}$ and even at low rain rates signal attenuation is very high, which has a severe effect on the area coverage.

6. The simulation is conducted based on the assumption of ideal polarization transmissions, which isolate the adjacent sectors completely. Actually, this is not practical and further investigation need to be conducted based on a more realistic assumption of polarization reuse. 


\section{REFERENCES}

[1] S. Ariyavisitakul, "SIR-Based Power Control in a CDMA System," IEEE GLOBECOM, Orlando, Vol. II, pp. 868-873, Dec. 1992.

[2] S. Ariyavisitakul and L. F. Chang, "Signal and interference statistics of a CDMA system with feedback power control," IEEE Transactions on Communications, Nov.1993, pp. 1626-1634.

[3] Chung-Ju Chang, Jeh-Ho Lee, and Fang-Ching. Ren, "Design of Power Control Mechanisms with PCM Realization for the Uplink of a DS-CDMA Cellular Mobile Radio System," IEEE Transactions on Vehicular Technology, Vol.45, No. 3, pp. 522529 August. 1996.

[4] Michele Zorzi, " Power Control and Diversity in Mobile Radio Cellular Systems in the Presence of Ricean Fading and Log-Normal Shadowing," IEEE Transactions on Vehicular Technology, Vol.45, No. 2, May 1996, pp. 373-382.

[5] Douglas A. Gary, "A Broadband Wireless Access System at 28 GHz," 1997 Wireless Communications Conference, Aug1 1-13,1997, pp.1-7.

[6] Scott Seidel and Hamilton W. Anold, " 28 GHz Local Multipoint Distribution Service (LMDS): Strengths and Challenges," Virginia Tech. Symposiums, June 1995.

[7] D. D.Falconer and G. Stamatelos, "LMCS system architecture and associated research issues," CITR Internal document, Dec. 9,1997.

[8] Scott Seidel and Hamilton W. Arnold, "Propagation Measurement at $28 \mathrm{GHz}$ to investigate the performance of LMDS", IEEE GLOBECOM, Nov 1995.

[9] Salina Q Gong, "Outage Performance with Directional Antennas in Cellular Fixed Broadband Wireless Access Systems", M. Eng. thesis, Carleton University, 1998.

[10] Ranjiv S. Saini, "Equalization Requirements and Solutions for Fixed Broadband Wireless Access Systems", M. Eng. thesis, Carleton University, 1998.

[11] Nausheen Naz and D. D. Falconer," "Temporal Variation Characterization for fixed wireless at $29.5 \mathrm{GHz}$ ", IEEE VTC 2000, Tokyo, May 2000

[12] J-P DeCruyenaere, "Propagation Simulation for the Prediction of LMCS/LMDS Coverage", ANTEM'98, OTTAWA, Aug 1998. 
[13] Peter B. Papazian et al, "Study of the Local Multipoint Distribution Service Radio Channel", IEEE Transactions on Broadcasting, Vol. 43, No. 2, June 1997, pp. 1-10.

[14] Vincentzio I. Roman, "Frequency Reuse and System Deployment in Local Multipoint Distribution Service", IEEE Personal Communications, Dec. 1999, pp. 2027.

[15] Douglas A. Gray, "Optimal Hub Deployment for 28 GHz LMDS System", Proc. 1997 Wireless Communications Conference, Aug.1997, pp.18-22.

[16] Donald C. Cox, "Cochannel interference considerations in frequency reuse small coverage-area radio systems", IEEE Transactions on Communications, Vol. Com-30, No.1, Jan. 1982.

[17] Michel D. Yacoub, Foundations of Mobile Radio Engineering. CRC Press, 1993.

[18] T.S. Rappaport, Wireless Communications, Principles and Practice, Prentice Hall, 1996.

[19] Jens Zander, "Performance of Optimum Transmitter Power Control in Cellular Radio Systems", IEEE Transactions on Vehicular Technology, Vol. 41, No. 1, Feb. 1992, pp.57-62.

[20] Jens Zander, "Distributed Cochannel Interference Control in Cellular Radio Systems", IEEE Transactions on Vehicular Technology, Vol. 41, No. 3, Aug. 1992, pp. 305-311.

[21] Sudheer A. Grandhi et al. , "Centralized Power Control in Cellular Radio Systems", IEEE Transactions on Vehicular Technology, Vol. 42, No. 4, Nov. 1993, pp.466-468.

[22] Hossein Alavi and Ray W. Nettleton, "Downstream Power Control for a Spread Spectrum Cellular Mobile Radio System”, Proc. IEEE GLOBECOM'82, 1982.

[23] J. M. Aein, "Power Balancing in Systems employing frequency reuse", COMSAT Technical Review, Vol. 3, No. 2, Fall 1973, pp.277-299.

[24] H. J. Meyerhoff, "Method for computing the Optimum Power Balance in Multibeam Satellite”, COMSAT Technical Review, Vol. 4, No. 1, Spring 1974.

[25] Bassam Hashem and Halim Yanikomeroglu, "CDMA Power Control Theory \& Implementation", IEEE Wireless Communications and Networking Conference 1999. 
[26] R. C. Bernhardt, "Macroscopic Diversity in Frequency Reuse Radio Systems", IEEE Journal on Selected Areas in Communications, Vol. SAC-5, No. 5, June. 1987, pp. 862-870.

[27] V. H. MacDonald, "The Cellular Concept", Bell Syst. Tech. J., Vol. 58, No. 1, Jan. 1979, pp. 15-41.

[28] G. M. Stamatelos and D. D. Falconer, "Millimeter radio access to multimedia service via LMDS", IEEE GLOBECOM'96, pp. 39-44, London U.K, Nov. 1996.

[29] K. S. Gilhousen, I. M. Jacobs, R. Padovani, A. J. Viterbi, L. A. Weaver, and C. E. Weatly, "On the Capacity of a Cellular CDMA System," IEEE Trans. Veh. Tech., Vol. VT-40, May 1991, pp.303-312.

[30] Bernard Caron et al. ,"LMCS Field Test in Canada ", ICPWC'99, pp. 124-129.

[31] Agne Nordbotten, "LMDS Systems and their Application", IEEE Personal Communications, June 2000, pp. 150-154.

[32] http:// www.ieee802.org/16. 